Florida International University FIU Digital Commons

$7-5-2013$

\title{
The Influence of Gender on Perceived Treatment Need among a Community Sample of Substance Users
}

Sheila P. Vakharia

Florida International University, svakh002@fiu.edu

DOI: $10.25148 /$ etd.FI13080716

Follow this and additional works at: https://digitalcommons.fiu.edu/etd

Part of the Social Work Commons

\section{Recommended Citation}

Vakharia, Sheila P., "The Influence of Gender on Perceived Treatment Need among a Community Sample of Substance Users" (2013). FIU Electronic Theses and Dissertations. 925.

https://digitalcommons.fiu.edu/etd/925 


\title{
FLORIDA INTERNATIONAL UNIVERSITY \\ Miami, Florida
}

\section{THE INFLUENCE OF GENDER ON PERCEIVED TREATMENT NEED AMONG A COMMUNITY SAMPLE OF SUBSTANCE USERS}

\author{
A dissertation submitted in partial fulfillment of \\ the requirements for the degree of \\ DOCTOR OF PHILOSOPHY \\ in \\ SOCIAL WELFARE \\ by
}

Sheila P. Vakharia 
To: Dean Michele Ciccazzo

R. Stempel College of Public Health and Social Work

This dissertation, written by Sheila P. Vakharia, and entitled The Influence of Gender on Perceived Treatment Need among a Community Sample of Substance Users, having been approved in respect to style and intellectual content, is referred to you for your judgment.

We have read this dissertation and recommend that it be approved.

Barbara Thomlison

$\begin{array}{r}\text { Frank Dillon } \\ \hline \text { Florence Keane } \\ \hline \text { Mark Macgowan, Major Professor }\end{array}$

Date of Defense: July 5, 2013

The dissertation of Sheila P. Vakharia is approved.

Dean Michele Ciccazzo R. Stempel College of Public Health and Social Work

Dean Lakshmi N. Reddi University Graduate School

Florida International University, 2013 


\section{DEDICATION}

I would like to dedicate this to my parents Prakash and Meena Vakharia.

You made me who I am and I would be lost without you.

I am grateful for your love and unwavering support.

Thank you. 


\section{ACKNOWLEDGMENTS}

This dissertation would not have been possible without the guidance and encouragement of my Chair, Dr. Mark Macgowan. I was so fortunate to have you as a mentor and I cannot express my gratitude to you. I would also like to thank Dr. Barbara Thomlison who was always available to me whenever I needed her support. Thanks to Dr. Frank Dillon for helping me to grow as both a researcher and statistician. I also appreciate the contributions of Dr. Florence Keane. In addition, I would like to extend my thanks to the doctoral faculty at the School of Social Work for being a valuable part of my education.

I would like to extend my thanks to the family, both near and far, who made it possible for me to pursue this degree. I love you all. Thanks to my sister Dimple, who supported my choice to move to Miami and who also made sure to visit me often! Love is a sister. Thanks for joining my family, Rajesh. Thank you to my extended family members on both the Vakharia side and the Thakker side. A special thanks to my Florida family- Vinod, Daksha, and Meehir Vakharia. It meant a lot to me to know you were here.

I am fortunate to say that I have had mentors all of my life. Many of them saw my potential long before I was able to see it in myself. Thank you to Laina Bay-Cheng for being my first social work mentor. You taught me so much. Thank you to Diane Wiener for being in my life. Words cannot express my admiration for you. Thank you to Nina Herzog for turning me into a harm reductionist. Thank you to Andrew Tatarsky for taking me under your wing and for believing in me. I am honored to call you a mentor. Thank 
you to Mark Sehl. You are a gentle and you are wise. Thank you to my Housing Works family. You changed me.

Thank you to my friends in New York who I have missed dearly during the past several years, but whose friendship and support also convinced me that I made the right decision to go back to school. Thanks, Becky. You're one of the greatest friends a girl could ask for. Thanks to both Alison and Luke for many fun times. You always believed in me, Lynan. Thank you to Gardiner, Andrew, Sonia, Lucia, and James. It was as though we were given a second chance at friendship because we missed out on each other at UR. I am proud to call you friends.

Thank you to the friends who entered my life in Miami and who provided me with support (and comedic relief) during the ups and downs of my doctoral education. Raquella, you never doubted me and were always by my side through this. I am ever grateful for having you come back into my life. Vanessa, you were my first friend here. Ramon, Megan, and Chris- you were my core group for the fun times and for the study times. I love you, Sherry. I couldn't have survived my first year here without you. Adi, thanks for being a great writing buddy. Thanks for being such a good friend, Julieta. Mariana and Bora- you were my inspiration. I respect and admire each of you.

Last, but not least, I would like to thank Mike. You have been with me since the beginning and you saw me through right to the end. Your love and support have been immeasurable during this period in my life and I couldn't have done it without you by my side. Thank you for your patience. Thank you for believing in me. Thank you for you. 


\title{
ABSTRACT OF THE DISSERTATION \\ THE INFLUENCE OF GENDER ON PERCEIVED TREATMENT NEED AMONG A COMMUNITY SAMPLE OF SUBSTANCE USERS
}

by

\author{
Sheila P. Vakharia
}

Florida International University, 2013

Miami, FL

\section{Professor Mark Macgowan, Major Professor}

Purpose: Most individuals do not perceive a need for substance use treatment despite meeting diagnostic criteria for substance use disorders and they are least likely to pursue treatment voluntarily. There are also those who perceive a need for treatment and yet do not pursue it. This study aimed to understand which factors increase the likelihood of perceiving a need for treatment for individuals who meet diagnostic criteria for substance use disorders in the hopes to better assist with more targeted efforts for genderspecific treatment recruitment and retention. Using Andersen and Newman's (1973/2005) model of individual determinants of healthcare utilization, the central hypothesis of the study was that gender moderates the relationship between substance use problem severity and perceived treatment need, so that women with increasing problems due to their use of substances are more likely than men to perceive a need for treatment. Additional predisposing and enabling factors from Andersen and Newman's (1973/2005) model were included in the study to understand their impact on perceived need.

Method: The study was a secondary data analysis of the 2010 National Survey on Drug Use and Health (NSDUH) using logistic regression. The weighted sample consisted 
of a total 20,077,235 American household residents (The unweighted sample was 5,484 participants). Results of the logistic regression were verified using Relogit software for rare events logistic regression due to the rare event of perceived treatment need (King \& Zeng, 2001a; 2001b).

Results: The moderating effect of female gender was not found. Conversely, men were significantly more likely than women to perceive a need for treatment as substance use problem severity increased. The study also found that a number of factors such as race, ethnicity, socioeconomic status, age, marital status, education, co-occurring mental health disorders, and prior treatment history differently impacted the likelihood of perceiving a need for treatment among men and women.

Conclusion: Perceived treatment need among individuals who meet criteria for substance use disorders is rare, but identifying factors associated with an increased likelihood of perceiving need for treatment can help the development of genderappropriate outreach and recruitment for social work treatment, and public health messages. 


\section{TABLE OF CONTENTS}

CHAPTER

PAGE

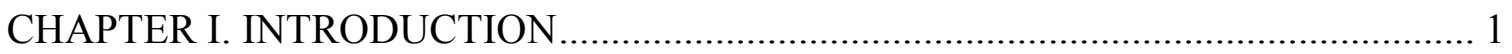

Substance User Perceived Treatment Need .............................................................. 3

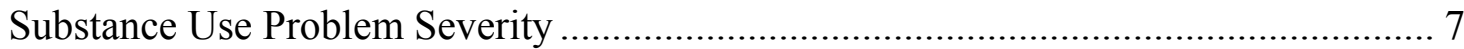

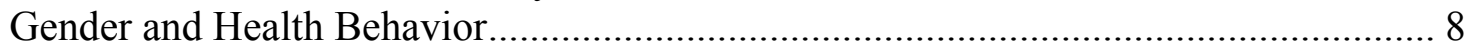

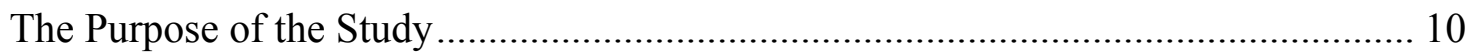

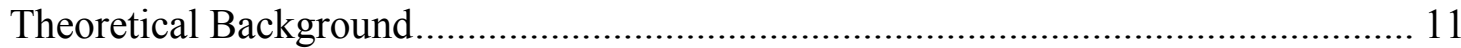

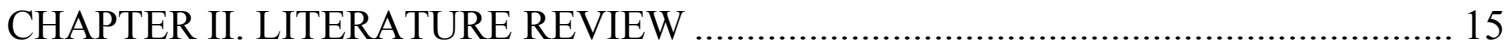

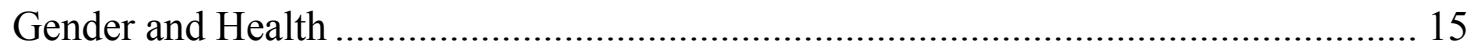

Gender and Substance Use in the United States ....................................................... 17

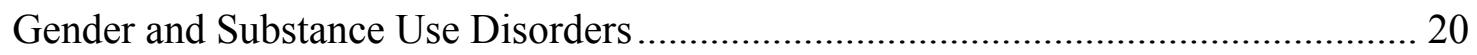

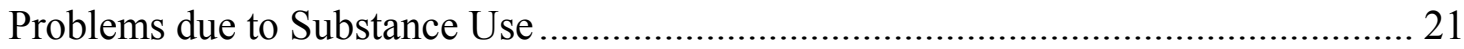

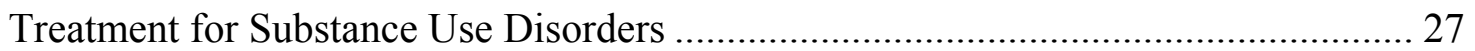

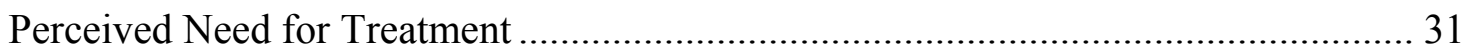

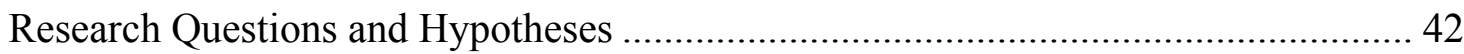

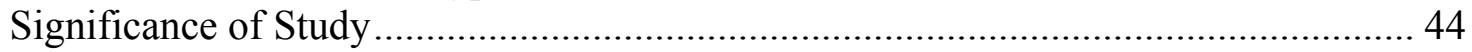

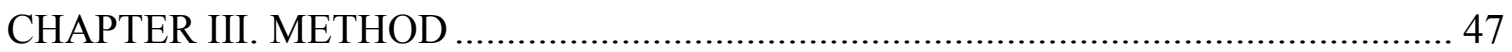

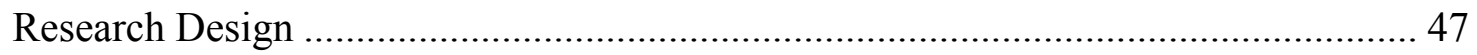

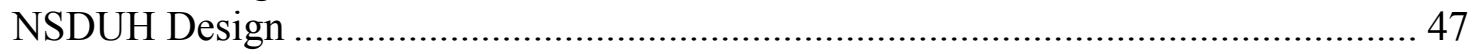

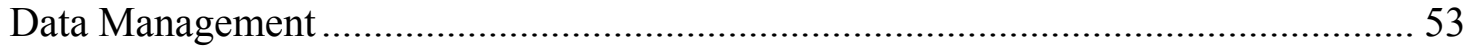

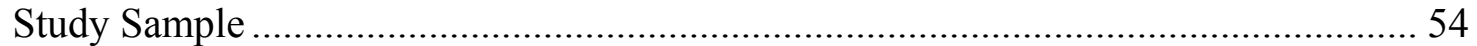

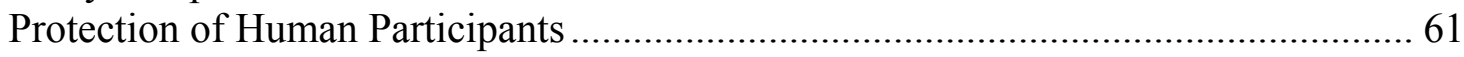

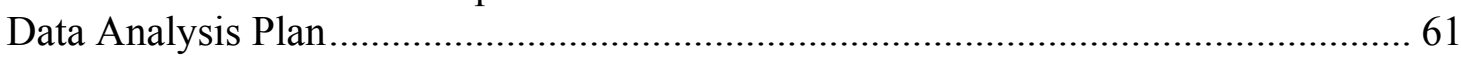

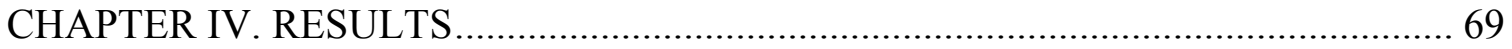

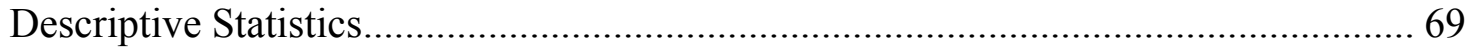

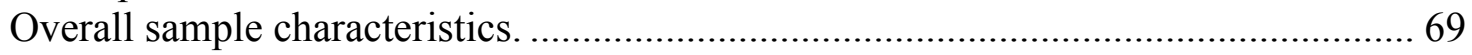

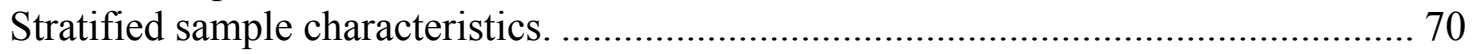

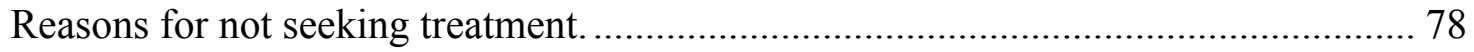

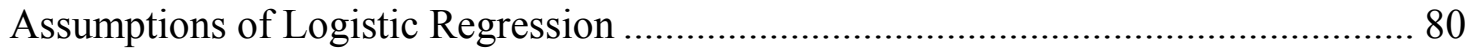

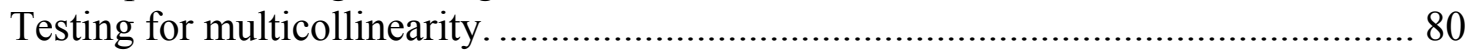

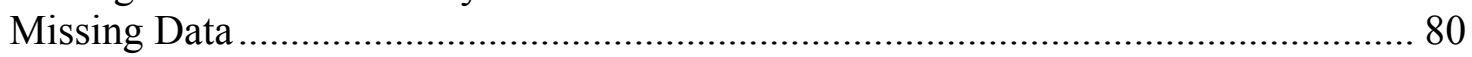

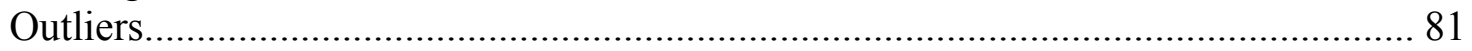

Logistic Regression and Rare Events Logistic Regression ........................................ 81

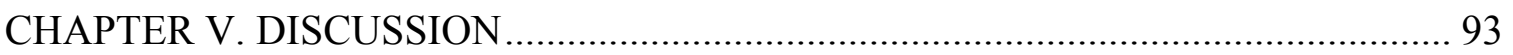

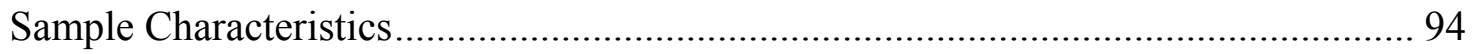

The rarity of perceived treatment need ...................................................................... 100

Reasons for not seeking treatment ....................................................................... 100

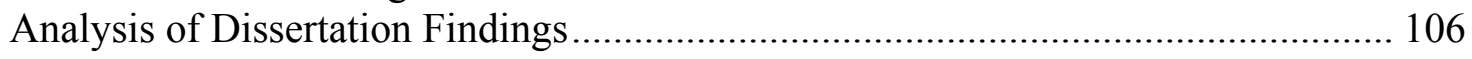


Substance use problem severity and perceived treatment need................................ 108

Effect of gender on perceived treatment need. .................................................... 110

Interaction effect upon perceived treatment need................................................... 111

Interaction effect while including predisposing and enabling factors. ...................... 112

Impact of predisposing and enabling factors on women ....................................... 118

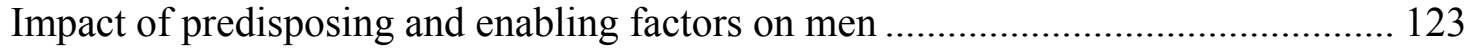

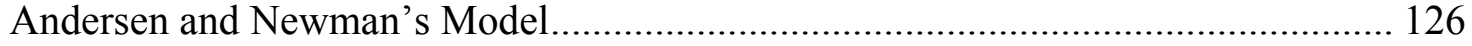

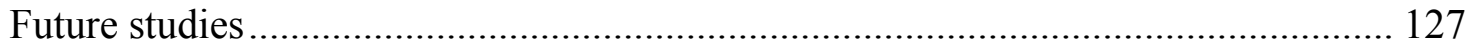

Limitations ...................................................................................................... 129

Implications for Social Work and Public Health ............................................... 131

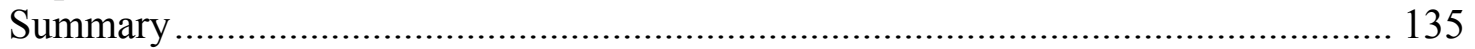

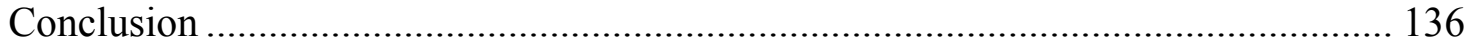

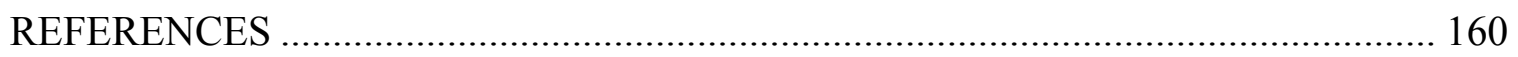

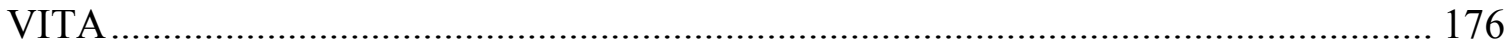




\section{LIST OF TABLES}

TABLE

PAGE

1. Descriptive Characteristics of Sample (Weighted and Unweighted).

2. Descriptive Characteristics of Sample, by Perceived Treatment Need and Gender.

3. Characteristics of Perceived Treatment Need Group by Gender.

4. Characteristics of No Perceived Need for Treatment Group by Gender

$144-145$

5. Most Commonly Reported Reasons for not Receiving Substance Use Treatment among Those who Perceived a Need for Treatment.

6. Most Commonly Reported Reasons for not Receiving Substance Use Treatment among Women who Perceived a Need for Treatment

7. Most Commonly Reported Reasons for not Receiving Substance Use Treatment among Men who Perceived a Need for Treatment. 148

8. Bivariate Correlations Between Variables.

9. Main Effect of Substance Use Problem Severity upon Perceived Treatment Need.

10. Main Effect of Gender upon Perceived Treatment Need.

11. Interaction Effect of Substance Use Problem Severity and Gender upon Perceived Treatment Need.

12. Interaction of Effect of Substance Use Problem Severity and Gender upon Perceived Treatment Need in Addition to Predisposing and Enabling Factors

13. Interaction of Effect of Substance Use Problem Severity upon Perceived Treatment Need in Addition to Predisposing and Enabling Factors for Women in Sample.

14. Interaction of Effect of Substance Use Problem Severity upon Perceived Treatment Need in Addition to Predisposing and Enabling Factors for Men in Sample..... 


\section{CHAPTER I. INTRODUCTION}

Few individuals who meet diagnostic criteria for substance use disorders (SUDs) actually pursue or receive specialty substance use treatment (Cohen, Feinn, Arias \& Kranzler, 2007; Wang et al., 2005a; 2005b). It is estimated that approximately 2.6 million Americans over the age of 12 received specialty substance use treatment in 2009, only a small fraction of those who met diagnostic criteria for SUDs that year (Substance Abuse and Mental Health Services Administration [SAMHSA], 2010b). According to SAMHSA (2010b), almost one in 10 (23.5 million) Americans over 12 years of age needed substance use treatment due to meeting diagnostic criteria for SUDs or engaging in risky substance use, and the vast majority (almost 90\%) of these individuals did not receive any treatment. This discrepancy has been described by experts (i.e. researchers, clinicians, and other health care providers) in the field of substance use as the "gap between need and service utilization" (Tucker \& Simpson, 2011, p. 371).

Expert estimates for substance use treatment need vary but, on both the epidemiological (macro) level and the clinical (micro) level, are commonly based upon whether individuals have met criteria for a SUD within the past year (Gfroerer, Penne, Pemberton \& Folsom, 2003; SAMHSA, 2010b; Tucker \& Simpson, 2011). Social indicators have also been used to indirectly estimate treatment need at a community level, including rates of substance-related mortality, morbidity, and criminal activity, so as to extrapolate potential treatment need (Stolzenberg, D’Alessio, Rivers \& Newell, 2003). Individual researchers and clinicians sometimes define treatment need differently as well, for example, based on current substance use after a reported history of substance use 
treatment (Rockett, Putnam, Jia, Chang \& Smith, 2005) or current heavy/risky substance use (Gfroerer \& Epstein, 1999; Tucker \& Simpson, 2011).

Certain characteristics have also been associated with greater expert determination of treatment need. Higher substance use problem severity (dependence rather than abuse) generally indicates a greater need for treatment (Druss et al., 2007; Ryan, Plant \& O’Malley, 1999), co-occurring mental health problems (Elhai \& Ford, 2002; Havassy, Alvidrez \& Mericle, 2009; RachBeisel, Dixon \& Gearon, 1999), and polysubstance use (Stewart, 2009) are also commonly determined to be indicators that an individual would be in need of treatment.

Yet there are those who caution against simply using diagnostic prevalence rates as a "proxy" (Narrow, Rae, Robins \& Regier, 2002, p. 115) for treatment need. This is due to three major reasons: (a) the limitations of diagnostic criteria themselves at identifying and distinguishing between substance use problems (Frances, 1998; Gillespie, Neale, Prescott, Aggen \& Kendler, 2007; Regier et al., 1998), (b) it is unclear whether rates accurately capture those individuals with "clinically significant" (Narrow et al., 2002, p. 115) impairment warranting intensive treatment rather than milder problems which may not (Spitzer, 1998), and (c) treatment need is determined objectively by experts rather than subjectively by substance users themselves so that "which definition [of treatment need] dominates depends on the constellation of political constituencies that have an interest in responding to the problem" (Weisner \& Schmidt, 2001, p. 110). These distinctions are important to make because if community-based prevalence rates are taken as a proxy for treatment need, they have many broad-reaching implications (e.g. scientific, clinical, economic, medical, policy among others) due to a seemingly 
"bottomless pit of possible demand" (Regier et al., 1998, p. 110) for psychiatric, mental health and/or substance use services which may benefit certain parties or negatively impact others.

\section{Substance User Perceived Treatment Need}

It was commonly believed that SUDs were uniquely distinguished from other disorders because individuals with these disorders were deemed incapable of acknowledging or recognizing the problems in their lives due to their substance use, let alone perceiving a need for treatment (Miller, 1983). Denial was (and, in some places still is) viewed as a distinct personality characteristic of individuals with substance use problems. But there is growing agreement and evidence to support the fact that many can and do, in fact, identify when their use is becoming problematic (Gerdner \& Holmberg, 2000; Groshkova, 2010; RachBeisel, Dixon \& Gearon, 1999; Ryan et al., 1995; Stewart, 2009).

It has been suggested that what is interpreted as denial among substance users is actually an interactive dynamic between two parties (often "expert" and substance user) where there is a disagreement about: (a) an understanding of the substance user's situation, (b) what the "problem" is (or whether there is one), and (c) the planned course of action (Miller, 1983). In these circumstances, due to the power differential between the expert and substance user, it is often presumed that the substance user's disagreement with the expert's opinion of any of these three factors is a manifestation of their altogether denial whereas it may be that their perception and understanding simply differs from that of the expert. It is also suggested that ambivalence about changing behavior can 
be mistaken for denial of the problem, when the substance user has not yet decided a course of action (Miller \& Rollnick, 2002).

If there are individuals who meet diagnostic criteria for SUDs in the community who do admit that they perceive a need for treatment, then it is likely these individuals (a) recognize that a problem exists, (b) do not believe that their problem can be addressed independently, and (c) believe that treatment is a valid means through which it can be addressed. An improved understanding of the association of gender with problem recognition and perceived treatment need among individuals who meet criteria for SUDs can better inform existing methods used for screening for substance use treatment need and making referrals for treatment. It is also important to understand the association of other demographic factors with the perceived need for treatment as well, such as race/ethnicity, age, health insurance status, etc. Those who perceive a need for treatment, but who have not pursued it, are an important group to target because a perceived need for treatment is a stage in the processes towards help-seeking (Mojtabai, Olfson \& Mechanic, 2002).

The perceived treatment need of substance users is also an important construct to the social work profession, which has a number of specific ethical responsibilities to clients (National Association of Social Workers [NASW], 1996). Social workers are responsible to remain primarily committed to the interests of their clients (only with the exception of circumstances when they are limited by the constraints of the law). In particular, social workers are distinguished from some other helping professionals with their ethical responsibility to encourage and support their clients' rights to selfdetermination. An individual's perceived need for treatment is essential to an effective 
and collaborative relationship. Additionally, social workers aim to empower their clients who may be otherwise accustomed to participating in relationships with providers who do not acknowledge the power differential between them. Social workers should respect that their clients are "experts" (p. 184) of their circumstances and work to collaborate with them respectfully during treatment (Freeman, 2001). In accordance with this, social workers should promote the rights of substance users to decide whether they want treatment and to work with them towards mutually agreed-upon goals. Better understanding the potential of gender to be associated with perceived treatment need among individuals who meet criteria for SUDs equips social workers to understand the perspectives of future clients.

The perception of treatment need also has theoretical significance in the field of substance use treatment and in understanding behavioral change. The Transtheoretical Model of Change (TTM) suggests that acknowledging client readiness for behavioral change is an important component of treatment and the helping process (Prochaska, Norcross \& DiClemente, 1995). According to TTM, there are five stages of change that people who change their behaviors generally pass through over time: precontemplation, contemplation, preparation, action, and maintenance (as well as relapse, at times). Each stage is characterized by certain tasks or behaviors which prepare the individual to move forward. There is no set period of time during which someone may stay in any of these stages and it can vary from person to person. The progression along stages can happen with or without formal treatment, in fact, this model was developed based on research collected on individuals who changed without treatment. Although no one can be forced to progress along the stages of change, people can be assisted in moving through stages 
by engaging them in various interpersonal processes which help them to clarify their goals and commit to behavior change. Individuals at precontemplation can be identified by the fact that they may be "unaware or underaware of their problems" (Norcross, Krebs, \& Prochaska, 2011, p. 144). The contemplation stage is characterized by an awareness that a problem exists yet no change-related action has been taken and no commitment has been made. Sometimes people in this stage are considered to be 'ambivalent' to changing; at once seeing both the pros to continuing the behavior but also seeing that there are pros to making behavioral changes. In the preparation stage, one has determined that a change needs to happen soon and options are being explored for how this problem can be resolved. The action stage is where the commitment to change has been solidified and the individual is engaging in lifestyle and behavioral changes directed at their goal, usually for at least the past six months (Norcross et al., 2011). Maintenance is when the person has maintained their changed behavior for long enough that they want to prevent a lapse into the former problematic behavior.

It could be said that the majority of participants in this study are probably in the precontemplative stage, as they meet diagnostic criteria for SUDs due to reporting substance-related problems yet do not perceive a need for treatment. The participants in the sample who reported perceiving a need for treatment are likely in the contemplation stage where they see that their substance use has become a problem that would probably benefit from treatment, however they have not committed to pursuing treatment for a number of reasons. The study's focus on individuals in these two stages of change is important, as they are individuals who could still be assisted in moving towards behavioral change to reduce the negative consequences of their substance use. 


\section{Substance Use Problem Severity}

As previously noted, one of the prominent factors associated with expert determined treatment need is the existence of substance use problems which meet diagnostic criteria for SUDs (Gfroerer et al., 2003; SAMHSA, 2010b; Tucker \& Simpson, 2011). Problematic substance use is distinguished from recreational substance use due to an individual's continued use of a substance despite experiencing predetermined number of substance-related negative consequences within the past year. A substance abuse diagnosis is the less severe SUD, while substance dependence is more severe due to experiencing a greater degree of "clinically significant impairment or distress" (p. 175) due to substance use (American Psychiatric Association [APA], 2000). There are also individuals who meet diagnostic criteria for SUDs for multiple substances at any given time, and they are most likely than other substance users to experience cooccurring psychiatric disorders and to be at greater risk for other health problems, including HIV transmission and overdose (Jones, Mogali \& Comer, 2012; Wu et al., 2011). In addition, individuals with both alcohol and illicit disorders generally comprise the majority of those in substance use treatment and survey data reveals that they have consisted of 42 to $46 \%$ of all clients in treatment from 2007 to 2011 (SAMHSA, 2012a).

Experts believe that treatment is necessary for individuals who meet criteria for SUDs because, unlike recreational users, it is presumed that they cannot stop using substances problematically on their own and require additional support and skillsbuilding to resolve the issue. However, expert determinations are not always aligned with the perceptions of those who are experiencing the problems themselves. It is worth exploring whether gender is associated with a perceived need for treatment among 
individuals who meet criteria for SUDs. This is a benefit of the study, in which the perceptions of individuals who meet diagnostic criteria for SUDs are the focus of exploration, given that they already meet expert-defined criteria for treatment need.

\section{Gender and Health Behavior}

It has been argued that "although many sociocultural factors are associated with health behavior, gender is among the most important" (Courtenay, 2000a, p. 4). Recent data on mortality in the United States reveal gender differences in life expectancy, with women living an average of five years longer than men (United States Department of Health and Human Services, 2011). Men are likely to engage in more health-related risk behaviors than women resulting in less healthy lifestyles which has been offered as a partial explanation for this (i.e., working in physically hazardous professions, heavy

alcohol use, cigarette smoking, less seat belt use) (Courtenay 2000a). Some of the gender differences between men and women are attributed to biological variations (such as the impact of testosterone on risk-taking and greater muscular strength making them more likely to choose physically demanding professions), although cultural expectations and social constructions of masculinity and femininity have also been theorized to impact gender-specific health-related beliefs and behaviors as well (Courtenay, 2000a; 2000b; Smith, Tran \& Thompson, 2008).

Men are less likely to perceive themselves as being at-risk for health problems and to over-estimate the quality of their current health than women, revealing different health beliefs than women (Courtenay, 2000a). Research on gender and help seeking suggests that there are also differences between the attitudes of men and women towards 
seeking professional help for their medical, mental health, and substance problems as well, often leading men to under-utilize those services (Courtenay, 2000b; Mansfield et al., 2005; Mansfield, Addis \& Mahalik, 2003; Neighbors \& Howard, 1987; Timko, Moos, Finney \& Connell, 2002). For example, despite comparable levels of lifetime psychiatric disorders among men and women, only "one in three persons seeking psychological care is a man" (Smith, Tran \& Thompson, 2008, p. 179). This underutilization of any type of health care leads to the situation where untreated problems can then be exacerbated or early detection for intervention or prevention cannot occur (Smith et al., 2008; Courtenay, 2000a).

The paradox of substance use treatment. The field of substance use is one of the few treatment settings in which men have historically been overrepresented (Green, Polen, Dickinson, Lynch \& Bennett, 2002). This has been attributed to several reasons: the greater incidence and prevalence of SUDs among men (SAMHSA, 2010b), the utilization of mental health services by women with co-occurring problems (Green et al., 2002; Greenfield et al., 2007), and the disproportionate impact of barriers which have prevented women from seeking substance use treatment (Green et al., 2002). Some of the barriers which have historically prevented women from seeking substance use treatment specifically include the stigma of being a substance-using woman (Brady \& Randall, 1999), childcare issues and family responsibilities (Green, 2006), lack of insurance coverage, transportation, and the fact that, up until recently, professional treatment was designed "by and for men" (Timko, Finney, \& Moos, 2005, p. 613). And although receiving mental health care for their needs is important, mental health facilities are not necessarily well-equipped to address the co-occurring substance use problems 
experienced by women. It has only been within the past decade that the number of women entering treatment has increased to start to become more proportionate with prevalence rates (SAMHSA, 2010a). Some believe that this is because the specific barriers which prevented women from pursuing treatment are being addressed, with some treatment programs providing accommodations for childcare and more gender-specific groups. As these barriers continue to decrease, it is hoped that regardless of gender, all individuals who perceive a need for treatment will be more willing and able to access it.

In light of previous findings about the influence of gender upon health and help seeking behaviors, it stands to reason that women may be more likely than men to interpret their substance use problems as requiring treatment yet they may not have been able to access that treatment due to barriers in the past and they may continue to experience some barriers today. In addition, not enough literature exists to explain how gender specifically affects the relationship between substance use problem severity and perceived treatment need among individuals who meet criteria for SUDs. Gaining insight into the role of how the gender affects their perceived treatment needs may provide a link toward better understanding how these individuals eventually seek professional help.

\section{The Purpose of the Study}

A number of studies have sought to identify the predictors of perceived treatment need among community samples of individuals who meet criteria for SUDs (Grella, Cochran, Greenwell \& Mays, 2011; Grella, Karno, Warda, Moore \& Niv, 2009; Mojtabai et al., 2002; Tucker, Wenzel, Golinelli, Zhou \& Green, 2011; Wu \& Ringwalt, 2004), however, none have explicitly examined the moderating influence of gender on the 
relationship between substance use problem severity and perceived treatment need despite the fact that it has been suggested that gender impacts health and help seeking (Courtenay, 2000a). The purpose of the proposed study is to understand how gender impacts the relationship between substance use problem severity and perceived treatment need among a community sample of individuals who meet criteria for SUDs. The hypothesized impact of gender is grounded in Andersen and Newman's model of individual determinants of health care utilization (1973/2005) - described in the next section. This conceptual model could explain why men are less likely to perceive a need for treatment than women

This investigation will utilize a cross-sectional design. The data source for the study will consist of secondary data from the National Survey of Drug Use and Health (NSDUH) on substance use, mental health, and treatment utilization of a community sample of household residents. The use of this large and extensive data set provides a unique opportunity to examine the relationship between the aforementioned variables.

\section{Theoretical Background}

The theoretical framework for health care utilization by Andersen and Newman (1973/2005) was the overarching guide for understanding which individual factors contribute to the perceived need for treatment although this study was not aimed attesting the theory itself. This theory will be summarized in this section, highlighting their model on the individual determinants of health service utilization which provided a rationale for the decision of which variables to include in the study (see Figure 1). Andersen and Newman's model can help to explain why women may be more likely than men to 
perceive a need for the treatment of their substance use problems, while also accounting for a number of other predisposing and enabling factors, which will be described below.

Individual determinants of health service utilization. The theoretical model developed by Andersen and Newman (1973/2005) has been used in several studies on perceived need for treatment (Falck, et al., 2007; Grella et al., 2009; Mojtabai et al., 2002), as well as those on mental health and substance use treatment utilization in general (Elhai \& Ford, 2002). It suggests that treatment utilization is influenced by a variety of factors, including individual predisposing factors (i.e. demographic characteristics, social structure, beliefs), enabling factors which allow for access to care (such as income, insurance coverage, community characteristics), and one's illness level (including the individual's own perception of illness, as well as the evaluation of illness by an expert). Individual predisposing factors are an important component of the model, because individuals with different demographic characteristics, social characteristics, and health beliefs utilize formal health care services at different rates. In particular, this model specifies age, gender, marital status and past illness as demographic individual predisposing factors which can predispose some individuals to utilize health services. Due to the nature of questions asked in the NSDUH, each of these could be included in the study. Social structure characteristics in the model include education, race, occupation, family size, ethnicity, religion, and residential mobility. Of these seven social structure characteristics, the only two which could not be included in the study were occupation and residential mobility. Among beliefs which may predispose individuals towards health service utilization are values around health and illness, attitudes towards 
health services, and knowledge about their illness. Unfortunately no questions regarding beliefs could be included in the study because they were not asked in the survey.

Predisposing factors also interplay with issues of access, or enabling factors, which can either contribute to increasing or decreasing the likelihood of using services. Enabling factors in the study's model include family factors such as income, health insurance, type of regular source of health care services, and access to regular source of health care services. The only two family-level enabling factors included were income and health insurance status. Enabling factors also include community factors including the ratio of health care facilities to population, the cost of health care services, the region of country, and whether the area is urban or rural. The only enabling community-level factor which could be included in the study was whether the area was urban, suburban, or rural. (This extra specification was made because the survey distinguished between three types of communities.)

According to this model, if an individual has predisposing characteristics which make them more inclined to use health care services in addition to enabling factors which can make services more accessible to them, they will be more likely to use services than those who do not. However, illness level is the third level which must also be considered. Illness level is comprised of two factors, the individual's perception of illness and an expert's evaluation of illness. The individual's perception of illness is theorized to consist of perceived disability, perceived symptoms, perceived diagnoses, and their perceived general state. In this study, the perceived need for treatment is used to represent the individual's perception of illness and it is believed that higher levels of problem severity may lead to an increased likelihood that participants will perceive their disability and 
symptoms. The evaluated illness is said to consist of expert-determined symptoms and diagnosis. In this study, all participants reported symptoms which met diagnostic criteria for SUDs from the DSM-IV-TR. All participants met the threshold of evaluated illness.

According to the model, if all three levels of individual determinants of health services utilization factors are aligned, it would theoretically make it more likely that this person will ultimately seek and access care. This model provided a rationale for the inclusion of a number of variables into the study which were already included in the survey as well. The hypothesized importance of gender and problem severity and perceived treatment need highlights gender as an individual level predisposing factor and problem severity as increasing the perception of illness among participants. 


\section{CHAPTER II. LITERATURE REVIEW}

\section{Gender and Health}

Health disparities can be defined as the "differences in health profiles across major subgroups of the population, including a broad spectrum of physical and mental health outcomes, from self-rated health to mortality, from psychological well-being to major mental disorders" (Schnittker \& McLeod, 2005, p. 75). These disparities exist between various segments in the U.S. population based on race, ethnicity, socioeconomic status, geographic region, gender, and other characteristics. They have increasingly become a target of much research due to the impact of disparate health on the lives of individuals, families, communities, and society as a whole.

In particular, there are notable differences in health status and life expectancy between men and women. According to reviews of health statistics, men have higher death rates for the top 15 causes of death in the U.S. (Courtenay, 2000b). A number of explanations have been offered to explain this, including biological, cultural, and sociological theories (Addis \& Mahalik, 2003). Although there are effects of testosterone, estrogen, and other biological factors which may lead to differential health outcomes, they cannot explain all differences between genders and their aggregate impact is relatively small (Courtenay, 2003). Cultural and sociological theories provide additional information about gender role socialization and cultural norms to suggest that health differences could also be the result of other processes (Mansfield et al., 2005). In particular, these lead to differences in health beliefs and health behaviors, such as helpseeking, which have been hypothesized to result in men having poorer health and higher 
mortality rates than women (Courtenay, 2003). The following section will highlight research on gender differences related to these factors.

Gender and health beliefs. There are a number of health beliefs whose impact upon health has been studied, including "self-rated health status, perceived susceptibility to risk, and readiness to change unhealthy behaviors" (Courtenay, 2003, p.5). Studies on these beliefs illustrate that, in general, men are more likely than women to (a) believe their health is better than it is by objective accounts, (b) to underestimate the risks of the harmful or dangerous behaviors they engage in, and (c) to be unwilling to change their current unhealthy behaviors (Courtenay, 2000a; 2000b; Courtenay et al., 2002; Mansfield et al., 2003; Mansfield et al., 2005). For example, a study of gender differences in health care utilization revealed a significant relationship between gender and self-reported health status, with women being more likely to report their health status and their mental health status as being poorer than men did (Bertakis, Azari, Helms, Callahan \& Robbins, 2000).

Gender and health behaviors. Men have less healthier lifestyles than women (Courtenay, 2000a), as illustrated by their greater involvement in risky behaviors that increase their likelihood of vehicular accidents, substance use problems, undetected and untreated health problems, sexually transmitted infections, and other chronic illnesses (Courtenay, 2000b). The direct and indirect relationship between these health beliefs and health behaviors, such as subsequent help-seeking, can be extrapolated, as individuals who are less likely to perceive illness would be less likely to seek medical attention. Men are less likely than women to have recently visited a physician or other health professional such as a dentist (Mansfield et al., 2003). Despite their comparable rates of 
mental health problems, they are also less likely to have sought the help of a mental health professional (Smith et al., 2008). Women are more likely than men to have recently visited a primary care provider and to have used diagnostic services (Bertakis et al., 2000).

\section{Gender and Substance Use in the United States}

The number of Americans who use mood-altering substances is steadily increasing (SAMHSA, 2010b). Men continue to use these substances at higher rates than women although this gap has been shrinking, especially among younger cohorts who are likely to hold more permissive attitudes towards both alcohol and illicit drug use than their predecessors and because women are less stigmatized for their use in current years than during generations past (Anthony \& Helzer, 1991; Green, 2006; Helzer, Burnam \& McEvoy, 1991; Kessler, 2005; Kessler et al., 1994; Robins \& Regier, 1991; SAMHSA, 2010b; Warner, Kessler, Hughes, Anthony \& Nelson, 1995). Whereas rates of alcohol consumption have stayed relatively stable in the past several years, both the use of illicit drugs and the nonmedical use of prescription medications are on the rise (SAMHSA, 2010b).

Alcohol use. Overall there are small, but notable differences between genders, so that men are more likely to report recent alcohol use than women (SAMHSA, 2010b). Men are also 30\% more likely to report engaging in binge drinking behavior than women, and are almost twice as likely to have recently engaged in several episodes of binge drinking within the past month as women. Biological explanations have been offered to explain the gender differences in binge drinking behavior due to physical and metabolic 
differences which lead women to experience intoxication after consuming less alcohol than men (Brady \& Randall, 1999; Collins \& McNair, 2002).

Altogether, the highest rates of alcohol use are currently among young adults aged 21 to 25 years, with an estimated $70.2 \%$ reporting recent alcohol use. Rates gradually decrease among older age groups, so that approximately $66.4 \%$ of adults between 26 and 29 years have recently used alcohol, and rates decline to $39.1 \%$ among individuals over age 65 (SAMHSA, 2010b). Younger cohorts of both men and women are more likely to meet diagnostic criteria for alcohol use disorders (abuse or dependence) than those who are older (Wu \& Ringwalt, 2004).

Differences between racial and ethnic groups are significant; with non-Hispanic whites being the most likely to report recent alcohol use than other racial and ethnic groups (SAMHSA, 2010b). Gender differences exist in various ethnic groups, generally, with women of a given racial/ethnic group consuming alcohol less frequently and less heavily than men in their racial/ethnic groups (Collins \& McNair, 2002).

Drug use. Fewer individuals report recent use of an illicit drug or the nonmedical use of prescription drugs compared to alcohol, though they still represent a significant portion of the population (SAMHSA, 2010b). According to current estimates, 22.2\% of young adults between the ages of 18 and 20 have recently used a drug and they are the age group with the highest rates of use. These rates also decrease with age; to roughly $20.5 \%$ for individuals between 21 and 25, 14.4\% among individuals between 26 and 29, and eventually dropping to $0.9 \%$ of individuals over age 65 .

The most commonly used illicit drug in the United States is currently marijuana (SAMHSA, 2010b). It is estimated that $6.6 \%$ of Americans over the age of 12 had used it 
within the past month and this number has been increasing over the years. Marijuana was followed by nonmedical use of prescription medications (e.g. pain relievers, tranquilizers, stimulants, and sedatives) by roughly $2.8 \%$ of individuals over age 12 . The nonmedical use of prescription medications is a relatively recent phenomenon documented in epidemiological studies, as questions related to it were not asked in the 1980s. Cocaine $(0.7 \%)$ and hallucinogens $(0.5 \%)$ were the third and fourth most commonly used illicit drugs among Americans.

The rate of drug use differs significantly by gender, with $10.8 \%$ of males over the age of 12 recently reporting use compared to $6.6 \%$ of females (SAMHSA, 2010b). This gender gap is closing with younger generations, as previous epidemiological studies reveal greater differences between genders (Swendsen et al., 2008). In particular, men are more likely than women to report the recent non-medical use of prescription medications, marijuana, and cocaine. Both men and women appear to have similar rates of recent nonmedical use of tranquilizers and methamphetamine (SAMHSA, 2010b). Recent research also suggests that women are more likely to be prescribed potentially addictive medications than men (Tetrault, Desai, Becker, Fiellin, Concato \& Sullivan, 2007).

Rates of recent drug use also differed significantly among racial and ethnic groups. American Indians and Alaskan natives reported the highest rates of recent drug use (18.3\%), followed by bi/multiracial individuals (14.3\%), black or African Americans (9.6\%), non-Hispanic whites (8.8\%), Hispanics (7.9\%), and Asians (3.7\%) (SAMHSA, 2010b). As with alcohol, women of ethnic/racial groups are less likely to have recently used a drug than men of their given ethnic/racial groups (National Institute on Drug Abuse, 1998). 


\section{Gender and Substance Use Disorders}

A review of several large-scale national epidemiological surveys reveals that the prevalence of SUDs has slightly increased over the past thirty years, with older epidemiological studies revealing lower past-year prevalence rates (Anthony \& Helzer, 1991; Helzer et al., 1991; Kessler, 2005; Kessler et al., 1994; Narrow, Rae, Robins \& Regier, 2002; Robins \& Regier, 1991; Warner et al., 1995). Several explanations have been given for this, including the changing diagnostic criteria for SUDs in various editions of the Diagnostic and Statistical Manual of Mental Disorders (DSM), the use of different survey methods, and generational cohort differences. However it should be noted that, despite the increased use of mood-altering substances in recent years, the percentage of Americans with clinically diagnosable SUDs (abuse and/or dependence) has remained relatively stable since 2002 (SAMHSA, 2010b).

Significant gender differences have been noted because current estimates suggest that twice as many men meet diagnostic criteria for SUDs than women (SAMHSA, 2010b). This gender differential has been demonstrated in decades of epidemiological research and the gap is closing among younger cohorts (Brady \& Randall, 1999). The most common SUDs between both genders are alcohol-related, followed by marijuana, and prescription medications (SAMHSA, 2010b).

Age has also been associated with SUDs because rates are lower among older cohorts. Young adults between the ages of 18 and 25 are most likely to meet criteria for SUDs compared to any other age group with prevalence rates at $20 \%$. Rates of SUDs also differ significantly between racial and ethnic groups. American Indians and Alaska Natives are the group with the highest prevalence rates $(15.5 \%)$, followed by 
bi/multiracial individuals (13.2\%). Rates were relatively comparable between Hispanics (10.1\%), non-Hispanic whites (9\%) and black or African Americans (8.8\%). In general, alcohol-related SUDs are most common, followed by drug-related SUDs, then cooccurring alcohol and drug SUDs.

In addition to the different prevalence rates between genders with regard to SUDs, they also experience their SUDs differently. The "telescoping effect" has been used to describe the experiences of women whose use of substances rapidly escalates to dependence. It "reflects a briefer time course for the development of medical consequences and behavioral/psychological factors characteristic of a dependence disorder" (Becker \& Hu, 2008, p. 37). Research suggests that women with SUDs have poorer familial and social resources than men and experience more problems in this facet of their lives than men (Brady \& Randall, 1999; Timko, Finney \& Moos, 2005). For example, women with alcohol-related SUDs are more likely to have a spouse who also engages in heavy drinking, which can impact attempts at cutting down drinking or receiving treatment.

\section{Problems due to Substance Use}

The use of mood-altering substances has been associated with a number of harms for individuals, families, communities and societies. Individual substance users themselves are at increased risk for a range of problems including substance-induced mental health disorders, physical health problems, relationship problems, overdose, and infectious diseases. These individual-level problems can eventually impact society due to 
costs associated with treatments, health care, criminality, and lost productivity (Babor et al., 2010; Davenport-Hines, 2001; DeGrandpre, 2006; Klein, 2008; Musto, 1973).

In an otherwise unprecedented attempt of its kind, researchers from Columbia University conducted a thorough review of local, state, and federal budgets in order to quantify some of the "costs" of substance use to society (The National Center on Addiction and Substance Abuse at Columbia University [CASA], 2009). There are, however, challenges associated with attempting to quantify the negative impact of substance use per se because it can be difficult to identify whether substance use was indeed the cause or a contributing factor to certain problems such as absenteeism. In this case, researchers maintained a conservative approach by focusing on more concrete and tangible sources of data so as not to risk overinflating the costs. They looked closely at the costs of mental health and substance use treatment, child welfare, public assistance, homelessness, adult corrections, juvenile justice, research, prevention, public safety, employment assistance and other types of programs which serve substance users or those affected by substance use.

On the federal level, they found the cost was approximately $\$ 238$ billion in 2005 alone, making it the sixth most costly area of spending, after "social security, national defense, income security, Medicare and other health programs including the federal share of Medicaid" (p. ii). According to CASA estimates, the costs associated with substance use and addiction for individual states ranked second only behind the costs of elementary and secondary education. When looking at the areas where most of the money was spent, they estimated that "almost three-quarters (71.1 percent) of total federal and state 
spending on the wreckage or burden of addiction is in two areas: health care and justice system costs" (p.iii).

Mental health. The use of substances can impact mental health by sometimes inducing other psychological disorders or exacerbating existing ones. The DSM-IV-TR lists nine substance-induced disorders, including sleep disturbances, mood disorders or even psychotic disorders (APA, 2000). Symptoms can range from mild mood changes or anxiety to more severe psychotic problems and many tend to subside after hours or days of abstinence although chronic long-term use of certain substances can lead to more lasting problems. For example, heavy ongoing use of amphetamines or methamphetamines is strongly associated with lifelong psychotic symptoms. Challenges associated with diagnosing substance-induced disorders include the difficulty distinguishing signs of intoxication or withdrawal from symptoms of a substance-induced psychological disorder, and also the high incidence of individuals who have pre-existing psychological disorders co-occurring with their SUDs (Torrens, Gilchrist, DomingoSalvany \& the psyChoBarcelona Group, 2011).

Gender. Research indicate that women with SUDs are more likely to experience co-occurring mood and anxiety disorders than men, while men are more likely to have been diagnosed with conduct disorders or antisocial behavior (Brady \& Randall, 1999; Kessler, Crum, Warner, Nelson, Schulenberg \& Anthony, 1997). Also, the presence of a pre-existing mental health diagnosis among women was more likely to indicate that they would later meet diagnostic criteria for an alcohol-related disorder than for men (Kessler et al., 1997). Women are also more likely to experience trauma and victimization both prior to their SUDs and as a result of their SUDs than men (Brady \& Randall, 1999). 
Physical health. Substance use has been associated with various physical health problems and infectious disease transmission (Babor et al., 2010). People under the influence of mood-altering substances are at higher risk for unintentional accidents and injuries because many substances can impair judgment, reaction time, cognitive processing, and physical coordination. Impaired judgment can also contribute to other forms of risky behavior such as unsafe sex, which can result in unplanned pregnancy and sexually transmitted infections. Driving while impaired by alcohol and/or illicit drugs is against the law but it is still a common occurrence. In 2009, 30.2 million Americans admitted to driving under the influence of alcohol in the past year and 10.5 million admitted they drove under the influence of an illicit drug (SAMHSA, 2010b). The Centers for Disease Control and Prevention (CDC) estimates that alcohol is involved in almost one third of all traffic-related deaths and illicit drugs are involved in $18 \%$ of all driver deaths (Center for Disease Control and Prevention, 2011).

The type of substance used can increase the likelihood of certain health problems. Stimulants (cocaine, amphetamine, methamphetamine, and other stimulants) activate the central nervous system, increase heart rate, suppress appetite, and raise blood pressure. Their use is associated with sleep disturbances, weight loss, malnutrition, dehydration, irregular heartbeat and other heart problems, increased body temperature (which can lead to organ damage), and risk of stroke or heart attack (Williamson, Gossop, Powis, Griffiths, Fountain \& Strang, 1997). A recently published study on a sample of long-term cocaine users with no apparent cardiac symptoms and regular heartbeats still found a number of signs of "silent myocardial damage" (Aquaro et al., 2011). 
Route of administration, or the method in which the substance is ingested, also predicts risk for potential health problems and infectious diseases. Ongoing use of substances that are smoked, such as marijuana, crack cocaine and methamphetamine, are likely to contribute to respiratory problems and cancers because the lungs are directly exposed to the drug itself and other chemical additives it may contain (Restrepo, Carrillo, Martinez, Ojeda, Rivera \& Hatta, 2007). Continued snorting of drugs such as cocaine or heroin increases the likelihood of damage to the mucous membranes of the nasal cavity and can lead to other sinus problems. Injecting drugs comes with the risk of abscesses, vascular damage and infections. It is estimated that intravenous drug use has contributed to over one third of cases of HIV in the United States since the epidemic began (CDC, 2002). Hepatitis $C$ is currently the number one blood-borne communicable disease in America and it is estimated that over $75 \%$ of all injection drug users have been infected with it (National Institute on Drug Abuse, 2000). According to the CASA report, problematic substance use (both licit and illicit) "alone causes or contributes to more than 70 other diseases requiring hospitalization" (2009).

Gender. Gender differences in the medical consequences of substance use have also been recognized. Female substance users are at greater risk than men for certain health and medical problems resulting from their substance use. For example, liver disease is more severe among female long-term drinkers and they are also more likely to experience effects upon their reproductive organs (Brady \& Randall, 1999). In addition, women with SUDs are more likely to be at risk for infectious and sexually transmitted diseases (such as HIV, Hepatitis B, and Hepatitis C) (Miller \& Neaigus, 2001). It has been suggested that this is because they are more likely to have partners who are also 
substance users, and they perceive greater barriers to safer sex practices. In particular, women who inject drugs are often initiated into injection by their sexual partners who are longer-term users who may already have such diseases (Miller \& Neaigus, 2001). Women who continue to use substances during pregnancy also increase the risk of complications during delivery and subsequent problems in the fetus. These differing effects of substance use on the health of women illustrate the importance of ensuring women can access substance use treatment.

Justice. In addition to contributing to large societal costs due to health care, substance use also creates costs associated with the criminal justice system. The manufacture, distribution, use and/or possession of illicit drugs is against federal law and is considered a "drug-defined offense" (Bureau of Justice Statistics, 2010). There were roughly 1,663,582 arrests for drug-defined offenses in the United States in 2009 (Federal Bureau of Investigation Uniform Crime Report, 2010). The vast majority of these arrests $(81.6 \%$ or $1,357,483)$ were for simple possession charges and the remaining charges were for the sale or manufacture of drugs. Drug-defined offenses accounted for $12 \%$ of total arrests $(13,687,241)$ in the United States that year. The cost of staffing police forces, booking offenders, conducting trials, and incarceration are all related to these arrests.

Substance use has also been associated with violence and criminality since the late $19^{\text {th }}$ century in America (Musto, 1973). Goldstein's (1985) conceptual model linked illicit drug use to violence in three specific ways (a) psychopharmacological violence susceptibility to being the perpetrator (or victim) of violence due to being under the influence of drugs (b) economic compulsive violence - violent crimes conducted to gain 
the means to purchase drugs and (c) systemic violence - the traditionally aggressive patterns of interaction within the system of drug distribution and use" (p. 497).

It has been argued that disproportionate amounts of money are being spent upon "shoveling up the wreckage" (p. 2) of substance use problems compared to expenditures on other programs and approaches which could prevent such tremendous costs to individuals and society (CASA, 2009). Substance use treatment is seen as an efficient and cost-effective investment which can both contain and reduce the impact of substance use. The proposed study's focus on perceived treatment need is meant to contribute to the knowledge on the factors that may contribute to a perceived treatment need among community-based substance users who could benefit from such services.

\section{Treatment for Substance Use Disorders}

The number of specialty substance use treatment facilities in the United States has remained relatively stable in the past several years, with an estimated 15,390 functioning facilities as of 2010 (SAMHSA, 2011). Specialty substance use treatment facilities include those which provide various levels and intensity of care, such as: detoxification, inpatient rehabilitation, outpatient rehabilitation and therapy, and therapeutic communities. Psychosocial and medical interventions are now the most predominant forms of specialized formal treatment provided to address SUDs in these settings.

Formalized diagnostic criteria have helped to lead to the expansion of both a professionalized treatment and pharmaceutical industry for SUDs for over half a century now, replacing the inebriate houses and various other questionable "treatments," which

were common until the 1940s (White, 1998). "Calling alcoholism a disease ... is a useful 
device both to persuade the alcoholic to admit his alcoholism and to provide a ticket for admission into the health care system" (Vaillant, 1995, p.22). Substance use treatment, like other medical treatments, is covered by most insurance companies under the label of behavioral health care (Weisner \& Schmidt, 2001).

Substance use treatment has been generally associated with positive outcomes for participants, leading to reduced substance use or complete abstinence, reduced high-risk behaviors, and other benefits for many individuals (Wild et al., 2002). However, most of these benefits can only occur after some degree of engagement in it. Research shows that attendance, compliance and completion are distinct challenges in substance use treatment settings (Najavits, Crits-Christoph \& Dierberger, 2000; Weisner, Mertens, Tam \& Moore, 2001). Not everyone who experiences substance-related problems pursues treatment, receives it, or engages in it fully.

The first weeks of substance use treatment are especially critical points of engagement as it is estimated that approximately $15-28 \%$ of individuals do not return for the second session of treatment (Coulson, Ng, Geertsema, Dodd \& Berk, 2009) and 30\% drop out within the first month (Palmer, Murphy, Piselli \& Ball, 2009). In addition, a national survey revealed that only $41 \%$ of all clients completed treatment, with the remainder of clients being discharged due to drop out, incarceration, or referral for further care (SAMHSA, 2008). It is also common for individuals who complete treatment to experience some degree of backsliding in their substance use within the first year after they have completed treatment (Witkiewitz \& Marlatt, 2007). A number of factors have been attributed to these problems in substance use treatment (Coulson et al., 2009; Marlatt \& Witkiewitz, 2010; Tatarsky, 2003). One could generally be categorized as 
"motivational problems" (Ball, Carroll, Channing-Ball \& Rounsaville, 2006; Ryan et al., 1995; SAMHSA, 2010b; Weisner et al., 2001). Such problems can include: varying degrees of importance placed upon treatment for one's substance use problems (Weisner et al., 2001), believing that one could resolve their problems on their own (Ball et al., 2006; SAMHSA, 2010b), and not being intrinsically motivated for treatment at the time (Ryan et al., 1995).

One way that individuals end up in treatment (whether or not they perceive a need for treatment) is due to the influence of outside social pressures and controls (Polcin \& Weisner, 1999). It has been estimated that up to $75 \%$ of individuals who enter substance use treatment in the United States do so as a result of at least one social control tactic (Wild, 2006). Three main types of social controls have been identified as influencing decisions to enter treatment: legal social controls related to the criminal justice system such as court-mandated treatments or diversion programs, formal social controls through other institutions such as employee assistance programs or social services, and informal social controls from interpersonal relationships with individuals such as a spouse, family member or friend (Wild, 2006). These tactics can be quite effective at getting individuals into treatment but evidence about their impact upon treatment engagement, retention and outcomes is mixed (Farabee, Shen \& Sanchez, 2002; Gregoire \& Burke, 2004; Wild, Roberts \& Cooper, 2002). There are also legal and ethical debates about the merits of the use of legal social controls so as to get otherwise unwilling individuals into treatment. Research does suggest that if individuals themselves do believe that they need treatment (regardless of social controls) and are motivated to participate in treatment then they are 
more likely to engage in it and achieve goals (Wild, Cunningham \& Ryan, 2006; Zeldman, Ryan \& Fiscella, 2004).

Gender and substance use treatment utilization. Men experience SUDs at higher rates than women and their rates of enrollment in substance use treatment historically have been even higher than those of women. This is due to a combination of different sociocultural factors, as well as economic and institutional barriers that prevented women from seeking substance use treatment (Brady \& Randall, 1999; Green, 2006; Greenfield et al., 2007; SAMHSA, 2010a; Weisner, 1993). It is unclear if, despite their underutilization of substance use treatment services, women were (or have been) less likely to perceive a need for substance use treatment. Given the literature on the increased likelihood of problem recognition and health care utilization, it is possible that they have greater perceived need for treatment but could not pursue it in the past.

Sociocultural barriers. Research on cultural constructions of the meaning of alcohol or drug use differentially impact men and women (Collins \& McNair, 2002). Women are often held to different standards than men regarding substance use and the fulfillment of family responsibilities so that they have been more harshly judged and stigmatized when they have experienced problems due to their substance use (Brady \& Randall, 1999). For example, many Latino subcultures find alcohol use to be acceptable among men, but not among women (Collins \& McNair, 2002). This was also true here in the United States, but there is some evidence that attitudes towards alcohol and drug use in recent generations are more similar between genders and the prevalence of SUDs among women are more acknowledged (Green, 2006). After immigrating to the United States, more acculturated Latina immigrants have been found to engage in alcohol use 
than would have otherwise while in their countries of origin because of more permissive attitudes here (Collins \&McNair, 2002). Women of many other ethnic and racial groups have shared similar stigmas surrounding their use, leading a number to be reluctant to pursue treatment for fear of the stigma of being recognized as a substance user (Green, 2006).

Economic and institutional barriers. In addition, accessibility to treatment has been a barrier to substance-using women (Greenfield, et al., 2007). For example, women with children are more likely to have problems committing to treatment due to childcare responsibilities. In addition, women generally have lower incomes and educational attainment than men, making it difficult for them to afford the costs associated with treatment if they lack health insurance.

Outcomes. Despite differences in utilization, recent research on gender and substance use treatment outcomes is promising and suggests that women are just as likely as men to have positive outcomes as a result of treatment (Green, 2006; Green et al., 2002; Greenfield et al., 2007). In their study on predictors of different treatment processes, Green and colleagues (2002) did not see any gender differences for treatment initiation, completion, or time spent in treatment. Greenfield and colleagues (2007) also suggest that once in treatment, they did not see evidence for gender differences in treatment retention, completion, or outcome.

\section{Perceived Need for Treatment}

Perceived or felt treatment need has increasingly been discussed in both the substance use and mental health literature because of the growing use of social controls to 
get many individuals into treatment and questions about whether individuals actually believe they need the treatment they are receiving. Studies aim to better understand the perceptions and experiences of the individuals themselves who may be mandated or coerced into treatment regardless of their own perceived need for treatment. Basically, these studies aim to primarily determine the individual-level predisposing factors (correlates of perceived need or those factors which are associated with perceived need) that can contribute to eventual help-seeking and service utilization (Andersen \& Newman, 1973/2005). There are studies which focus solely on the factors leading to perceived treatment need for mental health disorders such as depression or anxiety (Farabee et al., 2002; van Beljouw et al., 2010), for co-occurring mental health and SUDs (Grella et al., 2011), and a body of others for SUDs alone (Grella et al., 2009; Mojtabai et al., 2002; Ryan et al., 1995; Tucker et al., 2011; Wu \& Ringwalt, 2004).

Problem severity. Those who meet criteria for the more severe SUD, substance dependence, experience greater problems due to their substance use than individuals who meet criteria for substance abuse and are deemed to require more frequent and intense treatment according to expert determinations of treatment need (Gfroerer \& Epstein, 1999; Grella et al., 2009; Rockett, Putnam, Jia \& Smith, 2003; Weisner \& Schmidt, 2001). Studies on related constructs such as help-seeking (Tucker, 1995) and internal motivation during treatment (Ryan et al., 1995) suggest that greater problem severity increases the likelihood of both. Yet studies on perceived need have not accurately determined whether problem severity also dictates substance users' perceived need for treatment, although it stands to reason that they would be related. 
There are those studies on perceived need for treatment which lump together all individuals who meet abuse or dependence criteria into one group in their sample, blurring any potential differences in perceived need between groups who actually have varying degrees of substance-related problems and SUDs (Edlund, Unutzer, \& Curran, 2006; Elhai \& Ford, 2002; Mojtabai et al., 2002). There are those which only focus on individuals meeting criteria for substance dependence without the comparison to individuals with other degrees of substance use problems such as abuse or having multiple SUDs (Grella et al., 2009; Wu \& Ringwalt, 2004). In particular, Wu and Ringwalt's study (2004) on perceived treatment need was based on a sample of community-based women who only met criteria for alcohol dependence. Falck and colleagues' study (2007) on perceived treatment need focused only on rural illicit stimulant drug users and measured problem severity with the Addiction Severity Index instead of the DSM which is the general standard for diagnosis in the U.S. In Grella and colleagues' study (2009), it was found that over $75 \%$ of individuals who perceived a need for treatment experienced two or more substance-related problems in the past year in addition to already meeting substance dependence criteria (Grella et al., 2009). However, it is unclear what types of problems the researchers were referring to. Edlund and colleagues (2006) also reported on perceived treatment need for SUDs, however lumped together alcohol or drug disorders without specifying whether they were abuse or dependence diagnoses. In addition, they did note whether people had up to three diagnoses (and it lead to increased perceived need for treatment), but they did not distinguish between substance use and/or other mental health problems. 
Only one study in this area has explored the possible impact of multiple SUDs upon the perceived need for treatment and it was a study of community based women with alcohol dependence (Wu \& Ringwalt, 2004). It was found that women who met criteria for dependence for both alcohol and an illicit drug were seven times more likely to perceive a need for treatment than women with alcohol dependence alone. There are also studies which examined specific substances, making it unclear whether participants have used anything else (Falck et al., 2007). This is an area worth exploring, as individuals who use more substances may think they could benefit from professional help. Polysubstance use is relatively common, with an estimated $9.7 \%$ of the US population over the age of 12 reporting that they have used alcohol in conjunction with illicit drugs (SAMHSA, 2010b). Those who meet diagnostic criteria for multiple substances may have different experiences than those who meet criteria for a single substance.

More research in this area will be informative and could clarify the relationship between polysubstance use disorders and perceived treatment need. The proposed study aims to fill this gap by including individuals with three different degrees of substance use (monosubstance abuse, monosubstance dependence, and multiple SUDs) in its sample so as to tease apart how these levels impact a substance user's perceived need for treatment.

Gender. Research suggests that gender is potentially related to perceived need for treatment, with women being equally (Grella et al., 2009) or more likely (Edlund et al., 2006; Grella et al., 2011; Mojtabai et al., 2002) to believe they need substance use treatment than men. Yet more research is needed because this discrepancy could be the result of different sampling and methodological issues in these studies. 
Grella and colleagues (2009) conducted a secondary data analysis of the National Epidemiologic Survey on Alcohol and Related Conditions (NESARC) with a community-based national probability sample survey in which they compared three subsamples of individuals who met criteria for substance dependence: those who received mental health or substance use treatment in the past year $(n=236)$, those who perceived a need for treatment but did not receive it $(\mathrm{n}=150)$, and those who neither perceived a need for treatment nor receive it in the past year $(n=1,216)$. They found no significant differences in gender between any of the subsamples. However it should be noted that the "perceived need" group was the smallest of the three, consisting of only 92 men and 58 women in the analysis. With such a relatively small group, it may be that differences could not have been detected. Also, the sample for analysis was limited to individuals who only met criteria for substance dependence, excluding those with different levels of problem severity such as those who met criteria for a substance abuse diagnosis. It is unclear whether the levels of problems due to their substance use may have been related to the perception of treatment need.

Gender and sexual minority status were found to have a significant relationship with perceived treatment need in a secondary data analysis of a survey with a community-based single state probability sample (Grella et al., 2011). Among those who did not receive treatment during the prior year, women with mental disorders (mood, anxiety and/or substance use), regardless of sexual orientation, were more likely than men to perceive a need for treatment. In this analysis, the "perceived need" group was larger $(\mathrm{n}=516)$ and consisted of 315 women and 201 men. Yet the findings have limited external validity due to the fact that it lumped together all mental disorders, without 
studying the potentially distinct characteristics of individuals with SUDs. Also, the sample is not nationally representative because it was comprised of residents of one single state (California) with potentially unique demographic characteristics.

Mojtabai and colleagues (2009) analyzed data taken from the National Comorbidity Survey (NCS) where they reviewed rates of perceived need for treatment among individuals who met criteria for SUDs, mood disorders, and anxiety disorders. They found that women were more likely than men to perceive a need for treatment for any of the three disorders. However, the survey based the diagnosis of disorders upon the DSM-III-R which had different diagnostic criteria than today's DSM-IV and it did not distinguish between individuals with abuse or dependence diagnoses.

Edlund and colleagues (2006) conducted a secondary data analysis from a national stratified probability telephone survey and found that women with SUDs and/or other mental health diagnoses were more likely than men to perceive a need for treatment. The study's sample characteristics were probably closest to the NSDUH sample, thereby potentially providing some indication of what may be found from this study.

Age. It has been suggested that there is a relationship between age and perceived treatment need, but findings are inconclusive. As noted earlier, however, studies show that young adults comprise the age group with the highest levels of alcohol and substance use. Some find younger adults were less likely to perceive a need for treatment (Grella et al., 2009; Wu \& Ringwalt, 2004), while others find they are more likely to perceive a need (Mojtabai et al., 2002) for treatment when compared to older adults. Commentaries on changing trends in substance use suggest that younger and more recent cohorts have 
more accepting views of substance use altogether, as well as social norms which do not label certain degrees of use as problematic; for example, the prevalence of binge drinking among young adults has increased significantly in the past 30 years (Grucza, Norberg \& Beirut, 2009). This may potentially explain why younger cohorts are less likely to perceive a need for treatment, as their use may be comparable of that of their peers.

According to the secondary data analysis of NESARC by Grella and colleagues (2009), men and women comprising the youngest age group (18-29) had the proportionally lowest perceived need for treatment. A secondary data analysis of the 1999 NSDUH focusing on individuals who met criteria for alcohol dependence found that both the youngest adult women and men (18-25) were less likely to perceive a need for treatment than older adult women and men who met criteria for alcohol dependence (Wu \& Ringwalt, 2004). In contrast, findings from the NCS revealed that the youngest group of men and women was most likely to perceive a need for treatment; however this group was comprised of both adolescents and young adults between the ages of 15 and 24 together which may have affected this calculation (Mojtabai et al., 2002).

Race and ethnicity. Research on race and ethnicity in this area is mixed although this may be due to methodological and sample size issues. Additional research with adequate sample sizes can help to clarify whether there is a relationship between race and perceived treatment need, and what that relationship looks like.

Among men and women who met criteria for substance dependence in NESARC, non-white participants were two times as likely to have perceived a need for treatment as white participants (Grella et al., 2009). The comparison groups were relatively large in 
this study, with the responses of 937 white individuals being compared to those of 665 non-white individuals.

No significant racial/ethnic differences were found in the secondary analysis of NCS data of men and women with SUDs (Mojtabai et al., 2002). Yet this study contained a large number of white non-Hispanic individuals $(\mathrm{n}=1380)$ and relatively smaller numbers of members from other groups (176 African Americans, 175 Hispanic individuals and 61 from other ethnic and racial groups) for adequate comparison (Mojtabai et al., 2002).

Conversely, an older study with non-Hispanic white, African American, and Hispanic drug-using men found that minority men were least likely to perceive a need for treatment (Longshore, Hsieh, Anglin \& Annon, 1992). In particular, Hispanic men were less likely to perceive a need for treatment compared to whites, and African Americans were more likely to have negative views about treatment compared to whites. However, the applicability of this study is limited for several reasons. Most importantly, its findings are over 20 years old and cultural attitudes could have changed among younger cohorts. In addition, the sample consisted of men who were recently arrested and who admitted to drug use at that time. The generalizability to a modern day sample of community based substance users is limited, but the findings are still important to consider.

Co-occurring mental health. Studies suggest a strong relationship between cooccurring mental health problems and a perceived need for treatment (Grella et al., 2009; Mojtabai et al., 2002). It appears as though individuals who experience greater impairments in their lives, due to mental health symptomology and substance-related problems, are more likely to believe that they need formal treatment to ameliorate them. 
Conversely, it may be that individuals who experience few co-occurring problems may view their symptoms as more manageable or time-limited, not necessitating formalized treatment.

The perceived need of individuals with co-occurring disorders is also important to understand due to the divided state of treatment in the United States where mental health treatment is separated from substance abuse treatment (Havassy et al., 2009). Individuals with co-occurring disorders who perceive a need for mental health treatment risk having their substance use problems unaddressed or inadequately addressed. There is also some research that suggests gender differences in this area, so that women with co-occurring problems are more likely to perceive a need for mental health treatment than substance use treatment (Green, 2006; Greenfield et al., 2007).

In a review of the NCS data set, it was found that adults with co-occurring mood and substance disorders were two times more likely to perceive a need for treatment than if they had SUDs alone (Mojtabai et al., 2002). Co-occurring anxiety and SUDs led to a similarly high perceived need for treatment as well. Among individuals who met criteria for mood, anxiety and SUDs, the perceived need for treatment was four times as high as for SUDs only. However, the question participants were asked did not clearly ask whether individuals perceived a need for treatment at a substance use treatment facility or with a mental health provider. The question was phrased, "Was there ever a time during the past 12 months when you felt that you might need to see a professional because of your problems with your emotions or nerves or your use of alcohol or drugs?" (Mojtabai et al., 2002, p.78). So despite such significant findings, it is unclear whether its 
implications are for the perceived need for mental health treatment, substance use treatment, or both.

A review of NESARC data revealed that individuals with substance dependence who met criteria for either a mood or anxiety disorder in the past year were more likely to perceive a need for substance use treatment than if they did not (Grella et al., 2009) but this is the only study with community based substance users with such a clear relationship.

Education. This variable was included in the analyses of only one study examining perceived treatment need (Grella et al., 2009). It was found that highest educational attainment was not significantly associated with perceived need for treatment among participants in the NESARC sample who met criteria for substance dependence. This is an area worth further exploring, as those with higher levels of education are generally presumed to be more likely to use health services.

Marital status. Marital status was found to not be significantly associated with perceived need for treatment among participants in the NESARC sample who met criteria for substance dependence (Grella et al., 2009). According to the health care utilization model, marital status usually predicts greater utilization of services (Andersen \& Newman, 1973/2005). This is an area worth exploring in the field of substance use treatment, to see if marriage is associated with perceived need for treatment and, subsequently, greater utilization.

Prior treatment history. Prior treatment history was positively associated with perceiving a need for treatment in a study by Falck and colleagues (2007). However, results were based on their specific sample of rural stimulant drug users. According to the 
theoretical model of the study, prior treatment history and prior illness positively predict future perceived need and utilization of services.

Income. Income was not found to be significantly associated with perceived need for treatment among participants in the NESARC sample who met criteria for substance dependence (Grella et al., 2009). Income was classified as an enabling factor in the theoretical model, suggesting that higher income made access to services easier and more likely to happen. It is yet unclear whether higher income makes substance users more likely to perceive a need for treatment.

Health insurance status. Having health insurance was not significantly associated with the perceived need for treatment among participants in the NESARC sample who met criteria for substance dependence (Grella et al., 2009). However, it is unknown what the association between health insurance status and other levels of substance use problem severity could be. This was viewed as an enabling factor in the theoretical model, yet there is not enough evidence to suggest that having insurance is associated with greater perceived need for treatment among individuals with varying levels of substance use problem severity.

Urban/rural residence. Area of residence was not significantly associated with perceived need for treatment among participants in the NESARC sample who met criteria for substance dependence (Grella et al., 2009). According to the theoretical model, living in an urban setting would make individuals more likely to have greater ease of access to a treatment facility nearby because individuals in rural settings may have fewer facilities nearby. It is worth exploring whether this fact impacts the perception of need for treatment among substance users in different communities. 
In summary, almost all of the factors above which have been explored in the research on perceived treatment need are also present in the theoretical model illustrated in Figure 1, which is why they are also being included for analysis in the current study. As noted, the literature indicates the likelihood that increased problem severity may be related to an increased likelihood to perceive a need for treatment, as well as the presence of co-occurring mental health problems, and for having a prior treatment history. In terms of the remaining variables, they appear to be theoretically meaningful but have not yet accrued enough research evidence to clearly suggest their relationship to increasing or decreasing perceived treatment need. Also, little is known how each of the above-noted variables may be differentially associated with men and women's perceptions of treatment need.

\section{Research Questions and Hypotheses}

The primary aims of this study are to examine (a) the influence of substance use problem severity on perceived treatment need among individuals who meet criteria for SUDs and (b) whether gender has a moderating effect on that relationship. The proposed study will maintain a focus on whether and how gender influences the relationship between substance use problem severity and the perceived need for treatment. However, the association of other predisposing and enabling factors on perceived treatment need will also be explored and gender differences will be examined. The variables for the study were selected from previous research on this topic and the theoretical model presented in Figure 1. Secondary data from the NSDUH on the substance use of 
American household residents will be used in the present study. The following research questions and hypotheses are proposed:

Research Question 1: Is greater substance use problem severity associated with an increased likelihood that individuals who meet criteria for SUDs will perceive a need for treatment compared to lower levels of substance use problem severity?

Hypothesis 1: Individuals who meet criteria for SUDs will be more likely to perceive a need for treatment at greater levels of substance use problem severity compared to lower levels of substance use problem severity.

Research Question 2: Is the gender of individuals who meet criteria for SUDs associated with an increased likelihood of perceiving a need for treatment?

Hypothesis 2: Women who meet criteria for SUDs will be significantly more likely to perceive a need for treatment than men who meet criteria for SUDs.

Research Question 3: Does the gender of individuals who meet criteria for SUDs moderate the likelihood that they will perceive a need for treatment at greater levels of substance use problem severity?

Hypothesis 3: Women who meet criteria for SUDs will be more likely than men to perceive a need for treatment at greater levels of substance use problem severity.

Research Question 4: How do predisposing and enabling individual determinants impact the moderating influence of gender on the likelihood to perceive a need for treatment? Research Question 5: How are predisposing and enabling individual determinants and substance use problem severity associated with the likelihood of perceived treatment need among women? 
Research Question 6: How are predisposing and enabling individual determinants and substance use problem severity associated with the likelihood of perceived treatment need among men?

\section{Significance of Study}

This proposed study makes a contribution to both the substance use treatment and public health literature on the influence of gender upon substance use problem severity and perceived treatment need among community substance users. Unlike other studies on perceived treatment need in the field of substance use, this study proposes a model to be tested in an attempt to better understand the relationship between substance use problem severity, gender, and perceived treatment need. This study is distinguished from other studies in this area also because (a) it includes individuals who use alcohol and/or drugs, (b) it will include substance-using individuals who met diagnostic criteria for three levels of SUD problem severity (abuse, dependence, and polysubstance), (c) the sample size is larger than other studies in this area, (d) it focuses solely upon adults, and (e) it uses a nationally representative sample of individuals. It is also distinguished by its exploration of gender differences in factors which are associated with perceived treatment need.

This is also a significant topic for the field of social work. Social workers and public health workers in various settings such as mental health, corrections, healthcare, and child welfare are likely to encounter individuals with SUDs in their day-to-day work as they help to address other presenting needs (Miller, Forcehimes \& Zweben, 2011). However, the substance use treatment needs of individuals from the community may otherwise be unacknowledged unless proper screening is conducted to identify them 
(Rapp, Dulmus, Wodarski \& Feit, 1999). It is possible that screening may help to identify individuals who perceive a need for treatment, thereby leading the provider to make a referral to treatment.

The use of universal screening for alcohol problems was first recommended by the World Health Organization (WHO) over 30 years ago because it was viewed as a means to identify problem drinkers in an effort to prevent further problems by either encouraging safer levels of drinking or to help them to access specialized substance use treatment (WHO, 2007). Since then, it has also been proven to be effective for individuals regarding their drug use and tobacco use. However, SBIRT has been slow to be implemented among doctors, nurses, and social workers alike due to barriers such as lack of awareness, lack of training, and lack of time (Broyles \& Gordon, 2010; Osborne \& Benner, 2012)

An ample body of research indicates that the evidence-based practice of Screening, Brief Intervention, and Referral to Treatment (SBIRT) (Babor, McRee, Kassebaum, Grimaldi, Ahmed, Bray, 2007) is one of the most effective ways to reach the otherwise overlooked population of community-based substance users (Babor \& HigginsBiddle, 2000; Begun, Rose, Lebel \& Teske-Young, 2009; The Insight Project Research Group, 2009; Madras, Compton, Avula, Stegbauer, Stein \& Clark, 2009; Schonfeld, King-Kallismanis, Duchene, Etheridge, Herrera, Barry \& Lynn, 2010). SBIRT is grounded in the Transtheoretical Model and entails the use of standardized screening measures to assess drug and alcohol use, followed by the delivery of a brief motivational intervention (2-6 sessions) if a possible problem is detected (Babor et al., 2007). Sometimes this is enough and research shows that even a brief conversation has led to 
decreases in problematic drinking behaviors at follow-up (Babor \& Higgins, 2000). However, if the client is interested, a referral to treatment can then be made. Research on SBIRT has been conducted with various populations around the world and it is increasingly being used by doctors, nurses, and social workers in diverse settings (Begun et al., 2009; InSight Project Research Group, 2009). Studies suggest that SBIRT is a promising and cost-effective method to identify problematic substance users and to help improve health (Madras et al., 2009). It is also quite timely to be discussing the use of SBIRT, as a recent press release from SAMHSA (2012b) announced that $\$ 22$ million has been allocated to increase the use of this approach in primary care facilities and other community health settings.

This research has the potential to facilitate gender-informed outreach and public health messages to adults who meet criteria for SUDs who may be experiencing problems due to their use. By better understanding the factors which are associated with a perceived need for treatment, treatment agencies can be better equipped to promote their programs to people who may be receptive to receiving formalized substance use treatment. The results of this study could assist social workers in developing gendersensitive policies and programs focused on reducing problematic substance use among adults in the community. 


\section{CHAPTER III. METHOD}

\section{Research Design}

The study used a cross-sectional research design and was conducted using secondary data drawn from a national survey. Data from the 2010 annual survey was specifically chosen because it was the largest and most up-to-date data set available for public use which included participants from all 50 states. The study analyzed data collected through audio computer-assisted personal or self-interviews conducted at each participant's place of residence. The following variables were examined: (a) perceived treatment need 12 months prior to the assessment, (b) substance use problem severity in the 12 months prior to the assessment, (c) participant gender, (d) co-occurring depression in the 12 months prior to the assessment, (e) co-occurring anxiety in the 12 months prior

to the assessment, (f) participant race and ethnicity, (g) participant age, (h) education, (i) any prior lifetime treatment history, (j) total family income 12 months prior to the assessment, (k) current health insurance status, and (k) current urban/rural residence.

\section{NSDUH Design}

The data source for the proposed research is the public use file of the 2010 National Survey on Drug Use and Health (NSDUH) (United States Department of Health and Human Services, SAMHSA, Center for Behavioral Statistics and Quality, 2011). It is an annual cross-sectional survey which has been conducted for 30 years. It aims to provide estimates of the lifetime and recent substance use of Americans over the age of 12 , as well as correlates such as treatment utilization and other aspects of physical and 
mental health. Demographic information is also gathered, including marital status, educational level, household composition and other characteristics. It primarily targets a non-clinical sample of household residents and those living in other group quarters, including civilians on military bases. Equal numbers of participants are gathered from the following age groups, so that each comprises one third of the sample: 12-17 years, 18-25 years and 26 years or older. (There is a deliberate attempt made to oversample the number of participants aged 25 and younger.) The 26 years or older group is then divided into thirds and comprised of equal numbers of participants between 26 and 34 years, 3549 years and 50 years or older. Data were collected and prepared by the Research Triangle Institute (RTI).

Sampling Strategy. The NSDUH consists of an independent, multi-stage area probability sample to capture responses from participants in all 50 states and the District of Columbia. Eight states (California, Florida, Illinois, Michigan, New York, Ohio, Pennsylvania and Texas) were determined to be large sample states where each contributed 3,600 participants to the survey sample. The remaining 43 states contributed 900 participants each to the survey sample. Then each of the 51 states was broken up into almost geographically equal-sized regions called state sampling regions. The large sample states were each divided into 48 state sampling regions while the other 43 states were divided into 12 state sampling regions each. This resulted in a total of 900 state sampling regions across the United States. Sampling units were divided according to: core-based statistical areas (metropolitan and surrounding areas with populations over $10,000)$, socioeconomic status, and the percentage of the population who were nonHispanic white. Then 48 census tracts were found in each state sampling region. Next, 
census tracts were divided into smaller geographic segments by merging neighboring census blocks. Dwelling units were selected within each segment and then participants were selected per dwelling based on age criteria.

Data were collected through interviews conducted at the participant's place of residence. The interviews consisted of both computer-assisted personal interviewing by an interviewer and also audio computer-assisted self-interviewing for more sensitive questions. Interviews were conducted in either English or Spanish, according to the participants' preference. Participants were given $\$ 30$ for their participation in the survey.

The final sample size of the entire 2010 NSDUH included 68,487 participants, based on a weighted interview response rate of $74.7 \%$. The weighted interview response rate is calculated by dividing the number of interviews actually completed by the number of individuals who were selected to interview. The public use file contains 57,873 participants due to additional steps which were meant to protect anonymity as well as removal of cases deemed non-usable due to significant numbers of missing responses.

The NSDUH incorporates the use of DSM-IV diagnostic criteria to determine whether a participant has met criteria for a SUD, this was done through using the Structured Clinical Interview for DSM-IV-TR Axis I Disorder Non-Patient Edition (SCID). In addition, other standardized measures were incorporated into the survey to assess mental health symptom severity and other characteristics such as the World Health Organization Disability Assessment Schedule (WHODAS), the K6 mental health screening instrument, and the Sheehan Disability Scale (SDS).

Missing Data. Missing data are important to identify and address in any survey because it impacts the ability of the researcher to make sound inferences based on their 
findings (Schafer \& Graham, 2002). There are two types of missing data which impact statistical analyses on survey data: unit nonresponse and item nonresponse. In cases of unit nonresponse for a survey, an individual eligible for the study may choose not to participate altogether or be unavailable for participation. This issue can negatively impact surveys because they can reduce the representativeness of the sample to the general population, especially if people with certain shared characteristics are underrepresented in the sample. In situations with item nonresponse, a participant may not provide all necessary responses for all questions so there are some items with missing values. Item nonresponse can impact outcomes because it means that there is less original data provided from the participant to draw conclusions from. Fortunately, there are statistical analyses and techniques to adjust for these types of missing data and the public use data set came with many of these adjustments already made.

Missing data due to item nonresponse was addressed through person-level weighting, which will be briefly discussed in the section on weighting. Occasional missing data due to item nonresponse on core questions was addressed by RTI in either one of two ways; either through logical assignment or statistical imputation. Logical assignment was done when the participant had provided enough data in other questions so that the question missing data could be logically answered by drawing from prior responses. In other circumstances, missing data were addressed through statistical imputation. Predictive mean neighborhood (PMN) was the imputation procedure used. This procedure had been specifically developed and used for prior versions of the NSDUH. It involved both using predictive values from a model, as well as randomly from another participant in the sample with similar characteristics. These assignments 
were conducted by RTI and new variables (usually with the same name but with the prefix "IR") were created within the public use data set to distinguish those which had been imputed from those left with missing values. This study utilized IR items when available for variables.

"Usable data." The survey analysis team at RTI also made determinations on "usable" versus "nonusable" cases and only usable cases were subjected to imputation procedures when there were issues with item nonresponse. It is only these usable cases which were also meant for analyses in the public use data set (RTI, 2012). Criteria for determining whether cases were usable were based upon the amount of missing data due to item nonresponse, because high levels of missing data would require extensive amounts of imputation methods to be implemented to move forward. In addition, nonusable cases were those which responded negatively to "gate" questions on lifetime use or non-use of any mood-altering substances, including licit substances such as tobacco products or alcohol, or illicit/non-prescribed substances. In particular, participants had to provide information about lifetime use or nonuse of cigarettes and at least nine of the following substances: chewing tobacco, snuff, cigars, alcohol, marijuana, cocaine, heroin, hallucinogens, inhalants, pain relievers, tranquilizers, stimulants, and/or sedatives.

Weighting procedures. The findings from the NSDUH aim to be representative of the entire population of Americans over the age of 12, however it only surveys a much smaller sample of this population. In order to achieve unbiased estimates of the population based on the sample participants, person-level weights were calculated by RTI. The final sample weight variable was provided in the public use data set 
(ANALWT_C). This was applied to cases in the data set by using the weight function in SPSS and specifying that the weighting variable should be applied to all cases. This would then serve to make each participant represent other members of the population who share characteristics with them. The weight applied to each case is unique and is the product of 15 weight components; which account for various factors such as sampling strategy, nonresponse, extreme responses, and census track data from 2000. As a result of applying the weighting variable, the unweighted sample of 5,484 participants became representative of 20,077,235 Americans in the general population. Annual reports by SAMHSA which summarize NSDUH results provide findings from the weighted sample only, in order to provide estimates which are representative of the American noninstitutionalized population over the age of 12 (SAMHSA, 2010). More information on weighting procedures can be found in the 2010 NSDUH Methodological Resource Book (Chen et al., 2012).

Strengths and limitations of NSDUH. The NSDUH is characterized by several important strengths, particularly its large sample of American residents living in all 50 states. The use of audio computer-assisted technology helped to maintain participants' privacy and to reduce social desirability in responses that sometimes occur during faceto-face interviews which asked questions about sensitive issues such as substance use and mental health symptoms (De Leeuw, Hox \& Kef, 2003). The use of computer-assisted technology also ensured that all interviews were conducted in a standardized manner for the entire survey. In addition, to account for the growing numbers of individuals who have used prescription medications, pill flashcards were used to assist participants with recall and identification of prescription medications that they may have used. 
There are also some limitations to using the NSDUH survey data. The sample of the NSDUH is not a purely random sample of American household residents. Deliberate efforts were made to ensure residents in less populous states were still represented. In addition, participants under the age of 26 were overrepresented in the sample. The survey was purely quantitative and relied solely upon self-reports for data. This means that a great deal of rich, subjective information could not be gathered, as would be possible in a qualitative or mixed methods design. Self-reports can be influenced by issues with recall and social desirability, thereby limiting their validity and reliability. There was no record which indicated that the NSDUH required participants to complete a social desirability scale in order to address this response bias. Also, the NSDUH is a cross-sectional survey which gathers information about a single cohort of subjects at only one point in time. Cross-sectional surveys are limited in the causal inferences that can be made based upon their data. The exclusion of institutionalized populations and homeless individuals can affect the data gathered because prison and substance use treatment populations may have higher substance use prevalence rates which may impact total estimates. In addition, the survey did not ask about documentation or citizenship status, so it is unclear whether undocumented immigrants or other residents were adequately represented in the sample.

\section{Data Management}

The NSDUH 2010 public use data set for the study was made available for download free of charge from the Substance Abuse and Mental Health Data Archive online (http://www.icpsr.umich.edu/icpsrweb/SAMHDA/studies/32722). No application or registration was needed, other than the agreement that confidentiality of research 
participants would be maintained. It was understood that if confidentiality of participants was breached, that various sanctions could be implemented.

The data were downloadable from the website in SPSS format, with separate files for codebooks, questionnaires and other related literature. Within the SPSS data file, responses for 57,873 participants were included along 3,112 variables (including recoded and imputed values). These variables fell into three basic categories: core substance use variables (such as frequency and method), non-core substance use variables (including consequences due to use, health issues, mental health, among others), and demographic variables. Each participant on the public use file had already been assigned a unique ID number for confidentiality.

\section{Study Sample}

The study's sample was selected from the NSDUH sample. Adult participants who met criteria for at least one SUD in the past year ( $n=20,077,235$ weighted sample; $n=5,484$ unweighted sample) comprised the study sample. One group consisted of participants who perceived a need for substance use treatment in the past year and the other group consisted of participants who did not perceive a need for substance use treatment. Demographically, the sample consists of men and women over the age of 18 who reside in all 50 states and the District of Columbia. Although there are several studies in the literature that measure the perceived treatment need of substance users (Grella et al., 2009; Grella et al., 2011; Mojtabai et al., 2002; Wu \& Ringwalt, 2004), none have explored the potential differences between those who meet criteria for abuse, those who meet criteria for dependence, and those who meet criteria for multiple SUDs. 
The examination of how different levels of substance use problem severity may influence perceived treatment need is an important element of the research design as it has not been studied in this manner and may reveal why such small proportions of community samples of substance users generally do not perceive a need for treatment, especially if they do not experience substance use problems with great severity. Therefore, the inclusion of substance users who experience varying levels of substance use problem severity can allow for a better understanding of its effect on perceived treatment need.

Perceived treatment need. Participants were asked, "Do you think you needed treatment for in the past year?" (The computer program automatically filled in the name of the substance (s) that the participant reported using within the past 12 months for the question.) Responses could be either "yes" or "no." Participants who responded "no" because they did not believe that they needed treatment within the past 12 months were coded as 0 , for not perceiving a need for treatment. This group consisted of 19,462,974 participants $(5,243$ unweighted participants). Those who responded "yes" that they believed they needed treatment in the past 12 months were coded as 1 , for perceiving a need for treatment. This group consisted of 986,498 participants (241 unweighted participants).

Substance use problem severity. The criteria for determining substance use problem severity came from the fourth edition text revision of the widely used Diagnostic and Statistical Manual for Mental Disorders (DSM-IV-TR) (American Psychiatric Association, 2000). Participants were asked questions to determine whether they met any of the four criteria for substance abuse or the seven criteria for substance dependence for each substance they reported using on more than five days within the past year. For 
example, one of the questions which would be asked to a participant who reported alcohol use within the past year was, "During the past 12 months, was there a month or more when you spent a lot of your time getting or drinking alcohol?" If the participant responded 'yes' to this question and two of the other seven criteria for alcohol dependence, the participant then was coded as meeting criteria for alcohol dependence.

Substance use problem severity was a recoded variable created by the author in which the three mutually-exclusive levels had to be distinguished from one another because the data set did not make these distinctions. The data set identified whether participants met criteria for each of the possible SUDs (e.g. cocaine abuse, opioid dependence) but did not distinguish between them any further. The creation of the substance use problem severity variable was a multi-step process due to the manner in which SUDs were coded in the pre-existing data set. First, the author identified all cases in which criteria were met for a single abuse diagnosis only. These cases were labeled as 'low' problem severity in the newly created 'substance use problem severity' variable. Next, the author identified all cases where criteria were met for a single substance dependence diagnosis only and these cases were labeled 'moderate' problem severity in the 'substance use problem severity' variable. Lastly, the author labeled all remaining cases as 'high' problem severity in the 'substance use problem severity' variable because they were cases in which criteria were met for abuse or dependence for multiple substances. The low severity cases were coded as 0 , the moderate severity cases were coded as 1 , and the highest severity cases were coded as 2 . This approach to determining substance use problem severity, particularly for alcohol, has been documented as a more efficient means for determining problem severity rather than measuring it based on 
numerical counts of symptoms experienced (Dawson \& Grant, 2010; Dawson, Saha \& Grant, 2010)

The DSM-IV currently details diagnostic criteria related to the following substances: alcohol, amphetamines, caffeine, cannabis, cocaine, hallucinogens, inhalants, nicotine, opioids, phencyclidines, sedatives, hypnotics and anxiolytics. There are two levels of diagnosable SUDs: abuse and dependence. A diagnosis of either substance abuse or dependence is, first and foremost, marked by "a maladaptive pattern of substance use leading to clinically significant impairment or distress" (APA, 2000, p. 175-272).

A substance abuse diagnosis requires a minimum of one of the four following criteria within a single 12-month period: ongoing substance use which impacts one's ability to fulfill obligations at work, school or home; ongoing substance use in physically hazardous situations; legal problems as a result of substance use; or ongoing substance use even though it has caused problems in relationships or has negatively impacted relationships. This diagnosis does not generally require intensive treatment and can often be addressed on an outpatient basis in a time-limited manner. It is viewed as the less severe of the SUDs.

A substance dependence disorder is diagnosed when an individual meets three or more of the following criteria within a 12-month period: physiological tolerance; physiological withdrawal; frequently consuming more of a substance in an occasion than was initially intended; unsuccessful attempts to reduce or stop using the substance; acquiring the substance, consuming the substance and recovering from the substance takes up a great deal of the person's time; the person no longer participates in social, 
occupational or enjoyable activities because of their substance use; or the person continues to use the substance although they may have a physical or psychological problem that is either caused by or negatively impacted by their substance use. Substance dependence is a more severe problem than abuse, frequently warranting more intensive and longer-term treatments. In common parlance, a substance dependence diagnosis is often equated with "addiction" although that word appears nowhere within the DSM-IVTR. There are two basic typologies of dependence: dependence with physiological dependence or dependence without physiological dependence. Treatments for physiological dependence to substances (specifically, opioids, alcohol and benzodiazepines) sometimes utilize medication therapies to manage withdrawal symptoms and ongoing cravings.

Gender. Participants answered questions about their gender as part of the survey, identifying as either 'male' or 'female' so that these dichotomous responses were included in the analyses.

Covariates. In addition to the aforementioned key variables, covariates that may influence of the predictors and outcome variable were explored. These covariates were chosen in accordance with the theoretical model. The predisposing individual determinants of health service utilization which were included as covariates are age, marital status, past substance use treatment, education, and race/ethnicity. The enabling factors which were included as covariates include income, health insurance status, and county type. In addition, research suggests that co-occurring mental health problems do increase the likelihood of perceived treatment need, so the variables on past year anxiety and past year depression were also included. There is also not enough evidence to clearly 
illustrate whether predisposing individual factors such as education or prior treatment history are associated with perceived treatment need or whether enabling factors such as income, health insurance status, and urban/rural residence impact the perceived need for treatment among substance users. Exploring these variables with the large, diverse sample in this study could provide valuable information on the association of these variables with perceived treatment need.

Age. The NSDUH data set came with participants grouped according to age group, $18-21,22-25,26-29,30-34,35-49,50$ to 64 , and 65 or older. Specific ages were not provided for each participant in the public use file after the de-identification process.

Race and ethnicity. Participants were asked their race and whether they identified as ethnically Hispanic in this survey. These two pieces of information were combined for the race/ethnicity variable. For analyses, participants were maintained in the seven categories used by the NSDUH: non-Hispanic white, non-Hispanic black, Native American/Alaska Native, Native Hawaiian Islander/Other Pacific Islander, Asian, nonHispanic bi- or multiracial, and Hispanic.

Past year anxiety. Anxiety was included in a list of other mental health and physical health conditions that the participants were asked about. They were presented with the following statement, "The following is a list of health conditions. Please read the list, and type in the numbers of any of these conditions that a doctor or other medical professional has told you that you had in the past year." Participants who selected anxiety were recorded as having experienced anxiety in the past year. 
Past year depression. The variable for past year depression underwent a similar process as anxiety and other health conditions. Participants who selected depression from the list of diagnosed health concerns from the past year were recorded.

Education. Information about each participant's highest completed degree was included in the NSDUH, however this study will simply distinguish between those who did or did not complete high school. This will be a dichotomous variable to classify individuals as without a high school diploma or those with a high school diploma, as was done by Grella and colleagues (2009).

Prior treatment history. Participants were asked, "Have you ever received treatment or counseling for your use of alcohol or any drug, not counting cigarettes?" This was recorded as a dichotomous yes/no response.

Total Family Income. This was a categorical variable, with income brackets already created in the public use data file for those who responded incomes between $\$ 0$ $\$ 19,999, \$ 20,000-\$ 49,999, \$ 50,000-\$ 74,999$, and those with incomes above $\$ 75,000$.

Health insurance status. This is a dichotomous variable in the survey, summarizing whether participants either reported having any type of health insurance (private or public).

County of residence. This was a variable in the survey in which participants were classified as residing in large metropolitan areas, small metropolitan areas, or nonmetropolitan areas based upon the sampling strategy of NSDUH.

Marital status. This was a categorical variable in the survey with three possible responses: (1) never married, (2) widowed, divorced, or separated, and (3) married. 


\section{Protection of Human Participants}

The following pieces of information were immediately removed by the Research Triangle Institute to preserve confidentiality of participants before releasing the data set for public use: all directly identifiable information, census region, state, other geographic identifiers, and links between participants in the same household. In addition, RTI's team ensured the full file underwent RTI's own patented procedure to further eliminate identifiable information. Their procedure is known as MASSC (Micro Agglomeration, optimal probabilistic Substitution, optimal probabilistic Subsampling, and optimal sampling weight Calibration) (for more information on this procedure, refer to RTI's webpage: $\underline{\text { http://www.rti.org/page.cfm?nav=736) }}$

Because this dataset underwent such extensive steps to reduce or eliminate personally identifying information before being made available for use to the public, this proposed study does not present a risk to any human participants. This was confirmed by the Florida International University Institutional Review Board, which determined that the study was exempt from review.

\section{Data Analysis Plan}

Descriptive statistics. Frequencies were tabulated for the entire sample, both weighted and unweighted, to see the characteristics of the sample along all demographic variables. Frequencies were also tabulated and stratified by perceived treatment need and gender to view the differences in proportion along demographic characteristics. Within both the perceived treatment need group and the no perceived treatment need group, omnibus chi-square tests for homogeneity were conducted along gender to note any 
statistically significant differences between gender at each variable. The omnibus chisquare tests do not identify which levels are different, however. Cramér's $V$ was calculated to determine the effect size of the relationships between variables. Small effect sizes were considered have values over the cut-off of 0.10 , moderate effects were over 0.30, and large effects were over 0.50 (Abbott \& McKinney, 2013). Post hoc chi-square tests were then conducted to further evaluate group differences by gender on variables with Cramér's $V$ effect sizes greater than 0.10 .

Reasons for not seeking treatment. In addition, an exploratory analysis was conducted with a variable found in the data set regarding the reasons why participants who perceived a need for treatment in the past year did not seek treatment. A frequency table was constructed in which participant responses to this question were summarized. Separate tables were also made and separated by gender to determine which responses were most common for each gender.

Participants who reported perceiving a need for treatment were asked the following question two questions: "During the past 12 months, did you make an effort to get treatment or counseling for your use of __ ? "Which of these statements explain why you did not get the treatment or counseling you needed for your use of ?" (The computer program automatically filled in the name of the substance (s) that the participant reported perceiving a need for treatment for.) Participants could check more than one statement for this question.

Checking for assumptions of logistic regression. Statistical analyses were completed using SPSS 19.0 and Stata 12 statistical software to test for assumptions of logistic regression and to conduct the analysis itself. For one, the sample size should be 
adequately large with enough cases in each category to allow for necessary calculations and comparisons between groups, especially in studies with numerous predictor variables (Pallant, 2007). This was not a problem for the current data set given that there were over 50 cases per predictor in the model, even before weighting (Burns \& Burns, 2009).

In addition, there should be no multicollinearity between predictor variables in the model because logistic regression analyses are sensitive to inter-predictor correlations and it can affect results (Salkind, 2003; Pallant, 2007). Multicollinearity leads to inaccurate estimates of coefficients and standard errors as well as inference errors concerning findings (Tabachnick \& Fidell, 2007). In order to test for multicollinearity, various correlation coefficients were calculated between the variables, as some were nominal and others were ordinal. Four different correlation tests (Spearman's rho, phi coefficients, Cramer's V, and rank-biserial) were conducted, depending on the variables being analyzed. Spearman's rho, phi coefficients, and Cramer's V were conducted using SPSS. Rank-biserial was conducted using Stata. The correlation cut-off to screen for multicollinearity was $r=0.90$ between variables (Tabachnick \& Fidell, 2007).

Spearman's rho correlation coefficients were calculated between only ordinal variables (substance use problem severity, age, county of residence, and income) (Brace, Kemp, \& Snelgar, 2009). Phi coefficients were calculated to test for correlations between only dichotomous nominal variables (gender, past year anxiety, past year depression, prior substance use treatment, insurance status, education, and perceived treatment need) (Jackson, 2012). Phi coefficients range between -1 and +1 so that strong relationships between variables are closer to 1, as in the case of Spearman's rho. Cramer's V coefficients were calculated when nominal variables with three or more levels 
(race/ethnicity and marital status) were tested with the previously noted dichotomous nominal variables. Cramer's V coefficients were also calculated between ordinal variables and the nominal variables with three or more levels. Strong associations between variables are reflected by Cramer's V coefficients closer to 1. Rank biserial correlation coefficients (Somer's d) were calculated when ordinal variables were correlated with dichotomous nominal variables. If multicollinearity was found between predictor variables, a composite variable would have been created.

Logistic regression analyses are sensitive to outliers, which can negatively impact goodness of fit of the model and incorrectly classify cases along the dependent variable (Pallant, 2007). They can be identified in two ways. They can be found within the classification plot which highlights false positives and false negatives (Burns \& Burns, 2009). They can also be identified through examining standardized residuals in the case wise list for values that are greater than 2.5 or less than -2.5 (Pallant, 2007). All of the perceived treatment need cases could have been classified as outliers because they were found within the classification plot and had high standardized residual scores, likely due to their relative infrequency in the sample. However, they were not excluded from the analyses because they were necessary for comparison.

Logistic regression. A binary logistic regression using the forced entry method was conducted using SPSS software (version 19) to test each hypothesis in the study. This method was used rather than sequential logistic regression because it was not hypothesized that the order of the predictor variables differentially impacted the dependent variable (Tabachnik \& Fidell, 2007). Stepwise logistic regression was not appropriate for this study because all of the predictor variables were theorized to impact 
the dependent variable and stepwise logistic regression is better suited for exploratory studies for theory development to identify which predictors have the greatest impact on the model.

Logistic regression was selected for the primary analysis because of the presence of a categorical dependent variable, perceived treatment need. In addition, logistic regression provides the opportunity to both predict participant group membership and to understand the degree of relationships between the predominantly categorical variables, which would not have been possible with discriminant analysis (Brace et al., 2009; Burns \& Burns, 2009). By conducting a logistic regression, one is be able to estimate the log odds that a participant will perceive a need for treatment.

When interpreting outputs from logistic regression analyses, one can interpret $B$ coefficients (the slope values), odds ratios, and/or probabilities in percentages. For the purpose of this study, the researcher will discuss odds ratios and their confidence intervals only for ease of interpretation although $B$ coefficients will be presented in tables summarizing model outputs directly from SPSS. It should be noted that log odds ratios are different than standard odds ratios because they can range from negative infinity to positive infinity. When a log odds ratio equals the number one for a main effect analysis (the first two research questions of this study are for main effects), it means that there is not a statistically significant difference between cases on the dependent variable. The third and fourth research questions of this study are for the interaction effect of gender on the relationship between substance use problem severity and perceived treatment need. Different values for the odds ratios at moderate and high substance use problem severity will indicate that the effect of substance use problem severity varies based on the 
participant's gender. If the odds ratios at both levels of substance use problem severity are the same, then it can be said that there is no interaction effect. (Jaccard, 2001).

Because this was a secondary data analysis, the researcher was already aware that there was a large discrepancy in perceived need for treatment in the sample, with only $4.8 \%$ of the sample perceiving a need for treatment and the remaining $95.2 \%$ who did not. This is why confirmatory analyses were also conducted using Stata statistical software and Relogit software for rare events logistic regression (King \& Zeng, 2001a; 2001b). Relogit software has been specifically developed for rare events data in which the smallest subgroup for analyses is as small as $1 \%$ of the size of the largest subgroup for analyses. Using this software was meant to help to prevent estimated event probabilities from being too small and from errors also being biased in that direction. As noted earlier, logistic regression is sensitive to outliers and this can lead to both inaccurate coefficient estimates and odds ratios. The software allows for correction to the bias, thereby reducing error variance. Relogit software calculates more robust standard errors than traditional logistic regression, thereby also reducing $\mathrm{z}$ scores, and leading to potentially larger $p$ values. Coefficients calculated through the standard logistic regressions were compared to those coefficients calculated using Relogit software in order to check for potential underestimation bias. In cases where a discrepancy was found between coefficients, Relogit coefficients would be used.

The logistical regression analyses were conducted on the weighted sample using the "enter" method in order to examine the hypothesized predictors of perceived treatment need (Pallant, 2007). The first two research questions were addressed by conducting simple binary logistic regression analyses to measure main effects of single 
predictor variables. The third research question was examined by entering both predictors (substance use problem severity and gender), and their corresponding interaction terms in a single block. To explore the fourth research question, all predisposing and enabling factors (e.g. age, co-occurring mental health, race/ethnicity), substance use problem severity, gender, and both interaction terms were entered into the equation in a single block. The interaction terms for the last two research questions were created by multiplying the predictor variable of substance use problem severity and the moderator variable; gender (Frazier, Tix \& Barron, 2004). This thereby resulted in two interaction terms, gender $\mathrm{x}$ moderate problem severity and gender $\mathrm{x}$ high problem severity. The creation of interaction terms in this manner is the standard when testing moderation with dummy coded variables in a logistic regression model (Jaccard, 2001). The moderation hypothesis was tested when both interaction variables were entered into the equation to determine whether gender moderates the relationship between problem severity and perceived treatment need. This same process was replicated when using Relogit in Stata.

Post-hoc power analysis. A power analysis was conducted in order to determine the likelihood that the null hypothesis would be correctly rejected (Faul, Erdfelder, Lang \& Buchner, 2007). This is important because there is always the chance of error when using statistical tests- either rejecting the null hypothesis when it is true (known as a Type I error) or failing to reject the null hypothesis when it is false (known as a Type II error). The software PASS 11, a statistical power analysis software, was used to estimate power for the proposed study (Hintze, 2011).

Post-hoc power analyses were conducted for the proposed study because the study's sample size was already known. In the behavioral and social sciences, it is 
common to strive for a power of $80 \%$ so that was the percentage used for the proposed study. The significance level was set at $0.10 \%$ probability of result not being true $(\alpha=$ 0.001). The sample size was $20,077,235$ participants. The odds ratio was set to 1.50 . This estimate represents a large effect size estimate (i.e., an odds ratio of 1.50 will indicate that each standard deviation increase in a predictor will be associated with a $50 \%$ increase in the likelihood to perceive a need for treatment). Upon calculation, it was found that the power of the sample was 1.00 and adequate for analyses due to an exceptionally large sample size even prior to weighting. 


\section{CHAPTER IV. RESULTS}

This study, utilizing logistic regression analyses, aimed to understand the relationship between substance use problem severity and perceived treatment need among community-based substance users and to determine whether gender moderated that relationship. As described in the previous chapter, the data analytic plan required several preliminary analyses to be conducted in order to test for the assumptions of a logistic regression, as well as descriptive analyses on demographic characteristics and other predictor variables, missing data analyses, and checking for bivariate correlations between predictor variables. Next, the logistic regression was conducted in four blocks so as to include the covariates, substance use problem severity, gender and the interaction term to test for the moderation hypothesis. The logistic regression was then confirmed using Stata software with the Relogit plug-in due to the disparate number of participants who perceived a need for treatment.

\section{Descriptive Statistics}

\section{Overall sample characteristics.}

All descriptive statistics were conducted using SPSS. Table 1 summarizes the descriptive statistics (both weighted and unweighted) for all variables included in the models. Due to the fact that all included variables were categorical, characteristics are summarized in terms of frequencies in percentages. Frequencies are presented for the unweighted sample $(n=5,484)$ and for the sample after weighting was applied $(N=20,077,235)$. There were generally few major differences between unweighted and 
weighted sample proportions along variables, with some notable exceptions (nonHispanic black ethnicity and race, all three levels of marital status).

\section{Stratified sample characteristics.}

Table 2 provides descriptive statistics for the weighted sample which are stratified by perceived treatment need as well as for gender because of the study's emphasis on these particular variables. Tables 3 and 4 provide frequencies cross-tabulated by gender for the perceived treatment need and no perceived treatment need groups respectively, and omnibus chi-square tests for homogeneity. The chi-square test statistics, Cramér's $V$ (effect size), and frequencies will be detailed by each variable and compared within groups.

Gender. A chi-square test was conducted to determine whether gender proportions were equal across both the perceived need for treatment group and the group which did not perceive a need for treatment, $\chi^{2}(1, N=20,449,472)=39,545.80, p<0.001$, Cramér's $V=0.044$. The test revealed that proportions were significantly different, however with a negligible effect size just barely over 0 . A review of Table 3 shows that there were almost equal numbers of men and women who perceived a need for treatment and Table 4 shows that $60 \%$ of those who did not perceive a need for treatment were men.

Substance use problem severity. Table 3 presents the chi-square test of homogeneity which revealed that proportions of substance use problem severity were not equal across genders in the perceived need for treatment group, $\chi^{2}(2, N=986,498)=$ $12,212.77, p<0.001$, Cramér's $V=0.111$. The test revealed that proportions for each level 
of problem severity were significantly different by gender, with the effect size coefficient revealing a small association between variables. Due to the small effect size, post-hoc chi-square tests were conducted to determine which levels differed significantly from each other by gender. The post-hoc test revealed that men and women in the sample differed significantly between the moderate and high levels of substance use problem severity $\chi^{2}(1, N=986,498)=12,179.17, p<0.001$, Cramér's $V=0.120$.

Table 4 presents the omnibus chi-square test values for the group of participants who did not perceive a need for treatment by gender. The group also had unequal representations of each gender at different levels of substance use problem severity, $\chi^{2}(2$, $N=19,462,975)=50,389.01, p<0.001$, Cramér's $V=0.051$ with virtually no effect between the variables.

Table 2 shows that frequencies of substance use problem severity appeared to differ more notably between the perceived need for treatment group and those who did not perceive a need for treatment rather than within each group by gender. In general, it appeared as though there were far more participants with moderate (47.5\%) and high (38.9\%) problem severity who perceived a need for treatment while more participants with low (45.8) and moderate (38.7) severity problems comprised the group which did not perceive a need for treatment. There was a great degree of problem severity overlap between each as well, with almost $40 \%$ of each group meeting criteria for moderate substance use problem severity.

Race and ethnicity. Statistically significant differences in proportions were found for race and ethnicity and gender for both groups, as revealed in Tables 3 and 4 . However 
only the perceived treatment need group (Table 3 ) revealed an almost medium-sized effect between race and ethnicity and gender.

A series of post-hoc chi-square tests were conducted by gender and racial/ethnic groups in order to determine where the largest differences were. The test conducted to see the gender differences between non-Hispanic whites and non-Hispanic blacks revealed a small effect $\chi^{2}(1, N=986,498)=11,984.34, p<0.001$, Cramér's $V=0.130$. A small effect was also found when gender differences between participants identified as non-Hispanic whites and Native Hawaiian/Pacific Islanders were compared $\chi^{2}(1, N=986,498)=$ 25,048.23, $p<0.001$, Cramér's $V=0.199$. A small effect was found when gender was contrasted between non-Hispanic black and Native American/ Alaskan Native participants, $\chi^{2}(1, \mathrm{~N}=986,498)=9,818.83, \mathrm{p}<0.001$, Cramér's V=0.246. A large effect was found when non-Hispanic black participants and Native Hawaiian/ Pacific Islanders were compared by gender $\chi^{2}(1, N=986,498)=39,409.82, p<0.001$, Cramér's $V=0.563$. A medium size effect was found when non-Hispanic black participants were contrasted with biracial and multiracial non-Hispanic participants $\chi^{2}(1, N=986,498)=17,937.08, p<$ 0.001 , Cramér's $V=0.348$. Perceived treatment need differed significantly between nonHispanic black participants and Hispanic participants by gender $\chi^{2}(1, N=986,498)=$ $14,951.76, p<0.001$, Cramér's $V=0.248$. Gender was associated with significant difference between participants who identified as Native American/ Alaska Native compared to those who were Native Hawaiian/Pacific Islander $\chi^{2}(1, N=986,498)=$ 16,574.73, $p<0.001$, Cramér's $V=0.436$. An association was found when gender differences were analyzed between Native American/Alaska Native participants and Asian participants $\chi^{2}(1, N=986,498)=2,127.33, p<0.001$, Cramér's $V=0.182$. A small 
gender effect was found when Native American/ Alaska Native participants were compared to biracial and multiracial non-Hispanic participants. An extremely large gender effect was found when Native Hawaiian/ Pacific Islander participants were compared to Asian participants $\chi^{2}(1, N=986,498)=26,400.00, p<0.001$, Cramér's $V=1.00$. Racial/ethnic and gender associations were found between Native Hawaiian/ Pacific Islander participants and biracial and multiracial non-Hispanic participants $\chi^{2}(1$, $N=986,498)=10,576.31, p<0.001$, Cramér's $V=0.381$. Significant associations were found by gender when comparing Native Hawaiian/Pacific Islander participants to Hispanic participants $\chi^{2}(1, N=986,498)=18,021.61, p<0.001$, Cramér's $V=0.327$. Small but significant associations were also found when Asian participants were compared to biracial and multiracial non-Hispanic participants $\chi^{2}(1, \mathrm{~N}=986,498)=3,392.30, \mathrm{p}<$ 0.001, Cramér's V $=0.260$. Asian participants and Hispanic participants also differed significantly by gender among those who perceived a need for treatment $\chi^{2}(1$, $N=986,498)=2,197.95, p<0.001$, Cramér's $V=0.123$.

Upon reviewing Table 3 it appears as though there were no men who selfidentified as Native Hawaiian or Pacific Islander who perceived a need for substance use treatment and no Asian women who perceived a need for treatment. In addition, twice as many non-Hispanic black men perceived a need for treatment compared to similarly identified women and twice as many non-Hispanic multiracial women perceived a need for treatment compared to men who identified with a similar background.

Age. Statistically significant differences in proportions were found for gender and age in the perceived treatment need group, along with an almost medium-sized effect as shown in Table 3. Post-hoc chi-square tests revealed some differences between certain 
age groups by gender. Gender differences were found between the youngest age group $(18-21)$ and those participants between ages 30 and $34 \chi^{2}(1, N=986,498)=33,483.48, p$ $<0.001$, Cramér's V $=0.303$. There were significant gender differences also found between the youngest age group at those between 50 and $64 \chi^{2}(1, N=986,498)=$ 21,472.43, $p<0.001$, Cramér's $V=0.270$. A medium sized effect was found when those participants between ages 22 and 25 were compared to those aged 30 to 34 by gender $\chi^{2}$ $(1, N=986,498)=45,975.72, p<0.001$, Cramér's $V=0.349$. A medium effect was also found when participants between 22 and 25 were compared to those aged 35 to 49 by gender $\chi^{2}(1, N=986,498)=28,416.25, p<0.001$, Cramér's $V=0.303$. Comparisons between those aged 26 to 29 and those aged 30 to 34 revealed a medium sized gender effect $\chi^{2}(1, N=986,498)=20,033.50, p<0.001$, Cramér's $V=0.338$. Those aged 26 to 29 differed significantly from those aged 50 to 64 by gender $\chi^{2}(1, N=986,498)=16,237.68$, $p<0.001$, Cramér's $V=0.391$. A small gender effect was found when participants aged 26 to 29 were compared to those aged 65 or older $\chi^{2}(1, N=986,498)=2,246.41, p<$ 0.001, Cramér's $V=0.184$. A medium sized gender effect was found when participants aged 30 to 34 were compared to those aged 35 to $49 \chi^{2}(1, N=986,498)=33,611.20, p<$ 0.001, Cramér's $V=0.306$. Participants aged 35 to 49 differed significantly by gender when compared to those aged 50 to $64 \chi^{2}(1, N=986,498)=21,596.45, p<0.001$, Cramér's $V=0.273$.

Although statistically significant differences were also found in the group who did not perceive a need for treatment, there was almost no association in Table 4. A closer examination of Table 3 reveals that there were notable differences in gender and age particularly where a larger portion of women in the 22-25 age group perceived a need for 
treatment, and men aged 30-34 and over age 50 far outnumbered the number of women who perceived a need for treatment at those ages.

Marital status. Statistically significant chi-square test statistics were found within both the perceived treatment need group and the group which did not perceive a need for treatment when gender and marital status were examined. However, Tables 3 and 4 reveal that the portion of each gender represented in each level of marital status only had a small effect for the perceived treatment need group and virtually no effect for the group which did not perceive a need for treatment. Upon reviewing Table 3, it appears as though this difference may have been identified where proportionately more married men perceived a need for treatment than married women, while more women who were widowed, divorced, or separated perceived a need for treatment than men of a similar status.

Post-hoc chi-square tests on those who perceived a need for treatment by gender and marital status revealed some significant differences. There was a small gender effect on the difference between those who were married compared to those who divorced, widowed, or separated $\chi^{2}(1, N=986,498)=33,895.76, p<0.001$, Cramér's $V=0.244$. There was also a small gender effect on the difference between those who were divorced, separated, or widowed when compared to participants who were never married $\chi^{2}(1$, $N=986,498)=18,201.03, p<0.001$, Cramér's $V=0.171$.

Education. A large proportion of both groups had received at least a high school diploma. Chi-square test statistics were statistically significant within the perceived need for treatment group, revealing that there were unequal portions of men and women who received a high school diploma and this was also confirmed with a small effect size, as 
seen in Table 3. Table 4 revealed a statistically significant difference for the group of individuals who did not perceive a need for treatment, however, a negligible effect size was found.

County. Although statistically significant chi-square test statistics were produced for both the perceived treatment need group and the group which did not perceive a need for treatment, neither resulted in effect sizes that showed the association between gender and county of residence.

Income. Table 3 presents the chi-square test of homogeneity which revealed that proportions of income were not equal across genders in the perceived need for treatment group, $\chi^{2}(3, N=986,498)=53,486.15, p<0.001$, Cramér's $V=0.233$. The test revealed that proportions for each level of income were significantly different by gender, with the effect size coefficient revealing a small to medium association between variables. A small gender effect was found to exist when comparisons were made between participants with family incomes below $\$ 20,000$ and those with incomes between $\$ 20,000$ and $\$ 49,999 \chi^{2}(1, N=986,498)=52,293.79, p<0.001$, Cramér's $V=0.273$. A smaller gender effect was found when comparing those with the lowest income and those with family incomes between $\$ 50,000$ and $\$ 74,999 \chi^{2}(1, N=986,498)=13,702.57, p<$ 0.001 , Cramér's $V=0.161$. There were significant differences by gender between participants in the lowest income bracket compared to those in the highest income bracket $\chi^{2}(1, N=986,498)=12,196.32, p<0.001$, Cramér's $V=0.168$. A small income effect was found when the gender differences between participants with family incomes from $\$ 20,000$ to $\$ 49,999$ were compared to those with incomes between $\$ 50,000$ and $\$ 74,999 \chi^{2}(1, N=986,498)=5,969.55, p<0.001$, Cramér's $V=0.104$. 
Table 4 presents the same test values for the group of participants who did not perceive a need for treatment. Proportions of the group also had unequal representations of each gender, $\chi^{2}(3, N=19,462,975), p<0.001$, Cramér's $V=0.057$ with virtually no effect between the variables.

Health insurance status. Significant gender differences were found in both groups on the variable of health insurance status. In both groups, significantly more women had health insurance than men. A medium sized gender effect was found in the group of individuals who perceived a need for treatment, where $77.5 \%$ of women had health insurance while $55.7 \%$ of men did.

Anxiety in the past year. Statistically significant chi-square test statistics were found within both the perceived treatment need group and the group which did not perceive a need for treatment when gender and past year anxiety were examined. Small gender effects were found in each group, with more women reporting having experienced anxiety in the past year than men.

Depression in the past year. Table 3 presents the chi-square test of homogeneity which revealed that the number of participants who reported depression symptoms in the past year was not equal across both genders in the perceived need for treatment group, $\chi^{2}(1, N=986,498)=40,097.95, p<0.001$, Cramér's $V=0.203$. The test revealed that experiencing depressive symptoms differed significantly by gender, with the effect size coefficient revealing a small to medium effect of gender. It appears as though roughly one third more women reported depression within the past year when compared to men Table 4 presents the same test values for the group of participants who did not perceive a need for treatment. Proportions of the group also had unequal representations of each 
gender, $\chi^{2}(1, N=19,462,975),=936,156.03, p<0.001$, Cramér's $V=0.221$ with also a small to medium gender effect. In this group, it appeared as though over three times as many women reported experiencing depression in the past year when compared to men.

Prior substance use treatment. Significant gender differences were found in both groups in terms of prior substance use treatment. In both groups, significantly more men reported receiving prior substance use treatment than women. A small gender effect was found in the group of individuals who perceived a need for treatment, with virtually no gender effect found in the group that did not perceive a need for treatment.

Reasons for not seeking treatment. Table 5 summarizes the most commonly endorsed reasons among all participants who responded to these questions. Among the top reasons for not receiving treatment were: that participants did not want to stop using their substance(s) of choice (39.5\%), participants could not afford treatment or did not have insurance to pay for it (33.4\%), participants were concerned about what their neighbors would think of them if they received treatment (13.1\%), participants believed that they could handle their substance use problem without treatment (11.0\%), and they did not have access to transportation $(10.6 \%)$.

Table 6 and 7 also summarize the top responses, however they are separated by gender and some notable gender differences can be observed. This is particularly interesting, as there were a roughly equal number of men and women who perceived a need for treatment, yet there are different portions which endorsed each of the reasons for not receiving treatment and different reasons comprised the top five for each gender. According to Table 6, the top reasons which women reported not receiving treatment 
were: not being ready to stop using substances (47.5\%), not being able to afford treatment or not having insurance to pay for it $(35.0 \%)$, believing that they could handle their substance use without treatment (15.7\%), fearing it would negatively impact their job (14.5\%), and two responses were tied for the fifth common reason. Roughly $12.8 \%$ of women reported that they did not pursue treatment because they did not have access to transportation and because they did not want others to know. Table 7 reveals that most commonly endorsed reasons for not receiving treatment among men who perceived a need for treatment in the sample. The most common reason was not being able to afford treatment or lacking insurance coverage $(31.9 \%)$, followed by not being ready to stop using substances $(31.5 \%)$, being concerned about what their neighbors would think (14.6\%), not feeling a need for treatment at the time $(9.8 \%)$, and not knowing where to go to get treatment $(8.6 \%)$. 


\section{Assumptions of Logistic Regression}

Testing for multicollinearity. Bivariate correlations were conducted to test for multicollinearity and the highest correlation between any two variables was the phi correlation coefficient value of 0.456 between past year anxiety and past year depression. No two variables were found to meet the cut-off $(r=0.90)$ as detailed in the last chapter, so there were no redundant variables impacting the model and no new composite variables needed to be created as a result.

\section{Missing Data}

As described earlier, the use of weighted data was meant to account for any unit nonresponse. Missing variable analyses were conducted in SPSS for each variable included in the model to test for item nonresponse. Only three of the included variables were missing any values and each of them was a covariate involved in the analyses for the fourth, fifth, and sixth research aims. The variables which focused on past year anxiety and past year depression were each missing $1.7 \%$ of responses, while the variable regarding past substance use treatment was missing . $3 \%$ of responses. In total, 102 participants ( $1.9 \%$ of the unweighted sample) were found to be missing any data; nine were missing a single value on a variable, 87 were missing values on two variables, and six were missing values on all three variables. These cases were excluded from analyses for the fourth, fifth research aims due to the aforementioned description of usable versus nonusable cases in the public use dataset. The cases left with remaining missing values in the public use data set were not intended to be used in analyses (RTI, 2012). 


\section{Outliers}

It was apparent that the large proportion of indicated cases being deemed as outliers was due to the fact that the vast majority of cases in the sample did not indeed perceive a need for treatment. Although the standard protocol would be to consider removing such outliers from the analyses altogether because logistic regression is sensitive to outliers, they were left in because they were most of the participants who actually perceived a need for treatment.

\section{Logistic Regression and Rare Events Logistic Regression}

Analyses were performed with perceived treatment need as the dependent variable. Standard binary logistic regressions were first conducted using SPSS and then again using rare events logistic regression in Stata in order to confirm results. Research aims and hypotheses will now be reviewed in light of statistical analyses, detailing model characteristics and evaluating hypotheses and outcomes.

Research Question 1: Is greater substance use problem severity associated with an increased likelihood that individuals who meet criteria for SUDs will perceive a need for treatment compared to lower levels of substance use problem severity? Hypothesis 1: Individuals who meet criteria for SUDs will be more likely to perceive a need for treatment at greater levels of substance use problem severity compared to lower levels of substance use problem severity. A standard logistic regression was first conducted in order to test this hypothesis and all weighted cases $(N=20,077,235)$ were included in the analysis. The model significantly predicted perceived treatment need (omnibus chi-square $=541990.11, \mathrm{df}=2, p<0.0001)$, indicating that the model was able to distinguish 
between those individuals who met criteria for SUDs who perceived a need for treatment and those who did not. It explained between $2.6 \%$ (Cox \& Snell R square) and 8.2\% (Nagelkerke R squared) of the variance in perceived treatment need, with $95.2 \%$ of predictions were accurate. Table 9 presents the coefficients and standard errors, Wald statistics and associated degrees of freedom, and odds ratios for each of the variables. The Wald criterion demonstrated that substance use problem severity made a significant contribution to prediction of perceived treatment need $(p<0.001)$. The values of the coefficients reveal that increases in substance use problem severity are associated with increased odds of perceiving a need for treatment. In particular, an increase in substance use problem severity, from a single substance abuse diagnosis (mild severity) to a single substance dependence diagnosis (moderate severity), was associated with an increase in the odds of perceiving a need for treatment by a factor of 4.139 (95\% CI 4.114-4.164). Additionally, an increase from a single substance abuse diagnosis (mild severity) to multiple substance use diagnoses (high severity) was associated with an increase in the odds of perceiving a need for treatment by a factor of 8.409 (95\% CI 8.356-8.463).

A rare events logistic regression was then conducted in order to confirm the standard logistic regression results and all weighted cases $(\mathrm{N}=20,077,235)$ were included in the analysis. The $B$ coefficients for both levels of problem severity and their associated odds ratios from the rare events logistic regression model were the same as those produced in the standard logistic regression, as was the coefficient for the constant.

For this research aim it was hypothesized that odds ratios for perceived treatment need would increase as substance use problem severity increased and the logistic regression model was consistent with this hypothesis. 
Research Question 2: Is the gender of individuals who meet criteria for SUDs associated with an increased likelihood of perceiving a need for treatment? Hypothesis 2: Women who meet criteria for SUDs will be significantly more likely to perceive a need for treatment than men who meet criteria for SUDs. A standard logistic regression was first conducted to test this hypothesis and all weighted cases $(N=20,077,235)$ were included in the analysis. The model significantly predicted perceived treatment need (omnibus chi-square $=38848.85, \mathrm{df}=1, p<0.001$ ). It explained a negligible amount of the variance in perceived need- between $0.20 \%$ (Cox \& Snell R square) and $0.60 \%$ (Nagelkerke R squared). Overall, 95.2\% of predictions were accurate. Table 10 presents the coefficients, Wald statistics and associated degrees of freedom, and odds ratios for each of the variables. The Wald criterion demonstrated that gender made a significant contribution to prediction of perceived treatment need $(p<0.001)$. The values of the coefficients reveal that women are more likely than men to perceive a need for treatment by a factor of $1.504(95 \%$ CI $1.498-1.511)$.

A rare events logistic regression was then conducted in order to confirm the standard logistic regression results and all weighted cases $(N=20,077,235)$ were included in the analysis. The $B$ coefficient for gender and its odds ratio from the rare events logistic regression model were the same as the one resulting from the standard logistic regression, as was the coefficient for the constant.

For this research aim it was hypothesized that the odds ratio would indicate that women were more likely to perceive a need for treatment than men and the logistic regression model is consistent with this hypothesis. 
Research Question 3: Does the gender of individuals who meet criteria for SUDs moderate the likelihood that they will perceive a need for treatment at greater levels of substance use problem severity? Hypothesis 3: Women who meet criteria for SUDs will be more likely than men to perceive a need for treatment at greater levels of substance use problem severity. A standard logistic regression was first conducted using the 'enter' method and variables were entered in a single block in order to test this hypothesis. Substance use problem severity, gender, and the interaction terms for substance use problem severity $\mathrm{x}$ gender were entered. Two interaction terms resulted; gender and moderate problem severity, and gender and high problem severity. All weighted cases $(N=20,077,235)$ were included in the analysis. The model significantly predicted perceived treatment need (omnibus chi-square $=574070.622, \mathrm{df}=5, p<0.0001$ ). It explained between $2.8 \%$ (Cox \& Snell R square) and 8.6\% (Nagelkerke R squared) of the variance in perceived treatment need. In sum, $95.2 \%$ of predictions were accurate. Table 11 presents the coefficients, Wald statistics and associated degrees of freedom, and odds ratios for each of the variables. The Wald criteria showed that all variables and (substance use problem severity, gender, and both interaction terms) made a significant contribution to prediction of perceived treatment need $(p<0.0001)$. The values of the coefficients reveal that women were significantly less likely than men to perceive a need for treatment by a factor of $0.707(95 \%$ CI 0.698 - 0.715) when they met criteria for moderate substance use problem severity compared to when they met criteria for mild substance use problem severity. Women were about as likely as men to perceive a need for treatment when they met criteria for high substance use problem severity compared to mild substance use problem severity by a factor of 1.028 (95\% CI $1.015-1.041)$. 
A rare events logistic regression was then conducted in order to confirm the standard logistic regression results and all weighted cases $(\mathrm{N}=20,077,235)$ were included in the analysis. The $B$ coefficient for both interaction terms and their log odds ratios from the rare events logistic regression model were the same as those resulting from the standard logistic regression, as was the coefficient for the constant.

It was hypothesized that the odds ratio would indicate that women were more likely to perceive a need for treatment than men as substance use problem severity increased. The model was not consistent with this hypothesis at either level of substance use problem severity.

Research Question 4: How do predisposing and enabling individual determinants impact the moderating influence of gender on the likelihood to perceive a need for treatment? A standard logistic regression was conducted using the 'enter' method and variables were entered in a single block so as to test this research question. The predisposing and enabling variables (race/ethnicity, age, marital status, education, county, income, past year anxiety, past year depression, and prior substance use treatment) were each entered in to the equation, followed by substance use problem severity, gender, and the interaction variables (substance use problem severity $\mathrm{x}$ gender). Table 12 summarizes model characteristics. A total of 19,981,581 weighted cases were analyzed and the full model significantly predicted perceived treatment need (omnibus chi-square $=$ 1580886.559, $\mathrm{df}=29, p<0.0001)$, indicating that the model was able to distinguish between individuals who met criteria for SUDs who perceived a need for treatment and those who did not. The model explained between 7.6\% (Cox \& Snell R square) and 
23.6\% (Nagelkerke R squared) of the variance in perceived treatment need. Overall, $95.3 \%$ of predictions were accurate.

When the interaction terms (substance use problem severity $\mathrm{x}$ gender) were added to the model to test the relationship within the context of predisposing and enabling factors, statistically significant moderation effects were found at both levels of substance use problem severity. Compared to men, women were significantly less likely to perceive a need for treatment at the moderate level of substance use problem severity than at the mild level of substance use problem severity by a factor of 0.563 (95\% CI $0.556-0.570)$ while controlling for all other predisposing and enabling factors. Women were also less likely than men to perceive a need for treatment at the high level of substance use problem severity compared to the mild level of substance use problem severity by a factor of $0.852(95 \%$ CI $0.840-0.863)$. These $B$ coefficients were also found when the rare events logistic regression was conducted.

Significant main effects for various predisposing and enabling factors were also found in the full model. Race and ethnicity were found to differently impact the likelihood to perceive a need for treatment for many participants in the sample. In this situation, non-Hispanic whites were the reference group for all analyses. It was found that Native Hawaiian Islanders and other Pacific Islanders were much more likely to perceive a need for treatment than non-Hispanic whites by a factor of $9.95(95 \%$ CI $9.77-10.13)$. Native Americans, Alaska Natives, non-Hispanic bi/multiracial individuals, and Hispanic individuals were also more likely to perceive a need for treatment than non-Hispanic whites. It was also found that Asians were the least likely to perceive a need for treatment when compared to non-Hispanic whites by a factor of 0.16 (95\% CI $0.15-0.17)$. 
Age was also significantly related to perceived treatment need. With the youngest adults as the reference group (aged 18-21), it was found that they were the least likely to perceive a need for treatment. Odds ratios for perceived treatment need increased gradually for each age bracket until hitting the highest for the individuals in the 35-49 year age group, who were more likely to perceive a need for treatment compared to the youngest adults by a factor of 3.53 (95\% CI 3.51-3.56). After this age bracket, perceived need declined again, but even adults in the 65 and older bracket were still more likely to perceive a need for treatment when compared to the youngest group by a factor of 1.50 (95\% CI 1.44-1.57).

Participants who were married appeared to be most likely to perceive a need for treatment when compared to the participants who reported either of the other two statuses. In addition, those with the highest total annual family incomes in the sample $(\$ 75,000$ or more) were the least likely to perceive a need for treatment. Participants who reported having health insurance were less likely to perceive a need for treatment compared to those who did not have health insurance by a factor of 0.64 (95\% CI $0.63-$ 0.65). Participants who experienced co-occurring anxiety or depression in the past year were also more likely to perceive a need for treatment than those who did not experience those symptoms. Also, participants who reported receiving substance use treatment before were more likely to perceive a need for treatment than those who never received treatment by a factor of 2.83 (CI 95\% 2.82-2.84).

Research Question 5: How are predisposing and enabling individual determinants and substance use problem severity associated with the likelihood of perceived treatment need among women? A standard binary logistic regression was 
conducted among only female participants in the sample. All predisposing and enabling individual determinants (race/ethnicity, age, marital status, education, county, income, health insurance status, past year anxiety, past year depression, and prior substance use treatment) were entered alongside substance use problem severity. Table 13 summarizes model characteristics. A total of $8,172,619$ weighted cases were analyzed and the full model significantly predicted perceived treatment need (omnibus chi-square $=$ 813683.25, $\mathrm{df}=26, p<0.0001$ ), indicating that the model was able to distinguish between individuals who met criteria for SUDs who perceived a need for treatment and those who did not. The model explained between 9.5\% (Cox \& Snell R square) and $26.4 \%$ (Nagelkerke R squared) of the variance in perceived treatment need. Overall, $94.2 \%$ of predictions were accurate.

Many significant main effects were found between various factors in the model. For the racial/ethnic analysis, non-Hispanic whites were the comparison group. The model revealed that non-Hispanic black women were half as likely as non-Hispanic white women to perceive a need for treatment. Meanwhile, women in almost all of the other racial/ethnic groups were in fact more likely to perceive a need for treatment compared to non-Hispanic white women. Women who identified as Native Hawaiian Islander and other Pacific Islander were over 18 times more likely than non-Hispanic white women to perceive a need for treatment. Native American and Alaska Native women were more likely to perceive a need for treatment than non-Hispanic white women by a factor of 1.21 (95\% CI 1.19-1.22). Women who identified as non-Hispanic biracial were three times more likely than non-Hispanic white women to perceive a need for treatment. 
For age-related analyses, the group of young adult women between the ages of 18 and 21 were the reference. Perceived need for treatment generally appeared to increase by age, with the largest group of women with a perceived need for treatment being between the ages of 35 and 49 years old. They were almost 5 times more likely than the young adult women to perceive a need for treatment. Women between the ages of 50 and 64 were then less likely to perceive a need for treatment when compared to the youngest women in the sample by a factor of 0.65 ( $95 \%$ CI $0.62-0.68)$.

Marital status was also significantly associated with perceived treatment need among women in the sample. The model suggests that women who reported being widowed, divorced, or separated were slightly less likely to perceive a need for treatment than married women by a factor of 0.84 (95\% CI $0.83-0.85)$. Never married women were less likely than married women to perceive a need for treatment by a factor of 0.64 (95\% CI 0.63-0.64).

Women who reported graduating high school were significantly more likely to report a perceived need for treatment than women without a high school diploma by a factor of 1.71 (95\% CI 1.69-1.72). County of residence was also related to perceived need for treatment among women. Compared to women who lived in large metropolitan areas, women in smaller towns were almost half as likely to perceive a need for treatment. However, women in non-metropolitan or rural areas were 1.24 times more likely to perceive a need for treatment than women in large metropolitan areas.

Total family income was significantly related to perceived treatment need. Women in the lowest income bracket, with a family income of below $\$ 20,000$, were most likely to perceive a need for treatment compared to women with higher family incomes. 
Women from families with over $\$ 75,000$ in total income per year were less likely to perceive a need for treatment by a factor of 0.37 ( $95 \%$ CI $0.37-0.38)$ when compared to women with family incomes below $\$ 20,000$ per year. Women who reported anxiety in the past year were more likely to perceive a need for treatment than women who did not. Women who reported depression in the past year were also more likely to perceive a need for treatment than women who did not. Women who reported that they had received substance use treatment in the past were more likely to perceive a need for treatment by a factor of 2.73 (95\% CI 2.71-2.75) compared to women who had not received treatment in the past. In addition, women who experienced moderate substance use problem severity were twice as likely as women with mild problem severity to perceive a need for treatment. Women who experienced high substance use problem severity were over seven times more likely to perceive a need for treatment than women with mild substance use problem severity.

Research Question 6: How are predisposing and enabling individual determinants and substance use problem severity associated with the likelihood of perceived treatment need among men? A standard binary logistic regression was conducted among only male participants in the sample. All predisposing and enabling individual determinants (race/ethnicity, age, marital status, education, county, income, health insurance status, past year anxiety, past year depression, and prior substance use treatment) were entered alongside substance use problem severity. Table 14 summarizes model characteristics. A total of 11,904,617 weighted cases were analyzed and the full model significantly predicted perceived treatment need (omnibus chi-square $=$ 945545.75, $\mathrm{df}=26, p<0.0001$ ), indicating that the model was able to distinguish 
between individuals who met criteria for SUDs who perceived a need for treatment and those who did not. The model explained between 7.6\% (Cox \& Snell R square) and $26.2 \%$ (Nagelkerke R squared) of the variance in perceived treatment need. Overall, $96.2 \%$ of predictions were accurate.

A number of significant main effects were found in the model. Men who identified as non-Hispanic black, Native American, and Alaska Native were more likely to perceive a need for treatment than non-Hispanic white men. Meanwhile, men who identified as Asian and men who identified as non-Hispanic bi/multiracial were less likely to perceive a need for treatment compared to non-Hispanic white men. Men in age groups over 30 were more likely to perceive a need for treatment when compared to the men in the youngest age bracket (18-21). Men between 35 and 49 years of age were more likely to perceive a need for treatment by a factor of 3.15 (95\% CI 3.12-3.18) when compared to men between the ages of 18 and 21. Men between 50 and 64 were almost three and a half times more likely to perceive a need for treatment when compared to the youngest men in the sample.

Married men were most likely to perceive a need for treatment when compared to men who were widowed, divorced, separated, or never married. Men who graduated high school were less likely to perceive a need for treatment than men who did not by a factor of 0.68 (95\% CI $0.67-0.68)$. Men with family incomes between $\$ 20,000$ and $\$ 49,999$ in the past year were almost twice as likely to perceive a need for treatment when compared to men in the lowest bracket (earning below $\$ 20,000$ in the past year), and men in families with a total income between $\$ 50,000$ and $\$ 74,999$ were more likely to perceive a need for treatment than men in the lowest income bracket by a factor of 2.24 (95\% CI 
2.22-2.27). Men in the highest income bracket (family income over $\$ 75,000$ in the past year) had a comparable likelihood of perceiving a need for treatment than men in the lowest income bracket. Results indicate that having insurance is associated with being less likely to perceive a need for treatment than being without insurance. Men who reported having anxiety in the past year were two and a half times more likely to perceive a need for treatment than men who did not. Similarly, men who experienced depression in the past year were also two and a half times more likely to perceive a need for treatment than those did not. Men who reported prior substance use treatment were over three times more likely to perceive a need for treatment compared to men who had never received substance use treatment. Men who experienced moderate substance use problem severity were a little over three times more likely to perceive a need for treatment compared to men with mild substance use problem severity. Men who experienced high substance use problem severity were more likely to perceive a need for treatment than men with mild substance use problem severity by a factor of 5.54 (95\% CI 5.48-5.59). 


\section{CHAPTER V. DISCUSSION}

This study was conducted in an effort to fill gaps in the existing literature in several ways: to examine the impact of varied substance use problem severity upon the perceived treatment need among individuals who met diagnostic criteria for SUDs, to determine whether female gender had a moderating impact upon that relationship, and to explore whether predisposing and enabling factors were differently associated with perceived treatment need between women and men. Given that these questions had never been explored in the relevant literature regarding this issue, and especially with such a large and diverse sample, results of this study make a meaningful contribution to this knowledge base. This study integrated variables from the substance use literature and Andersen and Newman's health service utilization model (1973/2005) to examine the impact of gender while taking into account the role of substance use problem severity and various predisposing and enabling factors on perceived need for treatment. Thus, understanding the connections between problem severity and gender have been enhanced, as well as the gender differences in perceived treatment need, providing new opportunities for outreach, assessment, and intervention efforts.

This chapter discusses and interprets the results of this study. First, a summary of

all major findings will be presented. This will be followed by a more thorough analysis of each research question and the associations found between the variables. The results will then be examined in light of the fact that the vast majority of the sample did not perceive a need for treatment despite meeting diagnostic criteria for SUDs. This discrepancy between expert-defined need for treatment and user-defined treatment need will be discussed, as well as the fact that the analyses are focused upon such a small portion of 
the sample. Then suggestions for future studies in this area will be presented, along with limitations of the current study, and implications for social work and public health professionals.

\section{Sample Characteristics}

Males comprised the majority of the final weighted sample (59.6\%); however this was not a significant gender difference. As noted in the literature review, the gender gap in SUDs has been closing over the years and, in fact, the rate of SUDs among women compared to men is higher in this study than reported in the 2009 NSDUH (SAMSA, 2010b). There was almost a perfect 50:50 gender split in the perceived treatment need group, whereas there was a 60:40 male to female ratio amongst individuals who did not perceive a need for treatment.

Substance use problem severity levels were not equally distributed within the sample, as most participants fell into the mild category and the fewest fell into the high severity category. There were also noteworthy differences between groups, with individuals in the perceived treatment need group having higher proportions of moderate and high severity substance use problems and the group who did not perceive a need for treatment had higher proportions of individuals with low and moderate substance use problem severity. Although there was a great degree of overlap between groups, this was still an indicator that problem severity was related to likelihood of perceiving a need for treatment and was later confirmed with the logistic regression analyses.

The study sample was very racially and ethnically diverse and represented all major groups, and a number of minority groups, who currently live in the U.S. This was 
one of the main strengths of this study which made it distinct from other studies on perceived treatment need because almost half of the sample consisted of minority groups. The racial and ethnic breakdown of this sample can be compared to actual 2010 census data taken the same year of the survey to see if these racial and ethnic groups proportionately met criteria for SUDs (Humes, Jones \& Ramirez, 2011). Proportions were generally close, with some notable differences. It appears as though non-Hispanic whites were $76.2 \%$ of the US population in the year of the survey, while they comprised $63.5 \%$ of the individuals with SUDs. Non-Hispanic blacks comprised a little over $10 \%$ of individuals with SUDs, yet were actually $14.6 \%$ of the general population at the time. Asians were also underrepresented in the sample compared to their presence in the general population. However, Native Americans and Alaska Natives, Native Hawaiian Islanders and other Pacific Islanders, and non-Hispanic biracial/multiracial people were overrepresented among individuals with SUDs and actually comprised a smaller percentage in the national population. Hispanic-identifying individuals were the only group proportionately represented in the sample.

The inclusion of individuals of different backgrounds is important to better understand how racial/ethnic identity can impact the perception of treatment need. Review of the main effects of race and ethnicity from the logistic regression revealed that non-white minority status generally led to a greater likelihood of perceiving a need when compared to non-Hispanic whites. There were only two notable exceptions to this, with non-Hispanic blacks appearing to be equally likely as non-Hispanic whites to perceive a need for treatment, and for Asians to be dramatically less likely to perceive a need for 
treatment compared to non-Hispanic whites. Gender differences in this area were also found when research aims 5 and 6 were explored and this will be detailed later.

These findings are much richer than in previous studies which have examined racial and ethnic background and perceived treatment need because a larger number of ethnic groups were included in the analysis and their numbers were more substantial. Noting that many minority ethnic and racial groups are more likely to perceive a need for treatment than non-Hispanic whites is important, as most of these minorities are generally underrepresented in treatment settings. Better understanding why the discrepancy exists between them perceiving a need for treatment and not receiving that treatment could lead to more targeted efforts at addressing these potentially racially- and/or ethnically-specific barriers.

There were a large number of participants in the study who were under the age of 26 due to the sampling strategy and aims of the NSDUH itself as noted earlier. In spite of this, notable age-related differences were still found in perceived treatment need. The likelihood of perceiving a need for treatment appeared to be higher with older participants, with the highest likelihood within the 35-49 year old bracket and then decreasing again by the oldest cohort of participants over the age of 65 . These findings are consistent with several prior studies on perceived treatment need which found that young adults are less likely to perceive a need for treatment (Grella et al., 2009; Wu \& Ringwalt, 2004). As noted earlier, epidemiological studies suggest that younger and more recent cohorts are more likely to have experimented with substances and have more accepting views of substance use in general. They are also more likely to be susceptible engage in behaviors based on their perceptions of what is the 'norm' among their peers, 
which can explain why they are less likely to not label certain degrees of use as problematic (O'Callaghan, Chant, Callan \& Baglioni, 1997). This may potentially explain why younger cohorts are less likely to perceive a need for treatment, as their use may be comparable of that of their peers.

The overrepresentation of young adults could also explain why over half of the overall sample consisted of never married individuals. Interestingly, being married was found to increase the likelihood that an individual would perceive a need for treatment compared to the other two statuses. And this relationship held even in the gender-specific analyses. It is possible that a spouse can increase your awareness that your substance use is becoming a problem or affecting your ability to manage responsibilities.

In particular, the largest portion of participants who were married was actually among the men who perceived a need for treatment. This finding is confirmed by some literature which shows that, for example, alcoholic men are less likely to be married to partners who drink than women (Walitzer \& Dearing, 2006). (This finding about marriage is likely to translate to illicit substance users as well, given that the majority of illicit substance users are male and this may make it more likely for them to be married to women who do not use those substances.)

The vast majority of the sample (80.9\%) consisted of participants who graduated from high school and this factor did not appear to have a notable association with perceived treatment need (Table 12). One interesting finding, based on a review of proportions in each group revealed that only $66 \%$ of men who perceived a need for treatment had a high school diploma. The gender-specific logistic regression models also found notable differences in education being associated with perceived treatment need. 
In addition, over half of all participants were living in households where the total income was $\$ 49,999$ per year or less. Income was associated with the likelihood of perceiving a need for treatment among participants in the sample, illustrated by those with the highest incomes being least likely to perceive treatment (see Table 12). It could be that these participants may be more likely to be insulated from the potential negative effects of their substance use due to having more resources. According to Andersen and Newman's model (1973/2005), it may also be that having higher incomes enabled them to seek out and to be able to afford treatment when they wanted it.

Almost three quarters of all participants reported having health insurance coverage at the time of the survey (see Table 1). The proportion of individuals with health insurance was lower in the group that perceived a need for treatment, with $66.5 \%$ of them having health insurance. This link was also demonstrated when cost/no insurance was listed as the second most common reason that treatment was not received by those who perceived a need for treatment. Upon closer examination, a significant gender difference was found where a higher proportion of women who perceived a need for treatment (77.5\%) reported having health insurance within the past year than men who perceived a need for treatment (55.7\%) (see Table 3). This reveals an otherwise unexplored and unidentified gender difference. This finding suggests that not having health insurance may be a larger barrier to pursuing treatment for men who perceive a need for treatment than for women who do.

There were almost equal rates of reported anxiety and depression in the past year among all participants in the sample, at $12.5 \%$ and $12.7 \%$ respectfully. However, proportions of those in each group were dramatically different, which reveals why these 
disorders were shown to increase the likelihood of perceiving a need for treatment. In fact, experiencing anxiety in the past year increased the likelihood of perceiving a need for treatment by a factor of $2.15(95 \%$ CI $2.14-2.17)$ and experiencing depression in the past year increased the likelihood by a factor of 1.63 (95\% CI $1.62-1.64)$. This can also be seen by reviewing proportions in the groups. Between 11 and $12 \%$ of the participants who did not perceive a need for substance use treatment experienced anxiety or depression in the past year. In contrast, 35 to $37 \%$ of the individuals who perceived a need for treatment reported experiencing either depression or anxiety. This confirms findings in the literature cited earlier as well, which suggest that co-occurring substance use disorders and mental health diagnoses increase perceived treatment need. What is interesting here is that, despite having separate mental health problems, these participants still reported perceiving a need for substance use treatment in particular. This had not been able to be teased apart in some other studies which often lumped substance use treatment and mental health treatment together when asking about perceived treatment need.

Roughly one-fifth of the sample reported having substance use treatment in the past which is relatively high for general populations; however this sample consists of only of substance users which may explain this. A review of the proportions in each group shows that over half of all individuals who perceived a need for treatment had reported being in substance use treatment before. Among those who did not perceive a need for treatment, approximately $20 \%$ received treatment in the past. This is a relatively large difference. The logistic regression analyses confirmed that prior substance use 
treatment was associated with increased the likelihood of perceiving a need for treatment as well.

\section{The rarity of perceived treatment need}

The results of this study are comparable to others which also found that the large majority of individuals who meet the criteria for SUDs do not, in fact, perceive a need for treatment (Grella et al., 2009; Mojtabai et al., 2002; Ryan et al., 1995; SAMHSA, 2010b; Tucker \& Simpson, 2011; Tucker et al., 2011; Wu \& Ringwalt, 2004). In this weighted sample of over 20 million Americans who met diagnostic criteria for SUDs, a mere 4.8\% $(986,498)$ indicated that they felt that they needed treatment for their use in the past year. This rate is consistent with NSDUH results since 2006 which have shown average rates of perceived treatment need to be around 5.1\% among participants who met diagnostic criteria for SUDs (SAMHSA, 2010b). As noted earlier, SAMHSA and other treatment professionals would determine that this entire study's sample would be in "need" of treatment due to meeting diagnostic criteria for SUDs yet there is an obvious discrepancy between each party's perceptions of the situation. Referring back to the TTM, the vast majority of the sample of individuals who met criteria for SUDs would likely fall under the precontemplative category, with a small minority being contemplative of their substance use and its related problems.

\section{Reasons for not seeking treatment}

Among all participants who reported perceiving a need for treatment, the most commonly endorsed reason for not seeking treatment was not being ready to stop using 
substances. This is a revealing and interesting finding, as it suggests that the belief that most treatment is abstinence-based or that quitting was necessary in order to begin treatment was a large deterrent to seeking treatment. It is also noteworthy that almost half of all women (47.5\%) who reported perceiving a need for treatment endorsed the statement that having to stop using substances was why they did not pursue the treatment that they otherwise thought they needed. As noted in the literature review, there is ample evidence that ongoing heavy substance use can have detrimental effects particularly on the health and well-being of women. It is possible that lower thresholds for entering services such as non-abstinence could welcome the majority of women who are ambivalent regarding abstinence, as well as the many men who feel similarly. Upon entering treatment, these individuals may at least feel welcome to begin exploring their attitudes towards behavior change and work towards achievable goals. It is also possible that these individuals may eventually choose to pursue abstinence after starting treatment. According to the Transtheoretical Model of Change (TTM), these individuals at the contemplative stage are ambivalent about treatment because they have not yet decided on abstinence as a goal. They can still be reached and should be engaged in care. Research on TTM shows that treatment matched to motivational stage of change can help improve outcomes of clients and so individuals who perceive a need for treatment but who may not be ready for abstinence could still be assisted and engaged in steps towards change (Norcross et al., 2011).

The abstinence-only expectation is not necessarily true of all available treatment for substance use and this misconception is keeping some individuals with substance use disorders out of treatment which could help them to at least better manage their substance 
use and its associated consequences. While it is generally true that some treatment modalities require complete abstinence from substances, such as detoxification centers in hospitals and some rehabilitation centers, there are facilities which do provide harm reduction or moderation services. Rates on the availability of these types of facilities are difficult to find though, and it is likely true that most facilities require abstinence. The closest one could get to understanding the approaches used in the treatment facilities in the U.S. would be to review data from the National Survey of Substance Abuse Treatment Services (N-SSATS), an annual census of all treatment facilities in the country (SAMHSA, 2012a). According to their review, the most commonly used approach among over $94 \%$ of all treatment facilities is "substance abuse counseling," followed by relapse prevention by $84 \%$ of facilities, and twelve-step facilitation by $77 \%$ of facilities. The category of substance abuse counseling is albeit vague classification which does not provide any information as to the requirements of treatment. One could interpret that relapse prevention and twelve-step facilitation are predominantly abstinence-oriented, but it is unclear. This survey data, although based on a small subsample, does suggest that more agencies should consider lower thresholds for treatment entry to see if it could increase enrollment and engagement in services.

The second most common reason for not pursuing treatment among the sample was not being able to afford treatment or having insurance to pay for it. The cost of treatment as a deterrent for people who perceive a need for treatment is unfortunate. This is both a commentary on the potentially inhibitive costs of treatment for Americans who have economic challenges, but also the limitations of insurance coverage. This is also not unique to the field of substance use treatment alone, because many Americans struggle to 
afford basic medical care and mental health care as well. This has been written about extensively, as those who end up seeking treatment are those with the most severe problems which could have been addressed preventatively or before problems escalated greatly. This prohibitive effect of cost is also aligned with the Andersen and Newman's model (1973/2005) which clearly suggested that income, insurance, and the price of services can be enabling factors to increase the likelihood of service utilization, but that they can also prevent people for pursuing needed treatment as well. This is an issue which can and should be addressed by social workers both at the mezzo and macro level. At the mezzo level, social workers should advocate for treatment agencies to create flexible and affordable self-pay plans for individuals who are uninsured and seeking treatment. At the macro level, social workers should advocate for greater parity in substance use treatment coverage among insurance plans, as well as continuing to support the Affordable Care Act, which will extend insurance coverage to more Americans.

Beyond the top two reasons for not receiving treatment, a number of notable gender differences were found. For example, the third most commonly reported reason for not receiving treatment among men was concern about what their neighbors would think, the fourth reason being that they did not think they needed treatment at the time, and the fifth being that they were not sure of treatment agency options. A closer examination of the underlying reason of number three would reveal that the third most common reason that men did not receive substance use treatment was, essentially, due to stigma. The concern that their neighbors would look down upon them for pursuing treatment is likely due to their awareness of the stigma of having a substance use problem, as opposed to any other health condition. Surveys of public attitudes towards 
individuals with addictions confirm this, as people with addictions are viewed as "more dangerous and blameworthy" (p. 139) when compared to individuals with mental illness or physical disabilities (Corrigan, Kuwabara, \& O’Shaughnessy, 2009). Societal stigma is a large issue for any one group or profession to tackle, but efforts can and should be made. Social workers and public health workers can work on large awareness-raising anti-stigma campaigns. In addition, social work and public health training should involve opportunities for students to examine stigmatizing attitudes they hold and how they may impact their work as future professionals.

The fourth most common reason among men was that they did not pursue treatment in the past year was because they did not believe that they needed treatment at the time. Interpreted through the lens of TTM, these participants appear to have had an awareness that they were precontemplative earlier in the year about their substance use problem and after the fact, at the time of the survey; appear to have the insight into the fact that they probably did have a problem earlier. It is unclear what may have motivated this increased awareness, but social workers and public health workers have the power to help increase awareness among their clients through increased screening and informing clients how they can identify when their use may be becoming problematic. This can even be done on a larger scale through media and social media campaigns among the general population.

The fifth most common reason among men to not receive treatment was that they did not know where to go to find treatment. This could be tied to what Andersen \& Newman's model referred to as a community-level enabling factor, in terms of access to facilities. Not knowing where to go in one's community can be a deterrent for those who 
could otherwise benefit from services. In rural communities, there may not be many nearby or local options available, which could be why people are less likely to know where to access services. In addition, it is unclear how much advertising that treatment agencies do or whether they distribute promotional materials in places where potential clients may see them. This is an area for further research and exploration.

Among women, the third most commonly reported reason for not receiving treatment was believing that they could handle their problem without treatment; the fourth reason was fearing that it would have a negative impact on their employment, and the fifth was not having appropriate access to transportation in order to attend treatment.

The fact that one in six women who perceived a need for treatment yet thought they could handle their problem can be interpreted in a number of ways, given that we do not have further follow-up information. It could be that this is a sign that these women are aware that their substance use would generally indicate a need for treatment but that they would like to first try to address the problem independently. It also could be that this statement indicates a distrust of treatment or a belief that treatment may not be as effective as addressing it independently or through other supportive avenues. Only further research on this issue, perhaps even qualitative research, could help to uncover what this may mean.

Fearing a negative impact on their jobs could be related to a fear of stigma from coworkers or colleagues, if anyone from work were to find out. Perhaps these women fear that it may impact how their colleagues view their abilities and competence in the workplace. It also could be that these women believe that the time commitment of treatment could, in some way, impact their ability to meet job responsibilities and 
requirements. These types of concerns could be best managed through human resources departments or employee assistance programs (EAPs). However, among women who do not work in settings where these supports are available, this is likely a valid concern.

The fifth most common reason was related to a lack of transportation. This is a barrier which has been discussed in the literature around women and substance use treatment, and in the literature review. This could also be viewed as an enabling or inhibiting factor as well, especially if women live in rural or suburban communities where access to even public transportation is limited or inconvenient. Transportation is also associated with regular costs which may impact lower income women. An area where social workers can affect change is through the promotion of travel vouchers in their agencies, which can provide clients with bus passes for roundtrips to and from services or taxi fare. In addition, the increased use of technology, such as computer-based interventions, therapy provided via the internet, social media, text messaging, and other means can help make treatment more accessible to clients in remote places.

\section{Analysis of Dissertation Findings}

The present study examined the influence of substance use problem severity upon the perceived need for treatment among community based substance users and whether gender had a moderating relationship on that relationship. It also examined the ways in which various predisposing and enabling factors can differently impact men and women and their perceived need for treatment. Six research questions were posed regarding perceived treatment need. It was hypothesized that individuals who met criteria for SUDs would be more likely to perceive a need for treatment as their substance use problem 
severity increased. There was support for this hypothesis when a statistically significant main effect for substance use problem severity was found. Next, it was hypothesized that women who met diagnostic criteria for SUDs would be significantly more likely to perceive a need for treatment than those who were men. There was also support for this hypothesis when a statistically significant main effect for female gender was found. In addition, it was hypothesized that women who met diagnostic criteria for SUDs would be more likely than men to perceive a need for treatment as substance use problem severity increased. There was no support for this hypothesis; in fact, the interaction effect showed that, at moderate substance use problem severity, men were more likely than women to perceive a need for treatment than at the mild level of substance use problem severity. No noteworthy gender differences were found at the high level of substance use problem severity. Lastly, three additional research questions were posed. The influence of predisposing and enabling factors on the relationship between gender and perceived treatment was explored. After including these other factors in the model, men were still more likely to perceive a need for treatment than women at both moderate and high levels of substance use problem severity. A number of covariate effects were found, revealing that race/ethnicity was associated with perceived treatment need, as were income, age, marital status, health insurance status, prior substance use treatment, and experience either anxiety or depression in the past year. The final two research questions aimed to understand whether these factors had different associations for men than women. Notable gender differences were found on almost every variable included in the analysis and the implications for this are discussed later in this section. 


\section{Substance use problem severity and perceived treatment need.}

For the purpose of this study, substance use problem severity was divided into three categories: low severity, moderate severity, and high severity. Those in the low substance use problem severity category were participants who met criteria for an abuse diagnosis for a single substance, those in the moderate category met criteria for a dependence diagnosis for a single substance, and those in the high category met criteria for multiple diagnoses for multiple substances. This is a distinction in problem severity that has not yet been explored in the literature to date, although some of the research cited in the literature review came close. According to the review of the literature, more mental health diagnoses (substance-related or otherwise) generally contributed to an increase in perceived need for treatment however the increasing severity of substance-related problems was not quantified.

In the present study, participants with moderate substance use problem severity were roughly four times more likely to perceive a need for treatment compared to those with low severity substance use problems. Those with high severity substance use problems were almost eight and a half times more likely to perceive a need for treatment compared to those with low severity problems. This is a particularly noteworthy finding as it suggests that some individuals who have more severe problems with multiple substances do have a degree of insight into their problems. Acknowledging that they felt the need for treatment within the past year implies that these individuals were more aware of the negative consequences associated with their use and believed that treatment may be the way to alleviate these problems. 
This finding could be seen as understandable, considering that meeting abuse criteria is indeed quite different than meeting criteria for dependence or multiple SUDs. When individuals with abuse diagnoses are lumped together with individuals who have more severe diagnoses or multiple diagnoses, their otherwise justifiable differences in perception are not acknowledged. It is easier to meet criteria for a substance abuse diagnosis by having at least one of four substance-related problems within a year. Many recreational or casual users often blur the line into meeting abuse diagnostic criteria through risky substance use and may not see that their use indicates a need for formalized treatment because it very well may not. Research suggests that individuals who engage in risky substance use who do not meet criteria for substance dependence can often respond quite positively to interventions which are not delivered in formalized substance use treatment facilities. For example, problem drinkers who meet criteria for alcohol abuse have been found to respond to screening and brief interventions by health care providers (Babor \& Higgens-Biddle, 2000), computer- or web-based interventions (Carey et al., 2009; Hester, Delaney, \& Campbell; Pemberton et al., 2011), and also brief cognitivebehavioral therapy (Sobell \& Sobell, 2005). Promising outcomes have been also been found for drug abuse using brief interventions (Stein et al., 2008) as well as computerbased interventions (Ondersma, Svikis \& Schuster, 2007).

Diagnostic criteria for substance dependence are more likely to capture individuals with more severe substance-related problems because they set the bar higher and require several more regular negative consequences within the past year. Individuals in this category may be more likely to recognize these repeated ongoing events as being indicative that they could benefit from treatment. It is also individuals in these categories 
who are more likely to benefit from various degrees of treatment intensity, ranging from hospitalization for stabilization due to physiological dependence (particularly for alcohol, benzodiazepines, and opioids) to outpatient treatment modalities. These individuals are generally more likely to have other co-occurring problems which may require assistance or treatment as well.

The examination of substance use in this manner holds promise for a wide array of future studies in the area of substance use, as lumping together various degrees of SUDs (along with multiple SUDs) does not accurately recognize the differences between them.

\section{Effect of gender on perceived treatment need.}

Findings from this study indicated that there was a main effect of female gender upon perceived treatment need so that women were 1.5 times more likely than men to perceive a need for substance use treatment. This finding adds to the body of literature in this area which suggested that women with SUDs were more likely than men with SUDs to perceive a need for substance-related treatment (Edlund et al., 2006; Grella et al., 2011; Mojtabai et al., 2002). Unlike prior studies which tried to examine this relationship, this study is distinguished by its very recently collected data from a large nationally representative sample using the DSM-IV criteria for SUDs. However, this finding is limited by the fact that it does not control for other factors which may contribute to this gender difference, such as co-occurring mental health concerns, cultural factors, or other socioeconomic factors. Still, a main effect of gender is worth documenting, especially as its effect changes after other factors are included in the model. 


\section{Interaction effect upon perceived treatment need.}

Study findings suggest an unanticipated interaction between substance use

problem severity and gender upon perceived need for treatment at both levels of problem severity. According to analyses, men in the sample were actually $30 \%$ more likely than women to perceive a need for treatment at the moderate level compared to at the mild level of problem severity. At the high level of substance use problem severity, there are no notable gender differences in perceived need for treatment when compared to the low level of problem severity. This is a more nuanced finding than has been found in the literature, because no previous studies examined the role of different levels of problem severity upon perceived treatment need when combined with a moderation hypothesis involving gender.

It suggests that while both men and women are more likely to interpret a substance dependence diagnosis as requiring treatment than a substance abuse diagnosis, men are slightly more likely to do so. Prior research in this area found that, without accounting for substance use problem severity, women were equally or more likely than men to perceive a need for treatment (Edlund et al., 2006; Grella et al., 2009; Grella et al., 2011; Mojtabai et al., 2002. This finding suggests that, if perceived treatment need is interpreted as an indicator for unmet treatment need, that men with single substance dependence diagnoses are slightly more likely than women with substance dependence diagnoses to have an unmet treatment need. It is possible that, despite perceived treatment need, men with substance dependence diagnoses may face some more barriers to accessing treatment than women with similar diagnoses that are preventing them from receiving the treatment they believe they need. Individuals who meet criteria for 
substance dependence diagnoses are experiencing problems in their lives along at least three or more areas, which can be quite debilitating and could be addressed if voluntarily engaging in treatment for their problems. It is possible that financial barriers affect men with substance dependence more than women, leading them to be more likely to have an unmet treatment need. The evidence for this could be seen in Table 3 which suggests that significantly fewer men than women who perceive a need for treatment reported having insurance coverage during the past year. These findings, when combined with the results of Table 7 show that cost and insurance coverage are common deterrents for men.

\section{Interaction effect while including predisposing and enabling factors.}

After including the theoretically predisposing and enabling factors (Andersen \& Newman, 1973/2005) for perceived treatment need, the gender moderation hypothesis was tested again. Again, the hypothesis was not supported. Instead, the inclusion of additional factors led to a more pronounced gender difference in which men were more likely than women to perceive a need for treatment at both higher levels of substance use problem severity compared to the lowest level of problem severity. Men were almost $50 \%$ more likely at the moderate level of severity to perceive a need for treatment compared to the lowest level. There was a significant, but small difference at the highest level of problem severity, with men being approximately $15 \%$ more likely to perceive a need for treatment. The inclusion of these other factors made the gender effect more pronounced. This suggests that men have slightly higher rates of unmet treatment need when increased substance use problem severity is taken into consideration. Outside of predisposing and enabling factors, this suggests that men may face other barriers which 
still prevent them from receiving the treatment that they otherwise think that they need. These barriers may be more social or cultural because the economic and communitybased enabling factors have been controlled for in the model.

There is a growing body of literature which suggests that the social construction of gender impacts health, and that the construction of masculinity can undermine many of men's health-related attitudes, behaviors, and outcomes (Courtenay, 2000s; 2000b; Mansfield et al., 2003; Neighbors \& Howard, 1987; Smith, Tran \& Thompson, 2008). Courtenay's relational theory of gender and men's health (2000b) may provide some additional information with which to interpret this study's findings, particularly as they relate to men living in America. Courtenay's theory integrates aspects of both social constructionism and feminism to understand the gender differences in health in America today. He highlights the noteworthy characteristics of hegemonic masculinity in American culture as being demonstrated by American men in several ways, such as "the denial of weakness or vulnerability, emotional and physical control, the appearance of being strong and robust, [and] dismissal of any need for help" (Courtenay, 2000b, p.1389). He also suggests that the construction and maintenance of males as the "stronger sex" (p. 1385) requires them to continue to maintain and assert their power by engaging in behaviors which confirm the above-mentioned traits-- behaviors which frequently can put their health and wellbeing at risk. Examples of the influence of masculinity upon health include: choosing a physically strenuous and dangerous career, delaying or avoiding medical treatment for physical injuries, overestimating one's physical health, or underestimating one's risks of developing health problems (Courtenay, 2000a). A man who admits to illness, who reduces his risk behaviors, or who asks for help could be 
viewed as compromising his masculinity and his power- thereby being feminized. This study's findings could be informed by Courtenay's theory, because despite these men privately admitting in a survey that they believe that they need treatment, they may feel that they could be compromising their masculinity by actually seeking the help.

Other notable effects were found in the full model when predisposing and enabling factors were included. Asian participants in the sample were far less likely than non-Hispanic white participants to perceive a need for treatment of their substance use problems. This could also explain why they are so underrepresented in the treatment populations as well. Research suggests that because of more private, family-oriented cultures among Asians that seeking outside help for the stigmatized behavior of substance use could be viewed as bringing shame or disrepute onto the family unit (Yu, Clark, Chandra, Dias \& Lai, 2009). In addition, they may experience language barriers which are more difficult to overcome in traditional settings which may be more likely to have a bilingual Spanish-speaking staff person than an Asian language. Different levels of assimilation in general, may also lead to different understandings of formal western treatment facilities for substance use. However, research suggests that when culturallyspecific and sensitive treatment options are provided for Asian populations, they are more likely to attend and succeed in treatment (Yu et al., 2009).

Participants who identified as non-Hispanic black were the only group equally as likely as non-Hispanic white participants to perceive a need for treatment, while all other remaining racial and ethnic group were more likely to perceive a need for treatment by varying degrees. This is important, as these other ethnic groups are otherwise underrepresented in treatment settings. The higher rates of perceived treatment need 
among these other minority populations may then be understood within the context that members of these other groups may experience more barriers to care, such as issues with accessibility, culture, or stigma. These are very important areas of intervention for social workers and professionals in public health, in order to increase access to services and potentially reduce health disparities which may be a result of inadequate treatment for these groups. Individuals of some racial and ethnic minorities may experience unique cultural barriers to pursuing treatment they otherwise think they need, such as issues with perceived cultural sensitivity at treatment facilities or inadequate representation of their own minority groups at the treatment agencies. Native Hawaiian Islanders and Pacific Islanders were almost 10 times more likely to perceive a need for treatment than nonHispanic whites. Native Americans and Alaska Natives were almost twice as likely to perceive a need for treatment compared to non-Hispanic whites. Participants who identified as Hispanic or who were non-Hispanic biracial/multiracial were $27-50 \%$ more likely to perceive a need for treatment compared to non-Hispanic whites. No other studies in this area have identified these racial/ethnic trends in perceived treatment need.

An interesting trend was found in the relationship between age and perceived treatment need, also making the findings of this study unique. The youngest age group was the least likely to perceive a need for treatment and treatment need was higher and gradually increased for each subsequent age group and peaked among participants aged 35-49. After this age, the association with perceived treatment need declined with age, but even the oldest participants over 65 were still more likely to perceive a need for treatment than the youngest cohort. Compared to the youngest group of participants aged 18 to 21 , participants in their 20 s were slightly more likely to perceive a need for 
treatment. Participants in their early 30 s were over two and a half times more likely to perceive a need for treatment than the youngest participants. Participants aged 35 to 49 were three and a half times more likely to perceive a need for treatment than participants aged 18 to 21. Participants who were 50 to 64 years of age were over two and a half times more likely to perceive a need for treatment compared to the youngest participants and those over the age of 65 were $50 \%$ more likely to perceive a need for treatment. There could be a number of reasons to explain this. As noted earlier, younger cohorts of Americans have more liberal attitudes towards substance use and it has been normalized among members of this age group so that substance use is less likely to be seen as problematic enough to require treatment. In addition, perceived treatment need may be more likely among older individuals who have begun to recognize that their problematic substance use has now carried over into their 30s where they are seeing its effects on their ability to address other life responsibilities. The greater perceived need for treatment among older individuals may be due to the fact that they have been using substances for longer and are recognizing its detrimental effects.

Another unique finding of this study was that individuals who were married were significantly more likely to perceive a need for treatment than those were not. The impact of the relationship had not been explored much in the literature and no effects had been found, but marital status did have theoretical backing in the model used to develop the study. Another noteworthy finding was that perceived treatment need was slightly higher with higher income among participants in the sample, but was dramatically lower among participants in the wealthiest households. It is possible that these individuals may not see 
the financial effects of their substance use and thereby interpret it of needing formal treatment.

No prior study in this are found an association between health insurance status and perceived treatment need. It was found that having health insurance in the past year was associated with being $40 \%$ less likely to perceive a need for treatment. This could be explained by the fact that, because this is theoretically an enabling factor for receiving services, those who have insurance and wanted treatment already received it. Therefore, those who perceive a need for treatment but do not have insurance are unable to access treatment.

The research literature almost unanimously suggested that having a co-occurring mental health problem was associated with an increased likelihood of perceiving a need for treatment, although some studies were less clear on whether the perceived need was for substance use treatment specifically. This study examined that specific aspect. Participants who reported anxiety in the last year were over twice as likely to perceive a need for treatment as those who did not. Participants who reported depression in the past year were $63 \%$ more likely to perceive a need for treatment than those who did not. It could be that participants interpret these mental health problems as being somehow related to their substance use problems, so that treatment may be seen as a way to alleviate symptoms. Co-occurring mental health problems are increasingly being treated at substance use treatment facilities and it may be that promoting this information may lead more individuals who experience multiple problems to seek treatment.

Prior substance use treatment increased the likelihood of perceiving a need for treatment by a factor of 2.83 . This is noteworthy, as these individuals continue to view 
treatment as a good option for helping them to address their substance use. However, perhaps these participants did not return because they may not want to undergo the experience again or they do not want to feel as though they failed. It would be an interesting area for future research to understand why this may occur.

The model of relationships between the variables is still an important one to consider, despite the unexpected outcomes. Gender was indeed a moderator between problem severity and perceived treatment need, however instead of female gender being associated with an increased likelihood of perceiving a need for treatment, male gender was. This suggests that men with high levels of substance use problem severity are more likely to perceive a need for treatment than women and this is important information, as high severity users should be receiving the care they believe they need. Knowing that this group is comprised of slightly more men than women can provide information for outreach messages to this high risk population.

\section{Impact of predisposing and enabling factors on women}

Some unique associations between predisposing and enabling factors were found among women that have not yet been found in the literature. Whereas in the general sample non-Hispanic blacks and whites had comparable likelihoods of perceiving a need for treatment, gender-specific analyses revealed that non-Hispanic black women are $56 \%$ less likely to perceive a need for treatment than non-Hispanic white women. This could be an interesting area of future research, as black women are underrepresented in most treatment settings. Native Hawaiian Islander and Pacific Islander women were 18 times more likely to perceive a need for treatment than non-Hispanic white women. The fact 
that these women are underrepresented in treatment reveals that specific obstacles restrict them from receiving it. This has been documented in the literature among women in this ethnic group who are subject to stigma, family responsibilities, and other difficulties with gaining access to services (Ta, Juon, Gielen, Steinwachs, \& Duggan, 2008).

Statistically significant, and unique, associations were found for age as well. The youngest cohort was least likely to perceive a need for treatment when compared to all older age groups except women from ages 50 to 64 . Women in their twenties and early thirties were more likely to perceive a need for treatment than the youngest women. Women aged 35 to 49 were almost five times more likely to perceive a need for treatment than women aged 18 to 21 . This dramatic difference could be related to experiencing more effects of ongoing substance use at that age, especially if the women had started using when they were much younger. They may have reached a point in their lives where it appeared as though they may need help, but then experienced other barriers or obstacles which kept them out of treatment.

The association between marital status and perceived treatment need has never been explored for women who meet criteria for SUDs in the literature. According to the model, women who were widowed, divorced, or separated were $15 \%$ less likely to perceive a need for treatment than married women. Women who had never been married were $36 \%$ less likely to perceive a need for treatment than married women. Although these are small differences, they are still statistically significant and suggest that marriage is associated with a greater awareness among women that they may have a substance use problem. It may also be that these married women may have more responsibilities and 
obligations which are preventing them from being able to seek the treatment that they otherwise think they need.

Education has never been found to have a statistically significant association with perceived need for treatment in prior studies in this area, with education operationalized as either having graduated high school or not. However, the theoretical model by Andersen and Newman (1973/2005) suggested higher education is associated with higher treatment utilization although it is unclear which type of education they were referring to. They suggested that more education may be associated with a lifestyle and social environment more conducive to using health services. In the case of the women in this sample, it appeared as though having at least a high school diploma was associated with women being 1.71 times more likely to perceive a need for treatment than women who did not. However, despite having completed high school, these women reported needing treatment that they did not actually receive. It may be that these women experience other additional barriers which keep them out of treatment, such as gender-specific stigma. It may also be that by not distinguishing between women with graduate degrees versus women with undergraduate degrees or those who only graduated high school may not reveal the real differences between these groups of educated women. This is an area worth exploring further through research.

There were significant associations between what kinds of counties that women lived in and their perceived need for treatment, which had not been found in prior studies on this topic. Women from small metropolitan counties were $40 \%$ less likely than women from large metropolitan counties to perceive a need for treatment. Women from nonmetro/rural areas were $24 \%$ more likely to perceive a need for treatment when 
compared to women who lived in more concentrated areas. According to the theoretical model, living in a concentrated area can be enabling to women who already perceive a need for treatment because urban women could have more access to services if they want them. Meanwhile, it may be that rural women are more likely to perceive a need for treatment and not receive it due to issues with accessibility and transportation to treatment agencies.

Total family income had never before been found to be associated with the perceived treatment need of women; however it was in this study. Women in the sample who had family incomes below $\$ 20,000$ in the past year were $40 \%$ more likely to perceive a need for treatment than women with family incomes between $\$ 20,000$ and $\$ 49,999$. They were $20 \%$ more likely to perceive a need for treatment compared to women in families earning $\$ 50,000$ to $\$ 74,999$. Meanwhile, women from families earning over $\$ 75,000$ were $64 \%$ less likely to perceive a need for treatment when compared to women with the lowest family incomes. It is likely that this difference is due to the fact that women with access to more money may not bear the same negative consequences to their substance use as poorer women, thereby being less likely to perceive a problem. It is also likely that these women with more resources would be more likely to be able to afford the treatment if they wanted it too.

Women with health insurance were significantly but only slightly less likely to perceive a need for treatment when compared to women without health insurance. However, this is still a unique finding, as this had not been explored before. It may be that there are other factors which have stronger associations with perceived treatment need among women, yet it is still an enabling factor for women. 
In addition, women with anxiety in the past year were $88 \%$ more likely to perceive a need for treatment than women who did not experience it. Women who reported depression were $22 \%$ more likely to perceive a need for treatment than women who did not. These were both anticipated associations, based on the research literature. In addition, women who had received substance use treatment in the past were more likely by a factor of 2.73 to perceive a need for treatment than women who did not. This finding did not significantly distinguish women from the men in the sample, yet it is a finding that had not been explored much in the literature. It is worth exploring why women who have already received treatment and think they need it again are still not receiving it. These gender-specific issues could provide treatment providers with information on how to reach out to former clients who may be in need of services once again and to address potential barriers with resuming care.

In addition, substance use problem severity was significantly associated with perceived treatment need among women. The manner in which this was tested in this study is unique compared to other studies in this area. Women at the moderate level of substance use problem severity were twice as likely to perceive a need for treatment as at the mild level. Women at the highest level of problem severity were seven times more likely to perceive a need for treatment than women at the lowest level of problem severity. This suggests that some women with more severe substance-related problems are likely to believe that they are in need of treatment.

In summary, some predisposing and enabling factors were differently associated with perceived treatment need among women who met criteria for SUDs in this sample. Most findings are unique to this study due to its diverse and large sample, as well as the 
analyses which were conducted. This study's findings have important implications for understanding why there are differential rates of treatment enrollment among various subgroups of women and it also provides guidance for where intervention can help to increase the treatment utilization of women who actually want help for their substance use problems.

\section{Impact of predisposing and enabling factors on men}

No recent studies on the perceived treatment need of men have attempted to explore the impact of predisposing and enabling factors with such a large and diverse sample. This study found that, compared to non-Hispanic white men, Asian men and nonHispanic biracial/multiracial men were significantly less likely to perceive a need for treatment. As suggested earlier, it is likely that cultural attitudes and linguistic barriers may have contributed towards this difference. It appears as though non-Hispanic black men are $83 \%$ more likely to perceive a need for treatment when compared to nonHispanic white men. This is a noteworthy difference between genders in this racial/ethnic group worth exploring in future research. Native American and Alaska Native men are over two and half times more likely to perceive a need for treatment compared to nonHispanic white men. These specific minority men may encounter barriers or obstacles which have interfered with their ability to receive the treatment than they otherwise think they need. Efforts have been made to increase the number of treatment facilities on reservations, in rural areas, and in local languages to make treatment more accessible to Native Americans and Alaska Natives, however, there may be gender specific barriers which are still leading more men to stay out of treatment. It may be that cost and 
insurance access may limit their ability to pursue treatment. In addition, cultural elements may differentially impact these men who think they need treatment but still do not pursue it. These racial and ethnic differences are important to acknowledge in order to reduce the barriers to minority men.

There are less dramatic differences between the younger men in the sample. Of interest is that men in their early thirties were two and a half times more likely to perceive a need for treatment than the young men aged 18 to 21 . Men aged 35 to 49 were over three times more likely to believe they needed treatment when compared to the youngest men in the sample. Men aged 50 to 64 were almost three and a half times more likely to perceive a need for treatment compared to the men aged 18 to 21 , and men in the oldest age group were twice as likely to believe they needed treatment as the youngest men in the sample. These rates reveal that older men are generally more likely to perceive a need for treatment than the younger men in the sample. These men may be more likely to see their ongoing use as problematic as they get older and it interferes with other aspects of their lives, however, they may encounter barriers to access which prevent them from seeking care. These trends have not been documented in the perceived treatment need studies to date.

As noted earlier, the theoretical model suggested that marital status would be associated with perceived treatment need, but there was no prior research to confirm this. This study revealed that married men were $33 \%$ more likely than men who were widowed, divorced, or separated to perceive a need for treatment. Married men were almost $50 \%$ more likely to perceive a need for treatment compared to never married men. The presence of a spouse could help these men to become more aware that their 
substance use is becoming problematic, yet it may also be that these men face barriers which are still keeping them out of the treatment they think they need.

Education status had not been found to be significantly associated with perceived treatment need among men. This study suggests that men who graduated from high school were $32 \%$ less likely to perceive a need for treatment than men who had not graduated. This relationship could indicate that educated men were more likely to have accessed the care that they needed.

Whether someone resided in an urban, suburban or rural county was significantly associated with perceived treatment need, but it had a small association. Men whose family incomes were between $\$ 20,000$ and $\$ 74,999$ in the past year were roughly twice as likely to perceive a need for treatment as men from families in the lowest income bracket. It may be that middle class men experience more obstacles to actually pursuing the treatment they want, such as competing responsibilities at home and at work, or stigma. Men from the highest income group were as likely as men in the lowest income group to perceive a need for treatment.

Having insurance was associated with men being half as likely to perceive a need for treatment than men who did not. This association may exist because insured men were more likely to get the treatment that they wanted, rather than men who had no access. Having anxiety in the past year was associated with men being two and a half times more likely to perceive a need for treatment than if they did not. A similar association was found for depression as well. It appears as though having either of these co-occurring mental health problems increases the likelihood for perceiving a need for treatment among men, just as with women. Also, men who have received substance use 
treatment in the past are also more likely to perceive a need for treatment, as was found with women. Again, as in the case with women, this association should be explored to better understand why these individuals still do not access care they think they need despite having received it in the past.

As substance use problem severity increased, men were more likely to perceive a need for treatment. Men at the moderate level of problem severity were over three times more likely to perceive a need for treatment than men with mild problem severity. Men at high substance use problem severity were five and a half times more likely to perceive a need for treatment than men at the mild level of problem severity. This relationship is consistent with the relationship among perceived treatment need for women.

In summary, there are some notable differences in the association of various predisposing and enabling factors with perceived treatment need among men who meet criteria for SUDs. For example, there are some groups of minority men who perceive a need for treatment yet are still underrepresented in treatment settings. In addition, access to health insurance and education are differently associated with the perceived treatment need among men compared to women. Awareness of these differences can lead to increased strategies by social workers and public health workers to reduce disparities and increase access and utilization of treatment by those who want it most.

\section{Andersen and Newman's Model}

Andersen and Newman's model of individual determinants of healthcare utilization (1973/2005) was used as a guide to help select and include variables into the study. One strength of this model is that it acknowledges that health care utilization is not 
a simple behavior; instead, the model offers that a number of micro, mezzo, and macro level factors can influence and individual's ultimate decision to utilize healthcare services. It is not simply a psychological or medical model; it acknowledges that external factors such as health insurance, family values, community characteristics, and income play a role in decision-making.

However, other models could have been more appropriate for this study as well. Andersen and Newman's model is grounded in a medical perspective and was not specifically designed for social work. Although it does acknowledge a number of ecological factors, the model is not dynamic and interactive like social work's ecological perspective. Rather, the relationships between the variables are illustrated as unidirectional when these variables can have bidirectional influences upon one another. In addition, it was initially developed to explain healthcare utilization specific to medical and dental care, rather than mental health or substance use treatment. This could also lead to limited translation to this area of treatment. Another limitation of this model is that it does not necessarily seek to explain health disparities between genders or other minority groups and there are a number of other theories and models which have been used in this type of research before.

\section{Future studies}

Perceived treatment need is an important construct for future research, as it indicates that individuals have an insight into their substance use and believe that treatment could potentially be beneficial to them. These individuals are a small minority of substance users and are most likely to be receptive to referrals and outreach for 
treatment if approached properly. An improved understanding of the shared and unique beliefs of individuals in this situation can help to inform strategies to help them.

First of all, future studies on perceived treatment need should better operationalize 'treatment' when posing the question to participants. It may be that the participants understandings of what they believe treatment is or what treatment types are available are not accurate or do not represent the services which may actually be available to them. By asking about specific treatment modalities such as inpatient or outpatient, researchers can get a better idea of which types of treatment these individuals believe that they need. The most common reason stated by participants in the study for not pursuing treatment was because they were not ready to stop using drugs. It is possible that these individuals may be receptive to alternatives to abstinence-only treatment. It would be interesting to ask if individuals would be receptive to substance use treatment that was not necessarily abstinence-oriented, but would promote safer or managed use.

In addition, it would be interesting to further identify which factors (i.e. personal, familial, social, occupational) participants believed contributed to their perceiving a need for treatment. It would also be informative to know if participant responses would be different if they are asked whether someone else in their lives told them that they needed treatment. A study which uses a treatment-readiness scale such as The Stages of Change and Treatment Eagerness Scale (SOCRATES) could provide even more data about participant motivations around treatment and their perspectives about their substance use (Miller \& Tonnigan, 1996). A qualitative study could also capture the complexity of this issue as well. 
A longitudinal study to examine changes in perceived treatment need as well as changing patterns of substance use among participants would provide a great deal of information about when individuals perceive a need for treatment, whether they do eventually decide to quit, and whether they eventually seek treatment.

\section{Limitations}

There are a number of limitations associated with this study which should be considered when interpreting its results. The study utilized secondary data from an annually conducted cross-sectional survey on drug use and mental health of household residents in the United States. By selecting this data source for analysis, the study could only include research questions and develop hypotheses to fit within the variables which had already been gathered so the study was limited in this regard.

In addition, it was unclear when in the past year that the participant perceived the need for treatment. Perceiving a need for treatment six months ago versus perceiving a need for treatment within the past few days are two different things and would be very interesting to learn more about. Questions on gender identity, gender roles, and health beliefs were also not a part of this survey so that these variables could not be included to better inform the gender-related hypotheses. These limitations tie into the fact that this secondary data analysis was conducted using a survey containing only quantitative data, which lacked the richness of a mixed methods study. Collecting some qualitative responses for certain questions could have provided invaluable data about the perspectives of the participants. 
In addition, the study was limited because there was a lack of control over data coding and entry procedures, which resulted in the study providing primarily categorical values for analyses. This limited the types of analyses which could have been conducted. The fact that the survey was cross-sectional also limited questions and analyses which could be conducted, contributing to the choice of logistic regression for the primary analysis to answer the question. No inferences about causation could be made.

This study operationalized substance use problem severity by using the DSM-IV as its basis, however, this practice has limited generalizability beyond this year. The DSM-V has already been released and it includes three levels of problem severity under the general category of SUD (mild, moderate, and severe) rather than the current abuse and dependence diagnoses (Peer et al., 2013). In order to be diagnosed with a mild SUD, individuals will now have to meet two or three diagnostic criteria, a moderate SUD will require four to five, and a severe SUD would entail six or more. There are 11 total criteria, most of which have been taken from the existing abuse and dependence criteria. However, two noteworthy changes have taken place. First, the abuse criterion for recurrent legal problems within the past year has been removed and, second, the new diagnostic criterion for craving has been added in its place. All of these changes will impact the sum number of individuals who thereby will meet diagnostic criteria for a SUD and it will also come with its own premade classification system for problem severity. It would be beneficial if future studies could still consider that meeting diagnostic criteria for multiple substance use disorders still deserves recognition. 


\section{Implications for Social Work and Public Health}

Given the prevalence of substance use among Americans regardless of gender, age, race or ethnicity, this topic is relevant to social workers and other public health workers in a number of human service areas. Community-based substance users who perceive a need for treatment are an important population for social workers and other health workers to identify because it is likely that they would be receptive to referrals for care. In addition, understanding the social and cultural factors which may be preventing men from seeking the treatment they believe they need may be key to helping them to then access that care.

Research. Social work research is distinguished from other social science and clinical research for a number of reasons. It uses its ecological approach to acknowledge the impact of various micro (i.e. psychological, demographic), mezzo (i.e. familial, social, cultural), and macro (i.e. community, institutional) factors on human behavior. This study contributes to social work literature because it acknowledges the role of these important social and cultural markers which differentially impact the experiences of people. Based on the findings of this study, men at higher levels of problem severity are slightly more likely than similar women to have an unmet treatment need. In addition, most minority groups are more likely than non-Hispanic whites to perceive a need for treatment. Interestingly, they are often the ones who are also underrepresented in treatment settings. Research can help to uncover the differential barriers which deter these individuals from actually seeking the treatment and research can also uncover the best strategies for reducing barriers to access to various subgroups. 
In addition, social work's client-centered approach to treatment is predicated upon understanding how the client perceives their problems and 'starting where they're at.' Better understanding what exactly contributes to or is associated with perceiving a need for treatment among community substance users can help inform more research on factors which contribute to increased substance use treatment initiation, retention, and completion. These can allow for better and more effective strategies for referral and recruitment into treatment.

This study sought to identify whether women were more likely to interpret higher problem severity as indicative of needing substance use treatment. Although this hypothesis was not confirmed, this has opened up the possibility for further research on why, given increased problem severity and other factors, women are actually less likely than men to perceive a need for treatment. It would be beneficial for qualitative research to take on this issue to better understand the subjective experiences of these women. Women with severe substance use problems should be asked about their openness to pursue treatment with varying thresholds for treatment and whether non-abstinence oriented treatments would be appealing for them to at least begin addressing their problems. Also, their unique perceptions about the efficacy of treatment should be explored, as perhaps this may also have been a reason they did not pursue treatment. Additional quantitative research in this area can address the issue as well, but including additional variables and scales into model development to understand this phenomenon. In addition, qualitative studies with men at these higher levels of problem severity who are still not pursuing the treatment they need would also inform what can be done to get them into treatment. Further research in this area can better assist social workers and 
public health workers to understand the factors related to perceived treatment need, so that they can facilitate greater help-seeking among substance-using populations who are receptive to treatment.

Practice and teaching. Social work practitioners could potentially benefit from the findings of this study because problem severity and gender do impact perceptions of treatment need. It is important for social workers to be mindful of how they talk about substance use with clients of different genders. It may be that the approach to take when talking about substance use, substance-related problems, and substance use treatment should differ between female and male clients. Because it appears as though men are more likely to acknowledge their substance-related problems as potentially requiring treatment, social workers can use motivational enhancing techniques with these individuals or SBIRT to encourage them into treatment and to address whichever barriers they may be facing. If women are indeed less likely to perceive a need for treatment than men, social workers should be mindful about stirring up feelings of defensiveness with their approach in discussing the issue. Research on the best strategies to use to talk about substance use can better assist interviewers and clinicians on discussing substance use and its treatment. It is also important for social workers to explore more strategies and models for reaching specific racial and ethnic minority group members who perceive a need for treatment yet you are not receiving the care. Culturally sensitive approaches should continue to be developed and culturally competent social work students should continue to be trained to address the issue.

Individuals who perceive a need for substance use treatment and receive services from social workers in other settings should be identified. Social workers are already 
expected to conduct screenings for SUDs and to provide referrals to treatment and these clients (NASW, 2005). The use of SBIRT and other screening methods can help social workers in various settings to identify possible unmet treatment needs. In addition, these approaches are easy to teach to social work students for use in their future practice (Osborne \& Benner, 2012).

In addition, social workers should heed the information provided by the participants in the study which suggests that the most common reason for not pursuing treatment is because they are not ready to stop using. This could mean that these participants would be better suited for harm reduction-oriented treatments where they could work towards less riskier or reduced substance use instead of abstinence goals. Social workers and social work students should be trained in harm reduction and moderation strategies to be able to use them with clients who are resistant to abstinenceonly approaches.

Policy. Social workers and public health workers should together continue to endorse and support policies which reduce barriers to substance use treatment so that individuals who perceive a need for treatment can access it. The second most commonly reported reason for not receiving treatment among these participants was due to cost or lack of insurance. Social workers should advocate for public policies which equate behavioral health care with physical health care and expand health insurance coverage to low-income people who are not Medicaid eligible. In addition, social workers can advocate for more workplaces to allow for leave from employment if treatment is needed. Social workers should promote agency policies which also reduce barriers to clients, such as flexible treatment hours on evenings and weekends for individuals with family or work 
obligations, allowances for transportation, affordable payment plans for the uninsured, and flexible treatment plans which allow for harm reduction and moderation goals. By reducing the barriers to treatment, more individuals who perceive a need for it will choose to pursue it.

\section{Summary}

In summary, this study found that few individuals who meet criteria for SUDs do in fact perceive a need for treatment. However, there are some factors which are more associated with perceived treatment need than others. This study was distinguished from others on this subject in a number of ways. First, it consisted of a larger sample size than other studies in this area and the sample was very racially and ethnically diverse. A broad range of ages were also included, as well as participants from various parts of the United States. A number of theoretically informed variables were included in the analyses to understand the association between factors which may predispose some to perceive a need for treatment, as well as those factors which enable one to pursue the treatment that they want. Contrasts were made between those who perceived a need for treatment and those who did not. It was found that those who perceived a need for treatment generally had more severe problems, such as multiple SUDs. In addition, racial and other sociodemographic variables were associated with perceived treatment need. Gender differences were also explored this study, highlighting that various subgroups in each gender may be more or less likely to perceive a need for treatment. This is important information, as the number of women with SUDs is increasing and those who want treatment should be given access to that care. In addition, men with more severe 
substance use problems are more likely that women to perceive a need for treatment yet are still not pursuing it. Understanding this phenomenon is key to reducing unmet need among them. These variables can inform further attempts by social workers and public health workers to reduce healthcare disparities and to help individuals who want treatment to get it.

\section{Conclusion}

Not many people who meet criteria for SUDs want treatment for their problems. Those few who perceive a need for treatment are a select minority whose barriers to care should be reduced so that they can access the services they desire. Racial, ethnic, socioeconomic, and geographic factors contribute to the underrepresentation of certain people in treatment. Social workers and public health workers can use their skills and influence to address these issues and reduce barriers for greater access to care. 
Table 1

Descriptive Characteristics of Sample (Weighted and Unweighted)

Frequencies

$\begin{array}{ccc}\text { Variable } & \begin{array}{c}\text { Unweighted }(n=5,484) \\ \%\end{array} & \begin{array}{c}\text { Weighted }(n=20 \\ \%\end{array} \\ & & \\ & 60.8 & 59.6 \\ & 39.2 & 40.4\end{array}$

Substance Use Problem

Severity

Mild

43.8

44.2

Moderate

38.9

39.1

High

17.4

16.7

Race/Ethnicity

Non-Hispanic White

65.5

63.5

Non-Hispanic Black

32.3

10.2

Native American/Alaska

3.0

2.9

Native

Native Hawaiian

0.5

0.6

Islander/ Other

Pacific Islander

Asian

2.2

2.5

Non-Hispanic More

3.3

3.7

Than One Race

Hispanic

15.1

16.6

Age

18-21

36.8

37.7

22-25

32.8

32.5

26-29

7.1

7.3

30-34

6.9

7.6

$35-49$

12.3

11.1

50-64

3.5

3.4

65 or older

0.7

0.4

Marital Status

Married

15.7

29.0

Widowed, Divorced, or

8.8

17.9

Separated

Never Married

75.5

53.1 
County

Large metro

43.6

43.3

Small Metro

36.5

36.1

Nonmetro

19.9

20.6

Family Income

Less than $\$ 20,000$

33.1

35.1

$\$ 20,000-\$ 49,999$

32.3

31.1

$\$ 50,000-\$ 74,999$

14.2

15.3

$\$ 75,000$ or more

20.4

18.5

Has Health Insurance

72.9

74.3

Co-occurring Mental Health

Anxiety in Last Year

11.3

12.5

Depression in Last Year

12.8

12.7

Ever Received Substance

22.2

22.6

Use Treatment 
Table 2

Descriptive Characteristics of Sample, by Perceived Treatment Need and Gender

\begin{tabular}{|c|c|c|c|c|c|c|}
\hline \multirow[b]{3}{*}{ Characteristic } & \multicolumn{3}{|c|}{$\begin{array}{l}\text { Perceived Need for Treatment } \\
\qquad(n=986,498)\end{array}$} & \multicolumn{3}{|c|}{$\begin{array}{l}\text { No Perceived Need for Treatment } \\
\qquad(n=19,462,974)\end{array}$} \\
\hline & $\begin{array}{c}\text { Women } \\
(n=493,326)\end{array}$ & $\begin{array}{c}\text { Men } \\
(n=493,172)\end{array}$ & Total & $\begin{array}{c}\text { Women } \\
(n=7,772,808)\end{array}$ & $\begin{array}{c}\text { Men } \\
(n=11,690,166)\end{array}$ & Total \\
\hline & $\%$ & $\%$ & $\%$ & $\%$ & $\%$ & $\%$ \\
\hline $\begin{array}{r}\text { Substance Use } \\
\text { Problem } \\
\text { Severity }\end{array}$ & & & & & & \\
\hline Mild & 13.4 & 13.8 & 13.6 & 42.9 & 47.4 & 45.8 \\
\hline Moderate & 42.5 & 52.6 & 47.5 & 40.0 & 37.8 & 38.7 \\
\hline High & 44.1 & 33.6 & 38.9 & 17.1 & 14.5 & 15.6 \\
\hline Race/Ethnicity & & & & & & \\
\hline $\begin{array}{l}\text { Non-Hispanic } \\
\text { White }\end{array}$ & 59.5 & 63.2 & 61.4 & 63.8 & 63.5 & 63.6 \\
\hline $\begin{array}{l}\text { Non-Hispanic } \\
\text { Black }\end{array}$ & 6.0 & 14.2 & 10.1 & 10.3 & 10.1 & 10.2 \\
\hline $\begin{array}{l}\text { Native American/ } \\
\text { Alaska Native }\end{array}$ & 6.9 & 5.8 & 6.3 & 3.3 & 2.3 & 2.7 \\
\hline $\begin{array}{l}\text { Native Hawaiian } \\
\text { Islander/ Other } \\
\text { Pacific } \\
\text { Islander }\end{array}$ & 5.0 & 0.0 & 2.5 & 0.4 & 0.5 & 0.5 \\
\hline Asian & 0.0 & 0.4 & 0.2 & 2.0 & 3.1 & 2.6 \\
\hline $\begin{array}{c}\text { Non-Hispanic } \\
\text { More Than } \\
\text { One Race }\end{array}$ & 6.5 & 3.3 & 2.9 & 3.8 & 3.5 & 3.6 \\
\hline
\end{tabular}


Hispanic

Age

18-21

22-25

26-29

30-34

$35-49$

50-64

65 or older

Marital Status

Married

Widowed,

Divorced, or

Separated

Never Married

High school graduate

County

Large metro

Small Metro

Nonmetro

Family Income

Less than $\$ 20,000$

\$20,000-\$49,999

$\$ 50,000-\$ 74,999$

$\$ 75,000$ or more
16.0

13.2

14.6

16.3

16.9

16.6

27.6

23.7

22.0

6.0

17.7

23.1

7.2

0.4

25.7

27.1

6.5

11.2

25.1

4.2

0.2

41.7

32.5

6.4

6.0

10.8

2.4

0.3

36.9

20.8

26.4

19.7

42.3

53.9

81.9

66.0

73.7

42.6

33.0

24.4

45.7

29.5

24.8

45.0

37.8

17.2

34.2

37.0

19.2

9.7
23.8

19.7

10.6
8.8

38.4

38.4
29.7

13.7

18.2

36.1

33.0

38.3

\begin{tabular}{ll}
7.4 & 7.3 \\
\hline
\end{tabular}

$10.1 \quad 10.4$

$3.9 \quad 3.3$

$0.5 \quad 0.5$

30.1

28.6

16.5

17.8

53.4

53.6

80.8

81.2

42.0

35.6

43.2

22.4

36.5

20.4 
Has Health Insurance

77.3

44.1

43.5

Year

Depression in Last Year

Ever Received

Substance Use

Treatment

51.4
55.7

28.9

25.4

63.4

57.4

7.4

6.6

11.1

35.0

20.3

5.9

11.8

0.8 
Table 3

Characteristics of Perceived Treatment Need Group by Gender $(n=986,498)$

\begin{tabular}{|c|c|c|c|c|c|c|c|c|}
\hline \multirow[b]{2}{*}{ Characteristic } & \multicolumn{2}{|c|}{$\begin{array}{c}\text { Women } \\
(n=493,326) \\
\end{array}$} & \multicolumn{2}{|c|}{$\begin{array}{c}\text { Men } \\
(n=493,172) \\
\end{array}$} & \multirow[t]{2}{*}{$\chi^{2}$} & \multirow[t]{2}{*}{$d f$} & \multirow[t]{2}{*}{$\mathrm{p}$} & \multirow[t]{2}{*}{$V$} \\
\hline & $\mathrm{n}$ & $\%$ & $\mathrm{n}$ & $\%$ & & & & \\
\hline Substance Use & & & & & $12,212.77$ & 2 & $<0.001$ & 0.111 \\
\hline Problem Severity & & & & & & & & \\
\hline Mild & 66,110 & 13.4 & 68,065 & 13.8 & & & & \\
\hline Moderate & 209,769 & 42.5 & 259,292 & 52.6 & & & & \\
\hline High & 217,447 & 44.1 & 165,815 & 33.6 & & & & \\
\hline Race/Ethnicity & & & & & $50,275.74$ & 6 & $<0.001$ & 0.226 \\
\hline $\begin{array}{c}\text { Non-Hispanic } \\
\text { White }\end{array}$ & 293,767 & 59.5 & 311,918 & 63.2 & & & & \\
\hline $\begin{array}{l}\text { Non-Hispanic } \\
\text { Black }\end{array}$ & 29,751 & 6.0 & 69,903 & 14.2 & & & & \\
\hline $\begin{array}{c}\text { Native American/ } \\
\text { Alaska Native }\end{array}$ & 34,126 & 6.9 & 28,433 & 5.8 & & & & \\
\hline $\begin{array}{l}\text { Native Hawaiian } \\
\text { Islander/ Other } \\
\text { Pacific Islander }\end{array}$ & 24,567 & 5.0 & 0 & 0.0 & & & & \\
\hline Asian & 0 & 0.0 & 1,833 & 0.4 & & & & \\
\hline $\begin{array}{c}\text { Non-Hispanic } \\
\text { More Than } \\
\text { One Race }\end{array}$ & 32,060 & 6.5 & 16,149 & 3.3 & & & & \\
\hline Hispanic & 79.055 & 16.0 & 64,935 & 13.2 & & & & \\
\hline Age & & & & & $72,179.52$ & 6 & $<0.001$ & 0.270 \\
\hline $18-21$ & 136,323 & 27.6 & 116,860 & 23.7 & & & & \\
\hline $22-25$ & 158,871 & 32.2 & 108,512 & 22.0 & & & & \\
\hline $26-29$ & 34,901 & 7.1 & 29,526 & 6.0 & & & & \\
\hline $30-34$ & 23,366 & 4.7 & 87,278 & 17.7 & & & & \\
\hline $35-49$ & 133,565 & 27.1 & 113,676 & 23.1 & & & & \\
\hline $50-64$ & 6,299 & 1.3 & 35,352 & 7.2 & & & & \\
\hline 65 or older & 0 & 0.0 & 1,967 & 0.4 & & & & \\
\hline Marital Status & & & & & & & & \\
\hline Married & 151,508 & 30.7 & 212,429 & 43.1 & $34,215.30$ & 2 & $<0.001$ & 0.186 \\
\hline $\begin{array}{l}\text { Widowed, } \\
\text { Divorced, or } \\
\text { Separated }\end{array}$ & 137,453 & 27.9 & 67,556 & 13.7 & & & & \\
\hline Never Married & 204,365 & 41.4 & 213,187 & 43.2 & & & & \\
\hline
\end{tabular}




\begin{tabular}{|c|c|c|c|c|c|c|c|c|}
\hline $\begin{array}{l}\text { High school } \\
\text { graduate }\end{array}$ & 402,047 & 81.5 & 325,274 & 66.0 & $30,754.25$ & 1 & $<0.001$ & 0.177 \\
\hline $\begin{array}{l}\text { County } \\
\text { Large metro } \\
\text { Small Metro } \\
\text { Nonmetro }\end{array}$ & $\begin{array}{l}241,293 \\
127,705 \\
124,327\end{array}$ & $\begin{array}{l}48.9 \\
25.9 \\
25.2\end{array}$ & $\begin{array}{l}209,933 \\
162,944 \\
120,295\end{array}$ & $\begin{array}{l}42.6 \\
33.0 \\
24.4\end{array}$ & $6,518.40$ & 2 & $<0.001$ & 0.081 \\
\hline $\begin{array}{l}\text { Family Income } \\
\text { Less than } \\
\$ 20,000 \\
\$ 20,000-\$ 49,999 \\
\$ 50,000-\$ 74,999 \\
\$ 75,000 \text { or more }\end{array}$ & $\begin{array}{c}219,727 \\
138,218 \\
92,080 \\
43,300\end{array}$ & $\begin{array}{c}44.5 \\
\\
28.0 \\
18.7 \\
8.8\end{array}$ & $\begin{array}{c}117,331 \\
226,673 \\
97,126 \\
52,042\end{array}$ & $\begin{array}{l}23.8 \\
46.0 \\
19.7 \\
10.6\end{array}$ & $53,486.15$ & 3 & $<0.001$ & 0.233 \\
\hline $\begin{array}{l}\text { Has Health } \\
\text { Insurance }\end{array}$ & 381,567 & 77.3 & 274,814 & 55.7 & $51,785.08$ & 1 & $<0.001$ & 0.229 \\
\hline \multicolumn{9}{|l|}{$\begin{array}{l}\text { Co-occurring Mental } \\
\text { Health }\end{array}$} \\
\hline $\begin{array}{l}\text { Anxiety in Last } \\
\text { Year }\end{array}$ & 217,687 & 44.1 & 142,579 & 28.9 & $28,408.39$ & 1 & $<0.001$ & 0.171 \\
\hline $\begin{array}{l}\text { Depression in } \\
\text { Last Year }\end{array}$ & 214,469 & 43.5 & 125,201 & 25.4 & $40,097.95$ & 1 & $<0.001$ & 0.203 \\
\hline $\begin{array}{l}\text { Ever Received } \\
\text { Substance Use } \\
\text { Treatment }\end{array}$ & 253,375 & 51.4 & 312,881 & 63.4 & $14,722.95$ & 1 & $<0.001$ & 0.122 \\
\hline
\end{tabular}


Table 4

Characteristics of No Perceived Need for Treatment Group by Gender $(n=19,462,974)$

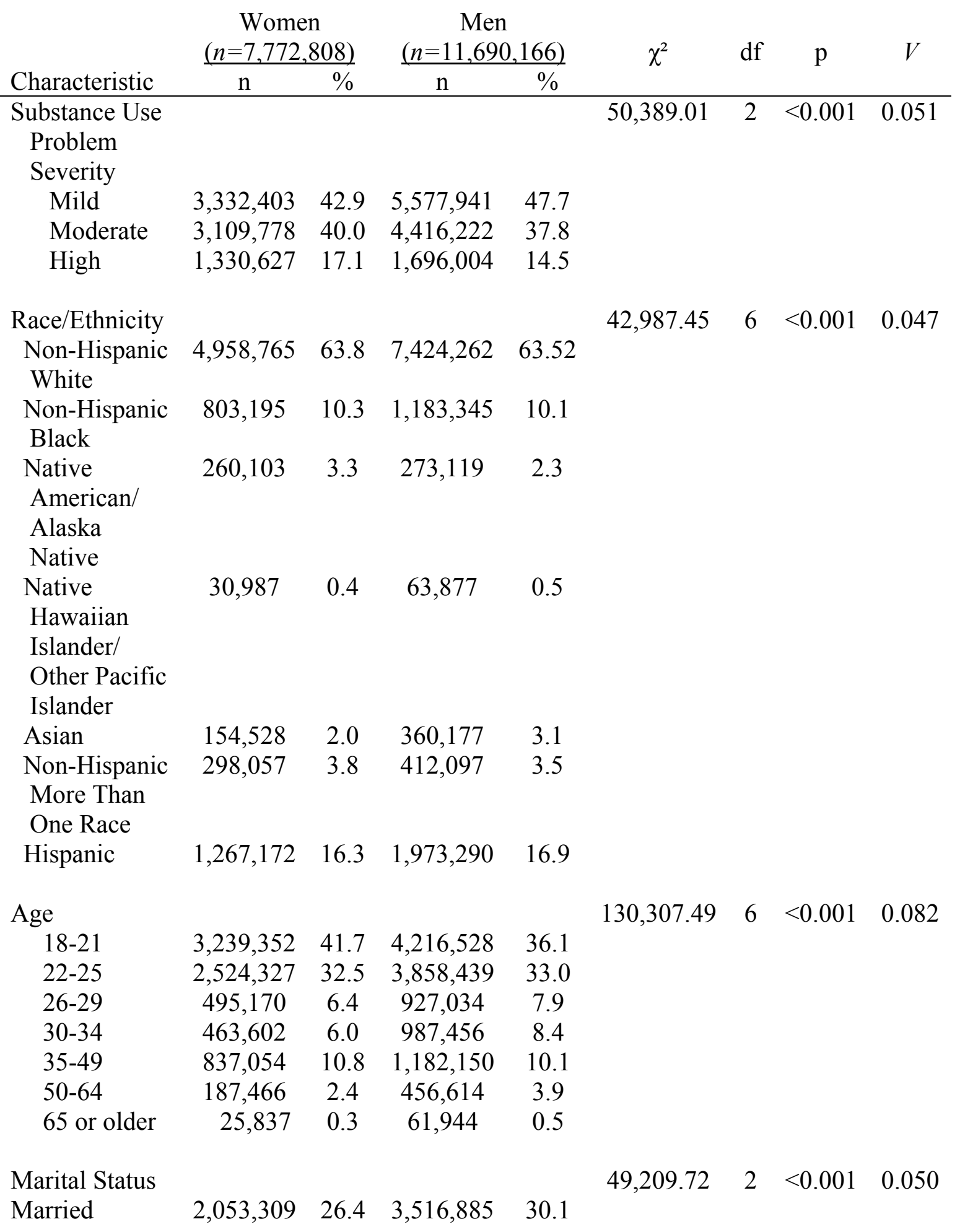




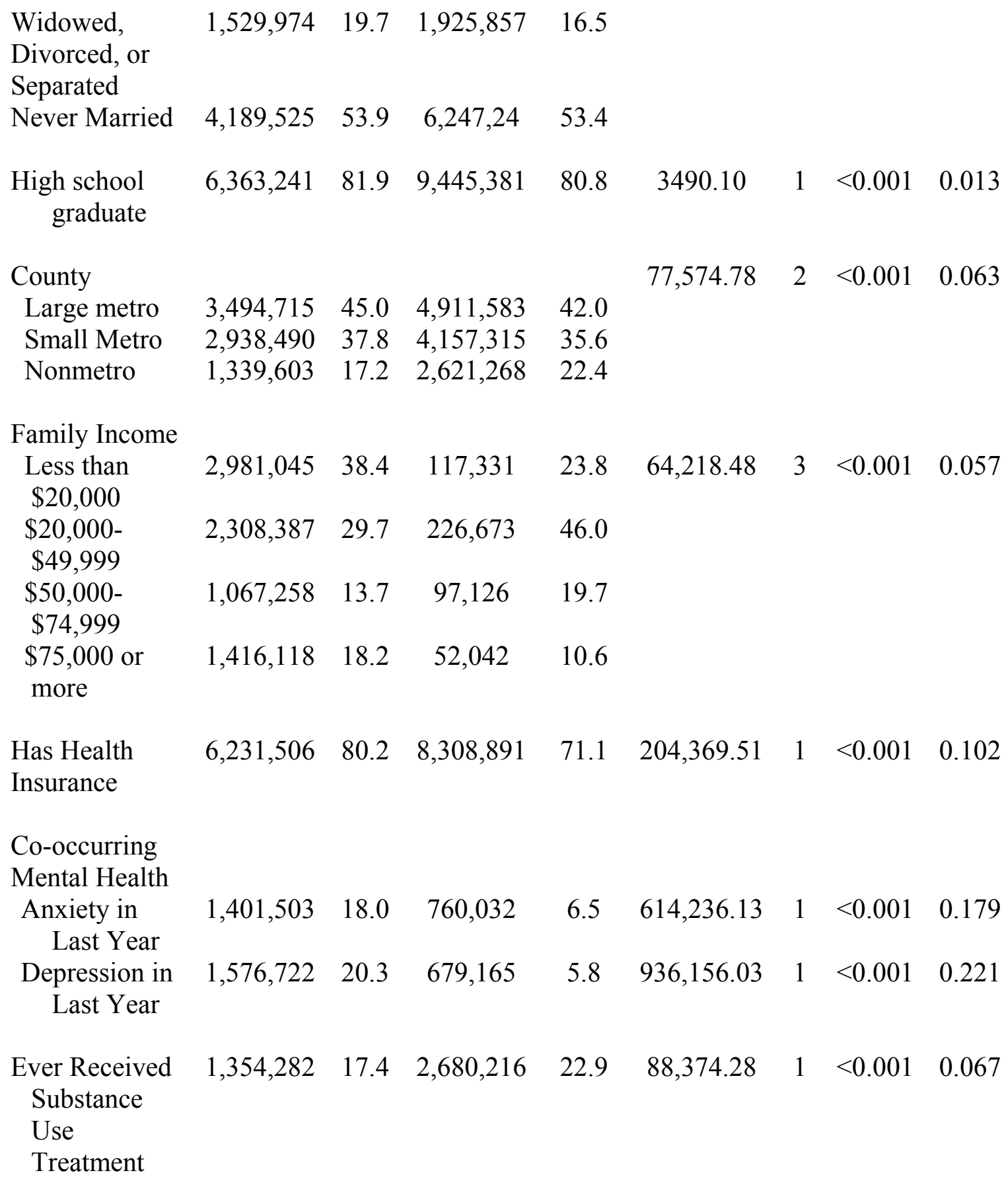


Table 5

Most Commonly Reported Reasons for not Receiving Substance Use Treatment among Those who Perceived a Need for Treatment In Descending Order ${ }^{1}$

\begin{tabular}{lcc} 
Reason & $n$ & $\%$ \\
\hline Not ready to stop using & 389,767 & 39.5 \\
Could not afford treatment/Had no insurance & 329,977 & 33.4 \\
Neighbors would have a negative opinion of & 129,079 & 13.1 \\
me if they found out & & 11.0 \\
I could handle the problem without & 108,633 & 10.6 \\
treatment & 104,553 & 10.1 \\
No transportation & 99,236 & 9.6 \\
I didn't feel the need for treatment at the & & 9.1 \\
time & 94,956 & 7.4 \\
It would have a negative effect on my job & 90,075 & 7.2 \\
Didn't know where to go & 72,521 & 6.3 \\
Didn't want others to know & 71,253 & 5.7 \\
I didn't have time & 62,357 & 4.4 \\
Insurance didn't cover full treatment cost & 56,690 & 3.4 \\
No openings in the program & 43,312 & \\
No program had the type of treatment I & 34,002 & \\
wanted & & \\
I didn't think it would help & & \\
\end{tabular}

\footnotetext{
${ }^{1}$ Note: Including duplicates. Percentage reported is out of number of participants who perceived a need for treatment $(n=986,498)$. They comprise $4.8 \%$ of total sample.
} 
Table 6

Most Commonly Reported Reasons for not Receiving Substance Use Treatment among Women who Perceived a Need for Treatment In Descending Order ${ }^{2}$

$\begin{array}{lcc}\text { Reason } & n & \% \\ \text { Not ready to stop using } & 234,507 & 47.5 \\ \text { Could not afford treatment/Had no insurance } & 172,781 & 35.0 \\ \text { I could handle the problem without } & 77,556 & 15.7 \\ \text { treatment } & & 14.5 \\ \text { It would have a negative effect on my job } & 71,308 & 12.8 \\ \text { No transportation } & 63,300 & 12.8 \\ \text { Didn't want others to know } & 63,296 & 11.6 \\ \text { Neighbors would have a negative opinion of } & 57,057 & 10.3 \\ \text { me if they found out } & & \\ \text { I didn't feel the need for treatment at the } & 50,791 & 9.7 \\ \text { time } & & 8.6 \\ \text { Didn't know where to go } & 47,629 & 5.8 \\ \text { I didn't have time } & 42,642 & 5.2 \\ \text { I didn't think it would help } & 28,603 & 25,899 \\ \text { No program had the type of treatment I } & & 5.1 \\ \text { wanted } & 25,114 & 3.2 \\ \text { Insurance didn't cover full treatment cost } & 15,903 & \end{array}$

\footnotetext{
${ }^{2}$ Note: Including duplicates. Percentage reported is out of number of female participants who perceived a need for treatment $(n=493,326)$.
} 
Table 7

Most Commonly Reported Reasons for not Receiving Substance Use Treatment among Men who Perceived a Need for Treatment In Descending Order ${ }^{3}$

\begin{tabular}{lcc} 
Reason & $n$ & $\%$ \\
\hline Could not afford treatment/Had no insurance & 157,196 & 31.9 \\
Not ready to stop using & 155,260 & 31.5 \\
Neighbors would have a negative opinion of & 72,022 & 14.6 \\
me if they found out & 48,445 & 9.8 \\
I didn't feel the need for treatment at the & & 8.6 \\
time & 42,446 & 8.4 \\
Didn't know where to go & 41,253 & 8.3 \\
No transportation & 40,787 & 6.3 \\
No openings in the program & 31,077 & \\
I could handle the problem without & & 5.8 \\
treatment & 28,612 & 4.8 \\
I didn't have time & 23,648 & 3.5 \\
It would have a negative effect on my job & 17,413 & \\
No program had the type of treatment I & & \\
wanted & 9,225 & 1.9 \\
Didn't want others to know & 5,399 & 1.1
\end{tabular}

${ }^{3}$ Note: Including duplicates. Percentage reported is out of number of male participants who perceived a need for treatment $(n=493,172)$. 
Table 8

Bivariate Correlations Between Variables ${ }^{4}$

\begin{tabular}{|c|c|c|c|c|c|c|c|c|c|c|c|c|c|}
\hline Variable & 1 & 2 & 3 & 4 & 5 & 6 & 7 & 8 & 9 & 10 & 11 & 12 & 13 \\
\hline 1. Gender & 1.00 & & & & & & & & & & & & \\
\hline 2. Race/ & 0.049 & 1.00 & & & & & & & & & & & \\
\hline Ethnicity & & & & & & & & & & & & & \\
\hline 3. Age & -0.114 & 0.060 & 1.00 & & & & & & & & & & \\
\hline $\begin{array}{l}\text { 4. Marital } \\
\text { Status }\end{array}$ & 0.057 & 0.095 & 0.062 & 1.00 & & & & & & & & & \\
\hline 5. Education & 0.021 & 0.261 & -0.017 & 0.036 & 1.00 & & & & & & & & \\
\hline $\begin{array}{l}\text { 6. County } \\
\text { Type }\end{array}$ & -0.040 & 0.178 & -0.040 & 0.030 & -0.022 & 1.00 & & & & & & & \\
\hline 7. Income & -0.039 & 0.125 & 0.130 & 0.058 & 0.080 & -0.150 & 1.00 & & & & & & \\
\hline 8. Insurance & 0.107 & 0.140 & 0.272 & 0.041 & 0.167 & -0.011 & 0.079 & 1.00 & & & & & \\
\hline 9. Anxiety & 0.182 & 0.122 & -0.028 & 0.027 & -0.002 & -0.017 & 0.010 & 0.054 & 1.00 & & & & \\
\hline 10.Depression & 0.222 & 0.093 & -0.072 & 0.021 & 0.006 & -0.009 & 0.002 & 0.063 & 0.456 & 1.00 & & & \\
\hline $\begin{array}{l}\text { 11. Treatment } \\
\text { Ever }\end{array}$ & -0.061 & 0.116 & -0.028 & 0.010 & -0.081 & 0.038 & -0.029 & -0.071 & 0.149 & 0.162 & 1.00 & & \\
\hline $\begin{array}{l}\text { 12. Perceived } \\
\text { Treatment } \\
\text { Need }\end{array}$ & 0.044 & 0.081 & -0.012 & 0.049 & -0.041 & -0.000 & -0.005 & -0.041 & 0.167 & 0.148 & 0.188 & 1.00 & \\
\hline $\begin{array}{l}\text { 13. Substance } \\
\text { Use Problem } \\
\text { Severity }\end{array}$ & 0.059 & 0.071 & -0.060 & 0.036 & -0.052 & -0.020 & -0.040 & -0.015 & 0.097 & 0.074 & 0.131 & 0.059 & 1.00 \\
\hline
\end{tabular}

${ }^{4}$ Conducted using weighted data, $n=20,077,235$ and all $p$ values for all correlations were less than 0.001 
Table 9

Main Effect of Substance Use Problem Severity upon Perceived Treatment Need $(n=20,077,235)$

\begin{tabular}{|c|c|c|c|c|c|c|c|c|}
\hline \multirow[b]{2}{*}{ Variables } & \multirow[b]{2}{*}{$B$} & \multirow[b]{2}{*}{$\mathrm{SE}$} & \multirow[b]{2}{*}{ Wald } & \multirow[b]{2}{*}{$d f$} & \multirow[b]{2}{*}{ Sig. } & \multirow[b]{2}{*}{ OR } & \multicolumn{2}{|c|}{$95 \% \mathrm{CI}$} \\
\hline & & & & & & & LL & UL \\
\hline $\begin{array}{l}\text { Substance use problem severity (reference: } \\
\text { mild) }\end{array}$ & & & 434535.619 & 2 & .001 & & & \\
\hline Moderate Severity & 1.420 & .003 & 205253.102 & 1 & .001 & 4.139 & 4.114 & 4.164 \\
\hline High Severity & 2.129 & .003 & 431619.505 & 1 & .001 & 8.409 & 8.356 & 8.463 \\
\hline Constant & -4.196 & .003 & 2327105.115 & 1 & .001 & .015 & & \\
\hline
\end{tabular}


Table 10

Main Effect of Gender upon Perceived Treatment Need $(n=20,077,235)$

\begin{tabular}{|c|c|c|c|c|c|c|c|c|}
\hline \multirow[b]{2}{*}{ Variables } & \multirow[b]{2}{*}{$B$} & \multirow[b]{2}{*}{ SE } & \multirow[b]{2}{*}{ Wald } & \multirow[b]{2}{*}{$d f$} & \multirow[b]{2}{*}{ Sig. } & \multirow[b]{2}{*}{ OR } & \multicolumn{2}{|c|}{$95 \% \mathrm{CI}$} \\
\hline & & & & & & & LL & UL \\
\hline Gender (reference: men) & & & & & & & & \\
\hline Women & .408 & .002 & 39076.252 & 1 & .001 & 1.504 & 1.498 & 1.511 \\
\hline Constant & -3.166 & .001 & 4742170.886 & 1 & .001 & .042 & & \\
\hline
\end{tabular}


Table 11

Interaction Effect of Substance Use Problem Severity and Gender upon Perceived Treatment Need $(n=20,077,235)$

\begin{tabular}{|c|c|c|c|c|c|c|c|c|}
\hline \multirow[b]{2}{*}{ Variables } & \multirow[b]{2}{*}{$B$} & \multirow[b]{2}{*}{ SE } & \multirow[b]{2}{*}{ Wald } & \multirow[b]{2}{*}{$d f$} & \multirow[b]{2}{*}{ Sig. } & \multirow[b]{2}{*}{ OR } & \multicolumn{2}{|c|}{$95 \% \mathrm{CI}$} \\
\hline & & & & & & & LL & UL \\
\hline Substance Use Problem Severity (reference: & & & 203475.047 & 2 & .001 & & & \\
\hline Mild Severity) & & & & & & & & \\
\hline Moderate Severity & 1.571 & .004 & 130214.964 & 1 & .001 & 4.812 & 4.771 & 4.853 \\
\hline High Severity & 2.081 & .005 & 201491.028 & 1 & .001 & 8.012 & 7.940 & 8.085 \\
\hline Gender (reference: men) & 0.486 & .006 & 7795.536 & 1 & .001 & 1.626 & 1.608 & 1.643 \\
\hline Substance Use Problem Severity x Gender & & & 7608.332 & 2 & .001 & & & \\
\hline Gender x Moderate Severity & -0.347 & .006 & 3054.296 & 1 & .001 & 0.707 & .698 & .715 \\
\hline Gender x High Severity & 0.028 & .007 & 18.180 & 1 & .001 & 1.028 & 1.015 & 1.041 \\
\hline Constant & -4.406 & .004 & 1305473.224 & 1 & .001 & 0.012 & & \\
\hline
\end{tabular}


Table 12

Interaction of Effect of Substance Use Problem Severity and Gender upon Perceived Treatment Need in Addition to Predisposing and Enabling Factors $(n=19,981,581)$

\begin{tabular}{|c|c|c|c|c|c|c|c|c|}
\hline \multirow[b]{2}{*}{ Variables } & \multirow[b]{2}{*}{$B$} & \multirow[b]{2}{*}{ SE } & \multirow[b]{2}{*}{ Wald } & \multirow[b]{2}{*}{$d f$} & \multirow[b]{2}{*}{ Sig. } & \multirow[b]{2}{*}{ OR } & \multicolumn{2}{|c|}{$95 \% \mathrm{CI}$} \\
\hline & & & & & & & $\mathrm{LL}$ & UL \\
\hline $\begin{array}{l}\text { Race/Ethnicity (reference: Non-Hispanic } \\
\text { White) }\end{array}$ & & & 84683.71 & 6 & .001 & & & \\
\hline Non-Hispanic Black & 0.04 & .00 & 110.04 & 1 & .001 & 1.04 & 1.04 & 1.05 \\
\hline Native American/Alaska Native & 0.62 & .01 & 15005.02 & 1 & .001 & 1.85 & 1.83 & 1.87 \\
\hline $\begin{array}{l}\text { Native Hawaiian Islander/ Other Pacific } \\
\text { Islander }\end{array}$ & 2.30 & .01 & 60508.94 & 1 & .001 & 9.95 & 9.77 & 10.13 \\
\hline Asian & -1.84 & .02 & 6067.75 & 1 & .001 & 0.16 & 0.15 & 0.17 \\
\hline Non-Hispanic More Than One Race & 0.40 & .01 & 5352.48 & 1 & .001 & 1.50 & 1.48 & 1.51 \\
\hline Hispanic & 0.24 & .00 & 5056.21 & 1 & .001 & 1.27 & 1.27 & 1.28 \\
\hline Age (reference: $18-21$ years) & & & 184095.57 & 6 & .001 & & & \\
\hline $22-25$ & 0.22 & .00 & 4962.64 & 1 & .001 & 1.25 & 1.24 & 1.26 \\
\hline $26-29$ & 0.24 & .01 & 2520.71 & 1 & .001 & 1.27 & 1.26 & 1.29 \\
\hline $30-34$ & 0.98 & .00 & 55453.51 & 1 & .001 & 2.67 & 2.65 & 2.69 \\
\hline $35-49$ & 1.26 & .00 & 140856.30 & 1 & .001 & 3.53 & 3.51 & 3.56 \\
\hline $50-64$ & 0.96 & .01 & 23982.58 & 1 & .001 & 2.60 & 2.56 & 2.63 \\
\hline 65 or older & 0.41 & .02 & 305.424 & 1 & .001 & 1.50 & 1.44 & 1.57 \\
\hline Marital Status (reference: married) & & & 44670.18 & 2 & .001 & & & \\
\hline Widowed, Divorced, or Separated & -0.26 & .00 & 6271.32 & 1 & .001 & 0.77 & 0.77 & 0.78 \\
\hline Never Married & -0.54 & .00 & 44388.80 & 1 & .001 & 0.58 & 0.58 & 0.59 \\
\hline High school graduate & -0.08 & .00 & 877.90 & 1 & .001 & 0.92 & 0.92 & 0.93 \\
\hline County (reference: large metro) & & & 18443.85 & 2 & .001 & & & \\
\hline & & 153 & & & & & & \\
\hline
\end{tabular}


Small Metro

Nonmetro

Family Income (reference: less than $\$ 20,000$ )

$$
\$ 20,000-\$ 49,999
$$

$\$ 50,000-\$ 74,999$

$\$ 75,000$ or more

Has health insurance

Anxiety in Last Year

Depression in Last Year

Ever Received Substance Use Treatment

Substance use problem severity (reference: mild)

Moderate Severity

High Severity

Gender

Gender x Substance Use Problem Severity Gender x Moderate Severity Gender x High Severity

Constant

$\begin{array}{cccccccc}-0.34 & .00 & 15553.49 & 1 & .001 & 0.72 & 0.71 & 0.72 \\ 0.01 & .00 & 5.38 & 1 & .02 & 1.01 & 1.00 & 1.01 \\ & & & & & & & \\ & & 36106.58 & 3 & .001 & & & \\ 0.14 & .00 & 2726.53 & 1 & .001 & 1.15 & 1.15 & 1.16 \\ 0.30 & .00 & 7732.88 & 1 & .001 & 1.36 & 1.35 & 1.36 \\ -0.49 & .00 & 14245.82 & 1 & .001 & 0.61 & 0.61 & 0.62 \\ & & & & & & & \\ -0.45 & .00 & 30950.55 & 1 & .001 & 0.64 & 0.63 & 0.65 \\ 0.77 & .00 & 73877.478 & 1 & .001 & 2.15 & 2.14 & 2.17 \\ 0.49 & .00 & 27383.23 & 1 & .001 & 1.63 & 1.62 & 1.64 \\ 1.04 & .00 & 192770.68 & 1 & .001 & 2.83 & 2.82 & 2.84 \\ & & 148667.06 & 2 & & & & \\ 1.26 & .01 & 78149.11 & 1 & .001 & 3.53 & 3.49 & 3.56 \\ 1.90 & .01 & 148542.84 & 1 & .001 & 6.69 & 6.63 & 6.75 \\ & & & & & & & \\ 0.52 & .01 & 8086.47 & 1 & .001 & 1.67 & 1.66 & 1.69 \\ & & 10726.42 & 2 & .001 & & & \\ -0.58 & .01 & 7606.59 & 1 & .001 & 0.56 & .56 & .57 \\ -0.16 & .01 & 557.79 & 1 & .001 & 0.85 & 0.84 & .86 \\ -4.59 & .01 & 579318.41 & 1 & .001 & .01 & & \end{array}$


Table 13

Interaction of Effect of Substance Use Problem Severity upon Perceived Treatment Need in Addition to Predisposing and Enabling Factors for Women in Sample $(n=8,172,619)$

\begin{tabular}{|c|c|c|c|c|c|c|c|c|}
\hline \multirow[b]{2}{*}{ Variables } & \multirow[b]{2}{*}{$B$} & \multirow[b]{2}{*}{ SE } & \multirow[b]{2}{*}{ Wald } & \multirow[b]{2}{*}{$d f$} & \multirow[b]{2}{*}{ Sig. } & \multirow[b]{2}{*}{ OR } & \multicolumn{2}{|c|}{$95 \% \mathrm{CI}$} \\
\hline & & & & & & & $\mathrm{LL}$ & UL \\
\hline $\begin{array}{l}\text { Race/Ethnicity (reference: Non-Hispanic } \\
\text { White) }\end{array}$ & & & $105,026.05$ & 6 & .001 & & & \\
\hline Non-Hispanic Black & -0.81 & .01 & $12,775.46$ & 1 & .001 & 0.44 & 0.44 & 0.45 \\
\hline Native American/Alaska Native & 0.19 & .01 & 674.31 & 1 & .001 & 1.21 & 1.19 & 1.22 \\
\hline $\begin{array}{l}\text { Native Hawaiian Islander/ Other Pacific } \\
\text { Islander }\end{array}$ & 2.90 & .01 & $64,496.80$ & 1 & .001 & 18.12 & 17.72 & 18.53 \\
\hline Asian & -71.60 & 99.43 & 0.03 & 1 & .860 & 0.00 & 0.00 & $9.94 \mathrm{e} 76$ \\
\hline Non-Hispanic More Than One Race & 1.14 & .01 & $23,526.58$ & 1 & .001 & 3.11 & 3.07 & 3.16 \\
\hline Hispanic & 0.44 & .01 & $8,288.77$ & 1 & .001 & 1.56 & 1.54 & 1.57 \\
\hline Age (reference: $18-21$ years) & & & $124,951.35$ & 6 & .001 & & & \\
\hline $22-25$ & 0.45 & .00 & $10,568.14$ & 1 & .001 & 1.57 & 1.56 & 1.59 \\
\hline $26-29$ & 1.04 & .01 & $22,819.26$ & 1 & .001 & 2.82 & 2.78 & 2.86 \\
\hline $30-34$ & 1.00 & .01 & $15,932.07$ & 1 & .001 & 2.70 & 2.66 & 2.75 \\
\hline $35-49$ & 1.61 & .01 & $112,435.52$ & 1 & .001 & 4.99 & 4.94 & 5.03 \\
\hline $50-64$ & -0.43 & .02 & 361.61 & 1 & .001 & 0.65 & 0.62 & 0.68 \\
\hline 65 or older & -16.84 & 241.75 & 0.01 & 1 & .944 & 0.00 & 0.00 & $2.90 \mathrm{e} 19$ \\
\hline Marital Status (reference: married) & & & $13,489.76$ & 2 & .001 & & & \\
\hline Widowed, Divorced, or Separated & -0.18 & .01 & 1489.76 & 1 & .001 & 0.84 & 0.83 & 0.85 \\
\hline Never Married & -0.45 & .00 & $12,924.83$ & 1 & .001 & 0.64 & 0.63 & 0.64 \\
\hline High school graduate & 0.54 & .01 & $14,128.62$ & 1 & .001 & 1.71 & 1.69 & 1.72 \\
\hline County (reference: large metro) & & & 26,232 & 2 & .001 & & & \\
\hline & & 155 & & & & & & \\
\hline
\end{tabular}


Small Metro

Nonmetro

Family Income (reference: less than $\$ 20,000$ ) $\$ 20,000-\$ 49,999$ $\$ 50,000-\$ 74,999$

$\$ 75,000$ or more

Has health insurance

Anxiety in Last Year

Depression in Last Year

Ever Received Substance Use Treatment

Substance use problem severity (reference: mild)

Moderate Severity

High Severity

Constant

$\begin{array}{cccccccc}-0.52 & .00 & 16,510.45 & 1 & .001 & 0.60 & 0.59 & 0.60 \\ 0.21 & .00 & 2,272.25 & 1 & .001 & 1.24 & 1.23 & 1.25 \\ & & & & & & & \\ -0.52 & .00 & 15,823.06 & 1 & .001 & 0.60 & 0.59 & 0.60 \\ -0.22 & .01 & 1902.79 & 1 & .001 & 0.80 & 0.80 & 0.81 \\ -0.99 & .01 & 27,759.30 & 1 & .001 & 0.37 & 0.37 & 0.38 \\ & & & & & & & \\ -0.16 & .00 & 1,518.63 & 1 & .001 & 0.85 & 0.84 & 0.86 \\ 0.63 & .00 & 25,338.93 & 1 & .001 & 1.88 & 1.87 & 1.90 \\ 0.20 & .00 & 2,333.87 & 1 & .001 & 1.22 & 1.21 & 1.23 \\ 1.00 & .00 & 75,569.58 & 1 & .001 & 2.73 & 2.71 & 2.75 \\ & & 184,744.24 & 2 & & & & \\ 0.74 & .01 & 22,587.86 & 1 & .001 & 2.09 & 2.07 & 2.11 \\ 1.96 & .01 & 154,185.92 & 1 & .001 & 7.13 & 7.06 & 7.20 \\ -4.59 & .01 & 290,907.16 & 1 & .001 & .01 & & \end{array}$


Table 14

Interaction of Effect of Substance Use Problem Severity upon Perceived Treatment Need in Addition to Predisposing and Enabling Factors for Men in Sample $(n=11,904,617)$

\begin{tabular}{|c|c|c|c|c|c|c|c|c|}
\hline \multirow[b]{2}{*}{ Variables } & \multirow[b]{2}{*}{$B$} & \multirow[b]{2}{*}{ SE } & \multirow[b]{2}{*}{ Wald } & \multirow[b]{2}{*}{$d f$} & \multirow[b]{2}{*}{ Sig. } & \multirow[b]{2}{*}{ OR } & \multicolumn{2}{|c|}{$95 \% \mathrm{CI}$} \\
\hline & & & & & & & $\mathrm{LL}$ & $\mathrm{UL}$ \\
\hline $\begin{array}{l}\text { Race/Ethnicity (reference: Non-Hispanic } \\
\text { White) }\end{array}$ & & & $36,167.17$ & 6 & .001 & & & \\
\hline Non-Hispanic Black & 0.61 & .01 & $15,054.68$ & 1 & .001 & 1.83 & 1.81 & 1.85 \\
\hline Native American/Alaska Native & 0.95 & .01 & $16,378.03$ & 1 & .001 & 2.58 & 2.54 & 2.62 \\
\hline $\begin{array}{l}\text { Native Hawaiian Islander/ Other Pacific } \\
\text { Islander }\end{array}$ & -16.38 & 166.98 & 0.01 & 1 & .922 & 0.00 & 0.00 & $1.04 \mathrm{e} 135$ \\
\hline Asian & -1.35 & 0.02 & 3208.38 & 1 & .001 & 0.26 & 0.25 & 0.27 \\
\hline Non-Hispanic More Than One Race & -0.60 & .01 & $3,431.40$ & 1 & .001 & 0.55 & 0.54 & 0.56 \\
\hline Hispanic & 0.08 & .01 & 233.52 & 1 & .001 & 1.08 & 1.07 & 1.09 \\
\hline Age (reference: $18-21$ years) & & & $96,964.53$ & 6 & .001 & & & \\
\hline $22-25$ & 0.16 & .01 & $1,060.26$ & 1 & .001 & 1.17 & 1.16 & 1.18 \\
\hline $26-29$ & -0.15 & .01 & 424.73 & 1 & .001 & 0.86 & 0.85 & 0.88 \\
\hline $30-34$ & 0.94 & .01 & $30,503.30$ & 1 & .001 & 2.55 & 2.52 & 2.58 \\
\hline $35-49$ & 1.15 & .01 & $50,494.40$ & 1 & .001 & 3.15 & 3.12 & 3.18 \\
\hline $50-64$ & 1.25 & .01 & $31,334.58$ & 1 & .001 & 3.49 & 3.44 & 3.54 \\
\hline 65 or older & 0.78 & .02 & 1054.27 & 1 & .001 & 2.19 & 2.09 & 2.29 \\
\hline Marital Status (reference: married) & & & $33,343.53$ & 2 & .001 & & & \\
\hline Widowed, Divorced, or Separated & -0.41 & .01 & $6,277.16$ & 1 & .001 & 0.67 & 0.66 & 0.67 \\
\hline Never Married & -0.65 & .00 & $33,274.38$ & 1 & .001 & 0.52 & 0.52 & 0.53 \\
\hline High school graduate & -0.39 & .00 & $10,713.40$ & 1 & .001 & 0.68 & 0.67 & 0.68 \\
\hline County (reference: large metro) & & & $4,398.63$ & 2 & .001 & & & \\
\hline & & 157 & & & & & & \\
\hline
\end{tabular}




\begin{tabular}{|c|c|c|c|c|c|c|c|c|}
\hline Small Metro & -0.23 & .00 & $3,744.66$ & 1 & .001 & 0.79 & 0.79 & 0.80 \\
\hline Nonmetro & -0.21 & .00 & $2,320.04$ & 1 & .001 & 0.81 & 0.80 & 0.82 \\
\hline Family Income (reference: less than $\$ 20,000$ ) & & & $42,922.24$ & 3 & .001 & & & \\
\hline$\$ 20,000-\$ 49,999$ & 0.67 & .00 & $26,869.72$ & 1 & .001 & 1.96 & 1.94 & 1.97 \\
\hline$\$ 50,000-\$ 74,999$ & 0.81 & .01 & $23,824.18$ & 1 & .001 & 2.24 & 2.22 & 2.27 \\
\hline$\$ 75,000$ or more & 0.34 & .01 & 32.49 & 1 & .001 & 1.04 & 1.02 & 1.04 \\
\hline Has health insurance & -0.69 & .00 & $38,751.22$ & 1 & .001 & 0.50 & 0.50 & 0.51 \\
\hline Anxiety in Last Year & 0.94 & .00 & $49,780.08$ & 1 & .001 & 2.56 & 2.54 & 2.58 \\
\hline Depression in Last Year & 0.92 & .00 & $43,321.36$ & 1 & .001 & 2.50 & 2.48 & 2.52 \\
\hline Ever Received Substance Use Treatment & 1.14 & .00 & 116,653 & 1 & .001 & 3.14 & 3.12 & 3.16 \\
\hline $\begin{array}{l}\text { Substance use problem severity (reference: } \\
\text { mild) }\end{array}$ & & & $110,801.53$ & 2 & & & & \\
\hline Moderate Severity & 1.17 & .01 & $64,635.68$ & 1 & .001 & 3.23 & 3.21 & 3.26 \\
\hline High Severity & 1.71 & .01 & $109,146.28$ & 1 & .001 & 5.54 & 5.48 & 5.59 \\
\hline Constant & -4.55 & .01 & $355,667.76$ & 1 & .001 & .01 & & \\
\hline
\end{tabular}


Figure 1. Individual Determinants of Health Service Utilization

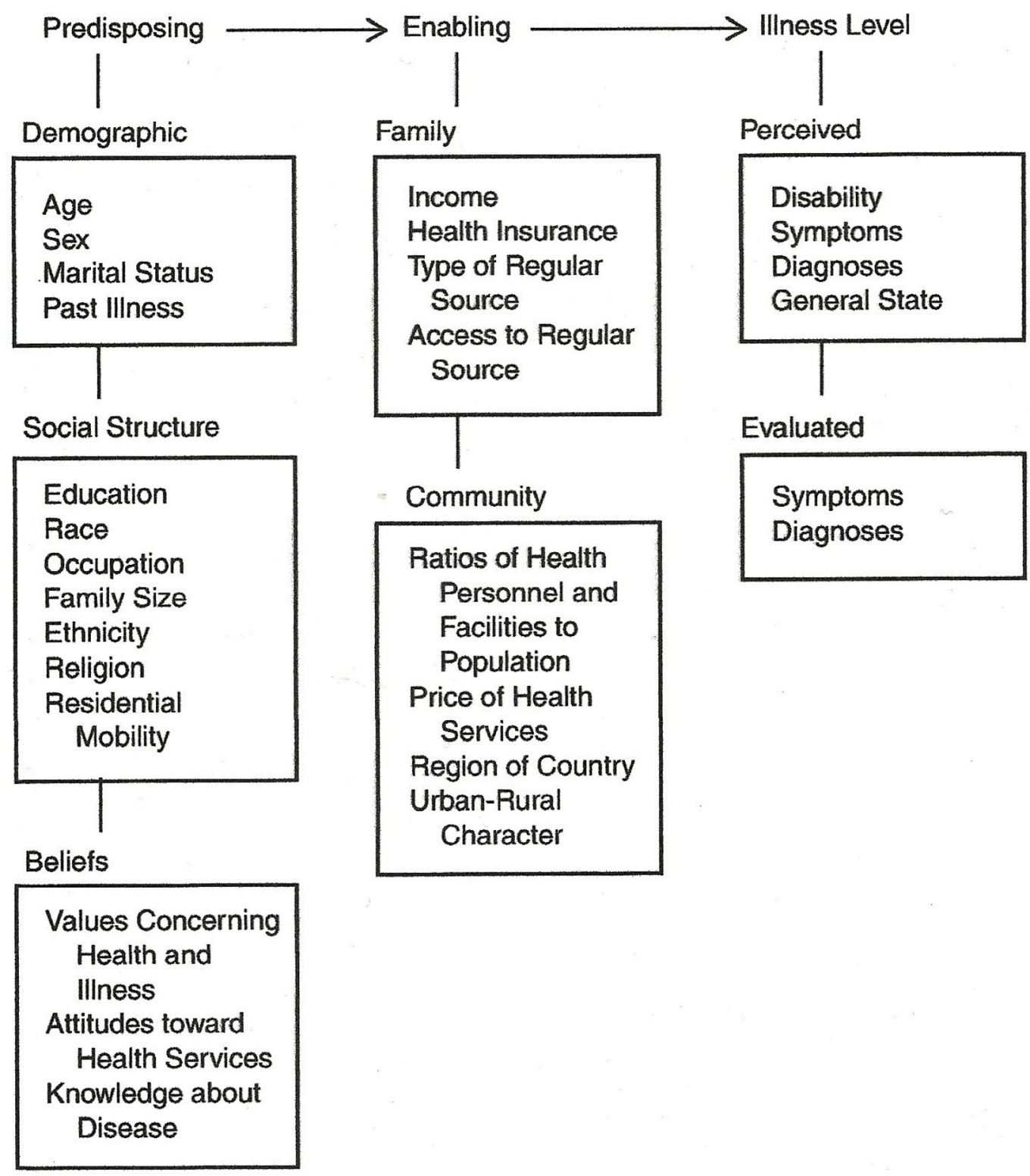

Reprinted with permission from "Societal and Individual Determinants of Medical Care Utilization in the United States," by R. Andersen and J. F. Newman, 2005, The Milbank Quarterly, 83, p. 14. Copyright 2005 by Milbank Memorial Fund 


\section{REFERENCES}

Abbott, M. L. \& McKinney, J. (2013). Understanding and applying research design. Hoboken, NJ: John Wiley \& Sons.

Addis, M. E. \& Mahalik, J. R. (2003). Men, masculinity, and the contexts of help seeking. American Psychologist, 58(1), 5-14. doi: 10.1037/0003-066X.58.1.5

American Psychiatric Association. (2000). Diagnostic and statistical manual of mental disorders: DSM-IV-TR. Washington, DC: Author.

Andersen, R. \& Newman, J. F. (2005). Societal and individual determinants of medical care utilization in the United States. The Milbank Quarterly, 83(4), 1-28. (Reprinted from The Milbank Memorial Fund Quarterly: Health and Society, 51(1) 1973, 95-124)

Anthony, J. C. \& Helzer, J. E. (1991). Syndromes of drug abuse and dependence. In L. N. Robins \& D. A. Regier (Eds.). Psychiatric disorders in America: The Epidemiologic Catchment Area study (pp. 116-154). New York: Free Press.

Aquaro, G. D., Gabutti, A., Meini, M., Prontera, C., Pasanisi, E., Passino, C., Emdin, M. \& Lombardi, M. (2011). Silent myocardial damage in cocaine addicts. Heart. Advance online publication. doi: 10.1136/hrt.2011.226977

Babor, T. F., Caulkins, J., Edwards, G., Fischer, B., Foxcroft, D., Humphreys, K., Obot, I., Rehm, J., Reuter, P., Room, R., Rossow, I. \& Strang, J. (2010). Drug policy and the public good. New York: Oxford University Press.

Babor, T. F. \& Higgins-Biddle, J. C. (2000). Alcohol screening and brief intervention: Dissemination strategies for medical practice and public health. Addiction, 95(5), 677-696.

Babor, T. F., McRee, B. G., Kassebaum, P. A., Grimaldi, P. L., Ahmed, K. \& Bray, J. (2007). Screening, brief intervention, and referral to treatment (SBIRT): Toward a public health approach to the management of substance abuse. Substance Abuse, 28(3), 7-30. doi:10.1300/J465v28n03_03

Ball, S. A., Carroll, K. M., Canning-Ball, M. \& Rounsaville, B. J. (2006). Reasons for dropout from drug abuse treatment: Symptoms, personality, and motivation. Addictive Behaviors, 31, 320-330. doi:10.1016/j.addbeh.2005.05.013

Becker, J. B. \& Hu, M. (2008). Sex differences in drug abuse. Frontiers in neuroendocrinology, 29, 36-47. doi: 10.1016/j.yfrne.2007.07.003 
Begun, A. L., Rose, S. J., LeBel, T. P. \& Teske-Young, B. A. (2009). Implementing substance abuse screening and brief motivational intervention with women in jail. Journal of Social Work Practice in the Addictions, 9(1), 113-131. doi: 10.1080/15332560802533372

Bertakis, K. D., Azari, R., Helms, J., Callahan, E. J. \& Robbins, J. A. (2000). Gender differences in the utilization of health care services. The Journal of Family Practice, 49(2), 147-152.

Bhadra, P. K., Millery, M., Scimeca, M. \& Polissar, N. L. (2002). Predicting long-term treatment utilization among addicts entering detoxification: The contribution of help-seeking models. Journal of Drug Issues, 32(1), 209-230.

Brace, N., Kemp, R. \& Snelgar, R. (2009). SPSS for psychologists $\left(4^{\text {th }}\right.$ ed.). New York: Routledge.

Brady, K. T. \& Randall, C. L. (1999). Gender differences in substance use disorders. Addictive Disorders, 22(2), 241-252.

Broyles, L. M. \& Gordon, A. J. (2010). SBIRT implementation: Moving beyond the interdisciplinary rhetoric. Substance Abuse, 31(4), 221-223. doi: 10.1080/08897077.2010.514238

Bryan, A., Kagee, A. \& Broaddus, M. R. (2006). Condom use among South African adolescents: Developing and testing theoretical models of intentions and behavior. AIDS and Behavior, 10(4), 387-197.

Bureau of Justice Statistics (2010). Drugs and crime. Retrieved from: http://bjs.ojp.usdoj.gov/index.cfm?ty $=$ tp $\&$ tid $=35$

Burns, R. B. \& Burns, R. A. (2009). Business research methods and statistics using SPSS. London, England: Sage Publications.

Carey, K. B., Scott-Sheldon, L. A. J., Elliott, J. C., Bolles, J. R. \& Carey, M. P. (2009). Computer-delivered interventions to reduce college student drinking: A metaanalysis. Addiction, 104, 1807-1819. doi:10.1111/j.1360-0443.2009.02691.x

Centers for Disease Control and Prevention (2002). Drug-associated HIV transmission continues in the United States. Retrieved from http://www.cdc.gov/hiv/resources/factsheets/PDF/idu.pdf

Centers for Disease Control and Prevention (2011). Impaired Driving: Get the facts. Retrieved from http://www.cdc.gov/MotorVehicleSafety/Impaired_Driving/impaireddrv_factsheet.html. 
Chen, P., Cribb, D., Dai, L., Gordek, H., Laufenberg, J., Sathe, N., \& Westlake, M. (2012). Person-level sampling weight calibration. In 2010 National Survey on Drug Use and Health: Methodological resource book. Section 12, prepared for the Substance Abuse and Mental Health Services Administration under Contract No. HHSS283200800004C, Phase I, Deliverable No. 39, RTI/0211838.107.004). Research Triangle Park, NC: RTI International.

Cohen, E., Feinn, R., Arias, A. \& Kranzler, H. R. (2007). Alcohol treatment utilization: Findings from the National Epidemiological Survey on Alcohol and Related Conditions. Drug and Alcohol Dependence, 86, 214-221. doi:10.1016/j.drugalcdep.2006.06.008

Collins, R. L. \& McNair, L. D. (2002). Minority women and alcohol use. Alcohol Research and Health, 26(4), 251-256.

Corrigan, P. W., Kuwabara, S. A., \& O'Shaughnessy, J. (2009). The public stigma of mental illness and drug addiction. Journal of Social Work, 9(2), 139-147.

Coulson, C., Ng, F., Geertsema, M., Dodd, S. \& Berk, M. (2009). Client-reported reasons for non-engagement in drug and alcohol treatment. Drug and Alcohol Review, 28, 372-378. doi: 10.1111/j.1465-3362.2009.00054.x

Courtenay, W. H. (2000a). Engendering health: A social constructionist examination of men's health beliefs and behaviors. Psychology of Men and Masculinity, 1(1), 415 .

Courtenay, W. H. (2000b). Constructions of masculinity and their influence on men's well-being: A theory of gender and health. Social Science \& Medicine, 50, 13851401.

Courtenay, W. H. (2003). Key determinants of the health and well-being of men and boys. International Journal of Men's Health, 2(1), 1-30.

Courtenay, W. H., McCreary, D. R. \& Merighi, J. R. (2002). Gender and ethnic differences in health beliefs and behaviors. Journal of Health Psychology, 7(3), 219-231. doi: 10.1177/1359105302007003216

Davenport-Hines, R. (2001). The pursuit of oblivion: A global history of narcotics 15002000. London: Weidenfeld \& Nicolson.

Dawson, D. A. \& Grant, B. F. (2010). Should symptom frequency be factored into scalar measures of alcohol use disorder severity? Addiction, 105, 1568-1579. doi: 10.1111/j.1360-0443.2010.02994.x 
Dawson, D. A., Grant, B. F., Stinson, F. S., Chou, P. S., Huang, B. \& Ruan, W. J. (2005). Recovery from DSM-IV alcohol dependence: United States, 2001-2002. Addiction, 100(3), 281-292. doi: 10.1111/j.1360-0443.2004.00964.x

Dawson, D. A., Saha, T. D. \& Grant, B. F. (2010). A multidimensional assessment of the validity and utility of alcohol use disorder severity as determined by item response theory models. Drug and Alcohol Dependence, 107, 31-38. doi: 10.1016/j.drugalcdep.2009.08.019

Deci, E. L., \& Ryan, R. M. (1985). Intrinsic motivation and self-determination in human behavior. New York: Plenum.

DeGrandpre, R. (2006). The cult of pharmacology. Durham: Duke University Press.

De Leeuw, E., Hox, J., \& Kef, S. (2003). Computer-assisted self-interviewing tailored for special populations and topics. Field Methods, 15, 223-251. doi: $10.1177 / 1525822 X 03254714$

Dennis, B. S., Buchholtz, A. K. \& Butts, M. M. (2009). The nature of giving: A theory of planned behavior examination of corporate philanthropy. Business \& Society, 48(3), 360-384.

Druss, B. G., Wang, P. S., Sampson, N. A., Olfson, M., Pincus, H. A., Wells, K . B. \& Kessler, R. C. (2007). Understanding mental health treatment in persons without mental diagnoses: Results from the National Comorbidity Survey Replication. Archives of General Psychiatry, 64(10), 1196-1203.

Edlund, M. J., Unutzer, J. \& Curran, G. M. (2006). Perceived need for alcohol, drug, and mental health treatment. Social Psychiatry and Psychiatric Epidemiology, 41, 480-487.

Elhai, J. D. \& Ford, J. D. (2007). Correlates of mental health service use intensity in the National Comorbidity Survey and National Comorbidity Survey Replication. Psychiatric Services, 58(8), 1108-1115.

Escobar, J. I. \& Vega, W. A. (2007). Cultural issues and psychiatric diagnosis: Providing a general background for considering substance use diagnoses. Addiction, 101 (Supplement 1), 40-47.

Falck, R. S., Wang, J., Carlson, R. G., Krishnan, L. L., Leukefeld, C. \& Booth, B. M. (2007). Perceived need for substance abuse treatment among illicit stimulant drug users in rural areas of Ohio, Arkansas, and Kentucky. Drug and Alcohol Dependence, 91(2-3), 107-114. doi:10.1016/j.drugalcdep.2007.05.015 
Farabee, D., Shen, H. \& Sanchez, S. (2002). Perceived coercion and treatment need among mentally ill parolees. Criminal Justice and Behavior, 29(1), 76-86. doi: $10.1177 / 0093854802029001005$

Faul, F., Erdfelder, E., Lang, A. G. \& Buchner, A. (2007). G*Power 3: A flexible statistical power analysis program for the social, behavioral, and biomedical sciences. Behavior Research Methods, 39(2), 175-191.

Faul, F., Erdfelder, E., Buchner, A. \& Lang, A. G. (2009). Statistical power analyses using G*Power 3.1: Tests for correlation and regression analyses. Behavioral Research Methods, 41(4), 1149-1160. doi: 10.3758/BRM.41.4.1149

Federal Bureau of Investigation Uniform Crime Reporting Program (2010). Crime in the United States. Retrieved from: http://www2.fbi.gov/ucr/cius2009/arrests/index.html

Frances, A. (1998). Problems in defining clinical significance in epidemiological studies. Archives of General Psychiatry, 55, 119.

Frazier, P. A., Tix, A. P. \& Barron, K. E. (2004). Testing moderator and mediator effects in counseling psychology research. Journal of Counseling Psychology, 51(1), 115-134. doi: 10.1037/0022-0167.51.1.115

Freeman, E. M. (2001). Substance abuse intervention, prevention, rehabilitation, and systems change strategies: Helping individuals, families, and groups to empower themselves. New York: Columbia University Press.

Gerdner, A. \& Holmberg, A. (2000). Factors affecting motivation to treatment in severely dependent alcoholics. Journal of Studies on Alcohol, 61, 548-560.

Gfroerer, J. C. \& Epstein, J. F. (1999). Marijuana initiates and their impact on future drug abuse treatment need. Drug and Alcohol Dependence, 54, 229-237.

Gfroerer, J., Penne, M., Pemberton, M. \& Folsom, R. (2003). Substance abuse treatment need among older adults in 2020: The impact of the aging baby-boom cohort. Drug and Alcohol Dependence, 69, 127-135.

Gillespie, N. A., Neale, M. C., Prescott, C. A., Aggen, S. H. \& Kendler, K. S. (2007). Factor and item-response analysis of DSM-IV criteria for abuse of and dependence on cannabis, cocaine, hallucinogens, sedatives, stimulants and opioids. Addiction, 102, 920-930. doi: 10.1111/j.1360-0443.2007.01804.x

Green, C. A. (2006). Gender and use of substance abuse treatment services. Alcohol Research and Health, 29(1), 55-62. 
Green, C. A., Polen, M. R., Dickinson, D. M., Lynch, F. L \& Bennett, M. D. (2002). Gender differences in predictors of initiation, retention, and completion in an HMO-based substance abuse treatment program. Journal of Substance Abuse Treatment, 23, 285-295.

Greenfield, S. F., Brooks, A. J., Gordon, S. M., Green, C. A., Kropp, F., McHugh, R. K., ... Miele, G. M. (2007). Substance abuse treatment entry, retention, and outcome in women: A review of the literature. Drug and Alcohol Dependence, 86, 1-21. doi:10.1016/j.drugalcdep.2006.05.012

Gregoire, T. K. \& Burke, A. C. (2004). The relationship of legal coercion to readiness to change among adults with alcohol and other drug problems. Journal of Substance Abuse Treatment, 26, 35-41. doi: 10.1016/S0740-5472(03)00155-7

Grella, C. E., Cochran, S. D., Greenwell, L. \& Mays, V. M. (2011). Effects of sexual orientation and gender on perceived need for treatment by persons with and without mental disorders. Psychiatric Services, 62(4), 404-410.

Grella, C. E., Karno, M. P., Warda, U. S., Moore, A. A. \& Niv, N. (2009). Perceptions of need and help received for substance dependence in a national probability survey. Psychiatric Services, 60, 1068-1074.

Groshkova, T. (2010). Motivation in substance misuse treatment. Addiction Research \& Theory, 18(5), 494-510. doi: 10.3109/16066350903362875

Grucza, R. A., Norberg, K. E. \& Bierut, L. J. (2009). Binge drinking among youth and young adults in the United States : 1979-2009. Journal of American Academy of Child and Adolescent Psychiatry, 48(7), 692-702. doi: 10.1097/CHI.0b013e3181a2b32f

Havassy, B. E., Alvidrez, J. \& Mericle, A. A. (2009). Disparities in use of mental health and substance abuse services by persons with co-occurring disorders. Psychiatric Services, 60(2), 217-223.

Helzer, J. E., Burnam, A. \& McEvoy, L. T. (1991). Alcohol abuse and dependence. In L. N. Robins \& D. A. Regier (Eds.). Psychiatric disorders in America: The Epidemiologic Catchment Area study (pp. 81-115). New York: Free Press.

Hester, R. K., Delaney, H. D. \& Campbell, W. (2011). Moderatedrinking.com and moderation management: Outcomes of a randomized clinical trial with nondependent problem drinkers. Journal of Consulting and Clinical Psychology, 79, 215-224. doi: $10.1037 / \mathrm{a} 0022487$

Hintze, J. (2011). PASS 11. Kaysville, UT: NCSS, LLC. 
Humes, K. R., Jones, N. A. \& Ramirez, R. R. (2011). Overview of Race and Hispanic Origin: 2010 Census Briefs. Retrieved from: http://www.census.gov/prod/cen2010/briefs/c2010br-02.pdf

Hyejeong, K. \& Karpova, E. (2010). Consumer attitudes toward fashion counterfeits: Application of the theory of planned behavior. Clothing and Textiles Research Journal, 28(2), 79-94.

The InSight Project Research Group (2009). SBIRT outcomes in Houston: Final Report on InSight, a hospital district-based program for patients at risk for alcohol or drug use problems. Alcoholism: Clinical and Experimental Research, 33(8), 1374-1381. doi: 10.1111/j.1530-0277.2009.00967.x

Jaccard, J. (2001). Interaction effects in logistic regression. (Sage University Papers Series on Quantitative Applications in the Social Sciences, series no. 07-135). Thousand Oaks, CA: Sage.

Jackson, S. L. (2012). Research methods and statistics: A critical thinking approach (4 $4^{\text {th }}$ ed.). Belmont, CA: Wadsworth.

Jones, J. D., Mogali, S. \& Comer, S. D. (2012). Polydrug abuse: A review of opioid and benzodiazepine combination use. Drug and Alcohol Dependence, 125, 8-18. http://dx.doi.org/10.1016/j.drugalcdep.2012.07.004

Kessler, R. C., Crum, R. M., Warner, L. A., Nelson, C. B., Schulenberg, J. \& Anthony, J. C. (1997). Lifetime co-occurrence of DSM-III-R alcohol abuse and dependence with other psychiatric disorders in the National Comorbidity Survey. Archives of General Psychiatry, 54, 313-321.

Kessler, R. C., McGonagle, K. A., Zhao, S., Nelson, C. B., Hughes, M., Eshleman, S., Wittchen, H. U. \& Kendler, K. S. (1994). Lifetime and 12-month prevalence of DSM-III-R psychiatric disorders in the United States: Results from the National Comorbidity Survey. Archives of General Psychiatry, 51, 8-19.

Kessler, R. C., Chiu, W. T., Demler, O. \& Walters, E. E. (2005). Prevalence, severity and comorbidity of 12-month DSM-IV disorders in the National Comorbidity Survey Replication. Archives of General Psychiatry, 62, 617-627.

King, G. \& Zeng, L. (2001a). Explaining rare events in international relations. International Organization, 55(3), 693-715.

King, G. \& Zeng, L. (2001b). Logistic regression in rare events data. Political Analysis, $9(2), 137-162$.

Klein, A. (2008). Drugs and the world. London: Reaktion Books Ltd. 
Koob, G. F. (2006). The neurobiology of addiction: A neuroadaptational view relevant for diagnosis. Addiction, 101 (Supplement 1), 23-30.

Longshore, D., Hsieh, S. C., Anglin, M. D. \& Annon, T. A. (1992). Ethnic patterns in drug abuse treatment utilization. Journal of Mental Health Administration, 19(3), 268-277.

Madras, B. K., Compton, W. M., Avula, D., Stegbauer, T., Stein, J. B. \& Clark, H. W. (2009). Screening, brief interventions, referral to treatment (SBIRT) for illicit drug and alcohol use at multiple healthcare sites: Comparison at intake and 6 months later. Drug and Alcohol Dependence, 99, 280-295. doi: 10.1016/j.drugalcdep.2008.08.003

Mansfield, A. K., Addis, M. E. \& Mahalik, J. R. (2003). "Why won't he go to the doctor?": The psychology of men's help seeking. International Journal of Men's Health, 2(2), 93-100.

Mansfield, A. K., Addis, M. E. \& Courtenay, W. H.(2005). Measurement of men's help seeking: Development and evaluation of the Barriers to Help Seeking Scale. Psychology of Men \& Masculinity, 6(2), 95-108. doi: 10.1037/1524-9220.6.2.95

Marlatt, G. A. \& Witkiewitz, K. (2010). Update on harm-reduction policy and intervention research. Annual Review of Clinical Psychology, 6, 591-606. doi: 10.1146/annurev.clinpsy.121208.131438

Miller, W. R. (1983). Motivational interviewing with problem drinkers. Behavioral Psychotherapy, 11, 147-172.

Miller, W. R., Forcehimes, A. A. \& Zweben, A. (2011). Treating addiction: A guide for professionals. New York: Guilford Press.

Miller, W. R. \& Rollnick, S. (1991). Motivational interviewing: Preparing people to change addictive behavior. New York: Guilford Press.

Miller, W. R. \& Tonigan, J. S. (1996). Assessing drinkers' motivation for change: The Stages of Change Readiness and Treatment Eagerness Scale (SOCRATES). Psychology of Addictive Behaviors, 10(2), 81-89.

Mojtabai, R., Olfson, M. \& Mechanic, D. (2002). Perceived need and help-seeking in adults with mood, anxiety, or substance use disorders. Archives of General Psychiatry, 59, 77-84.

Muehlenkamp, J. J., Walsh, B. W. \& McDade, M. (2010). Preventing non-suicidal selfinjury in adolescents: The signs of self-injury program. Journal of Youth and Adolescence, 39(3), 306-314. 
Musto, D. F. (1973). The American disease. New Haven, CT: Yale University Press.

Najavits, L. M., Crits-Christoph, P. \& Dierberger, A. (2000). Clinicians' impact on the quality of substance use disorder treatment. Substance Use \& Misuse, 35(12-14), 2161-2190.

Narrow, W. E., Rae, D. S., Robins, L. N. \& Regier, D. A. (2002). Revised prevalence estimates of mental disorders in the United States: Using a clinical significance criterion to reconcile 2 surveys' estimates. Archives of General Psychiatry, 59, 115-124.

National Association of Social Workers. (approved 1996, revised 2008). Code of Ethics of the National Association of Social Workers. Retrieved from:

http://www.naswdc.org/pubs/code/code.asp

National Association of Social Workers, Alcohol, Tobacco, and Other Drugs Standards Work Group (2005). NASW standards for social work practice with clients with substance use disorders. Washington, DC: Author.

The National Center on Addiction and Substance Abuse at Columbia University (2009). Shoveling up II: The impact of substance abuse on federal, state and local budgets. New York: Author.

National Institute on Drug Abuse (1998). Drug use among racial/ethnic minorities. NIH Publication No. 98-3888. Rockville, MD: Author.

National Institute on Drug Abuse (2000). NIDA notes: Facts about drug abuse and Hepatitis C. Retrieved from http://archives.drugabuse.gov/nida_notes/nnvol15n1/tearoff.html.

Neighbors, H. W. \& Howard, C. S. (1987). Sex differences in professional help seeking among adult black Americans. American Journal of Community Psychology, 15(4), 403-417.

Norcross, J. C., Krebs, P. M., \& Prochaska, J. O. (2011). Stages of change. Journal of Clinical Psychology: In Session, 67(2), 143-154. doi: 10.1002/jclp.20758

Nyamathi, A., Longshore, D., Galaif, E. R. \& Leake, B. (2004). Motivation to stop substance use and psychological and environmental characteristics of homeless women. Addictive Behaviors, 29, 1839-1843. doi:10.1016/j.addbeh.2004.03.032

O'Callaghan, F. V., Chant, D. C., Callan, V. J., \& Baglioni, A. (1997). Models of alcohol use by young adults: An examination of various attitude- behavior theories. Journal of Studies on Alcohol, 58, 502-507. 
Ondersma, S. J., Svikis, D. S., \& Schuster, C. R. (2007). Computer-based brief intervention: A randomized trial with postpartum women. American Journal of Preventative Medicine, 32, 231-238.

Osborne, V. A. \& Benner, K. (2012). Utilizing screening, brief intervention, and referral to treatment: Teaching assessment of substance abuse. American Journal of Public Health, 102(7), e37-e38.

Pallant, J. (2007). SPSS survival manual: A step by step guide to data analysis using SPSS for Windows ( $3^{\text {rd }}$ ed.). New York: McGraw-Hill.

Palmer, R. S., Murphy, M. K., Piselli, A. \& Ball, S. A. (2009). Substance user treatment dropout from client and clinicial perspectives: A pilot study. Substance Use \& Misuse, 44, 1021-1038. doi: 10.1080/10826080802495237

Peer, K., Rennert, L., Lynch, K. G., Farrer, L., Gelernter, J. \& Kranzler, H. R. (2013). Prevalence of DSM-IV and DSM-5 alcohol, cocaine, opioid, and cannabis use disorders in a largely substance dependent sample. Drug and Alcohol Dependence, 127, 215-219. doi: 10.1016/j.drugalcdep.2012.07.009

Pemberton, M. R., Williams, J., Herman-Stahl, M., Calvin, S. L., Bradshaw, M. R., Bray, R. M. ... Mitchell, G. M. (2011). Evaluation of two web-based alcohol interventions in the US Military. Journal of Studies on Alcohol and Drugs, 72, 480-489.

Polcin, D. L. \& Weisner, C. (1999). Factors associated with coercion in entering treatment for alcohol problems. Drug and Alcohol Dependence, 54, 63-68.

Prochaska, J.O., Norcross, J.C., \& DiClemente, C.C. (1995). Changing for good. New York: Avon.

RachBeisel, J., Dixon, L. \& Gearon, J. (1999). Awareness of substance abuse problems among dually-diagnosed psychiatric inpatients. Journal of Psychoactive Drugs, 31(1), 53-57.

Rapp, L. A., Dulmus, C. N., Wodarski, J. S. \& Feit, M. D. (1999). Screening of substance abuse in public welfare and child protective service clients: A comparative study of rapid assessment instruments vs. the SASSI. Journal of Addictive Diseases, 18(2), 83-88. doi:10.1300/J069v18n02_08

Regier, D. A., Kaelber, C. T., Rae, D. S., Farmer, M. E., Knauper, B., Kessler, R. C. \& Norquist, G. S. (1998). Limitations of diagnostic criteria and assessment instruments for mental disorders: Implications for research and policy. Archives of General Psychiatry, 55, 109-115. 
Research Training Institute (2012). 2010 National Survey on Drug Use and Health public use file codebook. Rockville, MD: SAMHSA.

Restrepo, C. S., Carrillo, J. A., Martinez, S., Ojeda, P., Rivera, A. L. \& Hatta, A. (2007). Pulmonary complications from cocaine and cocaine-based substances: Imaging manifestations. RadioGraphics, 27, 941-956. doi: 10.1148/rg.274065144

Rockett, I. R. H., Putnam, S. L., Jia, H. \& Smith, G. S. (2003). Assessing substance abuse treatment need: A statewide hospital emergency department study. Annals of Emergency Medicine, 41(6), 802-813.

Rockett, I. R. H., Putnam, S. L., Jia, H., Chang, C. F. \& Smith, G. S. (2005). Unmet substance abuse treatment need, health services utilization, and cost: A population-based emergency department study. Annals of Emergency Medicine, 45(2), 118-127. doi: 10.1016/j.annemergmed.2004.08.003

Room, R. (2007). Taking account of cultural and societal influences on substance use diagnoses and criteria. Addiction, 101(Supplement 1), 31-39.

Rutherford, L. G. \& DeVaney, S. A. (2009). Utilizing the theory of planned behavior to understand convenience use of credit cards. Journal of Financial Counseling and Planning, 20(2), 48-63.

Ryan, R. M., Patrick, H., Deci, E. L. \& Williams, G. C. (2008). Facilitating health behavior change and its maintenance: Interventions based on Self-Determination Theory. European Health Psychology Society, 10(1), 2-5.

Ryan, R. M., Plant, R. W. \& O’Malley, S. (1995). Initial motivations for alcohol treatment: Relations with patient characteristics, treatment involvement, and dropout. Addictive Behaviors, 20(3), 279-297.

Rye, B. J., Fisher, W. A. \& Fisher, J. D. (2001). The theory of planned behavior and safer sex behaviors of gay men. AIDS and Behavior, 5(4), 307-317.

Salkind, N. J. (2003). Exploring research (5 ${ }^{\text {th }}$ ed.). Upper Saddle River, NJ: Prentice Hall.

Schafer, J. L. \& Graham, J. W. (2002). Missing data: Our view of the state of the art. Psychological Methods, 7(2), 147-177. doi: 10.1037//1082-989X.7.2.147

Schnittker, J. \& McLeod, J. D. (2005). The social psychology of health disparities. Annual Review of Sociology, 31, 75-103. doi: 10.1146/annuarev.soc.30.012703.110622

Schonfeld, L., King-Kallimanis, B. L., Duchene, D. M., Etheridge, R. L., Herrera, J. R., Barry, K. L. \& Lynn, N. (2010). Screening and brief intervention for substance 
misuse among older adults: The Florida BRITE Project. American Journal of Public Health, 100(1), 108-114. doi:10.2105/AJPH.2008.149534

Shapiro, D. L. \& Watson, A. (2000). Using the theory of planned behavior to induce problem solving in schools. Negotiation Journal, 16(2), 183-190.

Shepherd, C. B. \& Rickard, K. M. (2011). Drive for muscularity and help-seeking: The mediational role of gender role conflict, self-stigma, and attitudes. Psychology of Men \& Masculinity. Advance online publication. doi: 10.1037/a0025923

Smith, J. P., Tran, G. Q. \& Thompson, R. D. (2008). Can the Theory of Planned Behavior help explain men's psychological help-seeking? Evidence for a mediation effect and clinical implications. Psychology of Men \& Masculinity, 9(3), 179-192. doi: $10.1037 / \mathrm{a} 0012158$

Sobell, M. B. \& Sobell, L. C. (2005). Guided self-change model of treatment for substance use disorders. Journal of Cognitive Psychotherapy: An International Quarterly, 19(3), 199-210.

Sparks, P. \& Shepherd, R. (1992). Self-identity and the theory of planned behavior: Assessing the role of identification with "green consumerism." Social Psychology Quarterly, 55(4), 388-399.

Spitzer, R. L. (1998). Diagnosis and need for treatment are not the same. Archives of General Psychiatry, 55, 120.

Stein, M. D., Hagerty, C. E., Herman, D. S., Phipps, M. G., \& Anderson, B. J. (2008). A brief marijuana intervention for non-treatment-seeking young adult women. Journal of Substance Abuse Treatment, 34, 407-414. doi:10.1016/j.jsat.2007.07.004.

Stewart, D. (2009). Drug use and perceived treatment need among newly sentenced prisoners in England and Wales. Addiction, 104, 243-247. doi:10.1111/j.13600443.2008.02439.x

Stolzenberg, L., D’Alessio, S. J., Rivers, J. E. \& Newell, A. L. (2003). Measuring substance abuse treatment need among adults in Florida: A social indicators approach. Social Indicators Research, 61(1), 19-57.

Substance Abuse and Mental Health Services Administration (2013). SAMHSA's Working Definition of Recovery: 10 Guiding Principles. Retrieved from http://store.samhsa.gov/shin/content/PEP12-RECDEF/PEP12-RECDEF.pdf

Substance Abuse and Mental Health Services Administration (2012a). National Survey of Substance Abuse Treatment Services (N-SSATS): 2011. Data on Substance Abuse 
Treatment Facilities. BHSIS Series S-64, HHS Publication No. (SMA) 12-4730. Rockville, MD: Author.

Substance Abuse and Mental Health Services Administration (2012b). SAMHSA awards more than \$22 million for Screening, Brief Intervention, and Referral to Treatment Programs [News Release]. Retrieved from http://www.samhsa.gov/newsroom/advisories/1207254120.aspx

Substance Abuse and Mental Health Services Administration, Office of Applied Studies. (2010a). Treatment Episode Data Set (TEDS). 1998 - 2008. National Admissions to Substance Abuse Treatment Services, DASIS Series: S-50, HHS Publication No. (SMA) 09-4471, Rockville, MD: Author.

Substance Abuse and Mental Health Services Administration, Office of Applied Studies. (2010b). Results from the 2009 National Survey on Drug Use and Health: Volume I. Summary of National Findings (N SDUH Series H-38A, HHS Publication No. SMA 10-4586 Findings). Rockville, MD: Author.

Substance Abuse and Mental Health Services Administration, Office of Applied Studies. (2008). Treatment Episode Data Set (TEDS): 2005. Discharges from Substance Abuse Treatment Services, DASIS Series: S-41, DHHS Publication No. (SMA) 08-4314, Rockville, MD: Author.

Swendsen, J., Anthony, J. C., Conway, K. P., Degenhardt, L., Dierker, L., Glantz, M., ... Merikangas, K. R. (2008). Improving targets for the prevention of drug use disorders: Sociodemographic predictors of transitions across drug use stages in the National Comorbidity Survey Replication. Prevention Medicine, 47(6), 629634. doi:10.1016/j.ypmed.2008.09.009

Ta, V. M., Juon, H. S., Gielen, A. C., Steinwachs, D. \& Duggan, A. (2008). Disparities in use of mental health and substance abuse services by Asian and Native Hawaiin/Other Pacific Islander women. Journal of Behavioral Health Services \& Research, 35(1), 20-36.

Tabachnick, B. G. \& Fidell, L. S. (2007). Using multivariate statistics $\left(5^{\text {th }}\right.$ ed.). Boston, MA: Allyn and Bacon.

Tatarsky, A. (2003). Harm reduction psychotherapy: Extending the reach of traditional substance use treatment. Journal of Substance Abuse Treatment, 25, 249-256. doi: 10.1016/S0740-5472(03)00085-0

Tetrault, J. M., Desai, R. A., Becker, W. C., Fiellin, D. A., Concato, J. \& Sullivan, L. E. (2007). Gender and non-medical use of prescription opioids: Results from a national US survey. Addiction, 103, 258-268. doi: 10.1111/j.13600443.2007.02056.x 
Timko, C., Finney, J. W. \& Moos, R. H. (2005). The 8-year course of alcohol abuse: Gender differences in social context and coping. Alcoholism: Clinical and Experimental Research, 29(4), 612-621. doi: 10.1097/01.ALC.0000158832.07705.22

Timko, C., Moos, R. H., Finney, J. W. \& Connell, E. G. (2002). Gender differences in help-utilization and the 8-year course of alcohol abuse. Addiction, 97, 877-889.

Torrens, M., Gilchrist, G., Domingo-Salvany, A. \& the psyCoBarcelona Group. (2011). Psychiatric comorbidity in illicit drug users: Substance-induced versus independent disorders. Drug and Alcohol Dependence, 113, 147-156. doi:10.1016/j.drugalcdep.2010.07.013

Tucker, J. A. (1995). Predictors of help-seeking and the temporal relationship of help to recovery among treated and untreated recovered problem drinkers. Addiction, 90, 805-809.

Tucker, J. A. \& Simpson, C. A. (2011). The recovery spectrum: From self-change to seeking treatment. Alcohol Research \& Health, 33(4), 371-379.

Tucker, J. S., Wenzel, S. L., Golinelli, D., Zhou, A. \& Green, H. D. (2011). Predictors of substance abuse treatment need and receipt among homeless women. Journal of Substance Abuse Treatment, 40, 287-294. doi: 10.1016/j.jsat.2010.11.006

Ullman, J. B. (2001). Structural equation modeling. In B. G. Tabachnick \& L. S. Fidell (Eds.), Using multivariate statistics (pp.653-771). Boston, MA: Allyn and Bacon.

United States Department of Health and Human Services. Centers for Disease Control and Prevention. National Center for Health Statistics. (2011). Health, United States, 2010 with special feature on death and dying. Hyattsville, MD: Author.

United States Department of Health and Human Services. Substance Abuse and Mental Health Services Administration. Center for Behavioral Health Statistics and Quality. (2011). National Survey on Drug Use and Health, 2010. ICPSR32722v1. Ann Arbor, MI: Inter-university Consortium for Political and Social Research. doi:10.3886/ICPSR32722.v1

van Beljouw, I., Verhaak, P., Prins, M., Cuijpers, P., Penninx, B. \& Bensing, J. (2010). Reasons and determinants for not receiving treatment for common mental disorders. Psychiatric Services, 61, 250-257.

Vaillant, G. E. (1995). The natural history of alcoholism revisited. Cambridge, MA: Harvard University Press. 
Wakefield, J. C. (1992a). Disorder as harmful dysfunction: A conceptual critique of DSM-III-R's definition of mental disorder. Psychological Review, 99(2), 232-247.

Wakefield, J. C. (1992b). The concept of mental disorder: On the boundary between biological facts and social values. American Psychologist, 47(3), 373-388.

Walitzer, K. S. \& Dearing, R. L. (2006). Gender differences in alcohol and substance use relapse. Clinical Psychology Review, 26, 128-148. doi: 10.1016/j.cpr.2005.11.003

Wang, P. S., Berglund, P., Olfson, M., Pincus, H. A., Wells, K. B. \& Kessler, R. C. (2005a). Failure and delay in initial treatment contact after first onset of mental disorders in the National Comorbidity Survey Replication. Archives of General Psychiatry, 62, 603-613.

Wang, P. S., Lane, M., Olfson, M., Pincus, H. A., Wells, K. B. \& Kessler, R. C. (2005b). Twelve-month use of mental health services in the United States: Results from the National Comorbidity Survey Replication. Archives of General Psychiatry, 62, 629-640.

Warner, L. A., Kessler, R. C., Hughes, M., Anthony, J. C. \& Nelson, C. B. (1995). Prevalence and correlates of drug use and dependence in the United States. Archives of General Psychiatry, 52, 219-229.

Weisner, C. (1993). Toward an alcohol treatment entry model: A comparison of problem drinkers in the general population and in treatment. Alcoholism: Clinical and Experimental Research, 17(4), 746-752.

Weisner, C., Mertens, J., Tam, T. \& Moore, C. (2001). Factors affecting the initiation of substance abuse treatment in managed care. Addiction, 96, 705-716. doi: $10.1080 / 09652140020039071$

Weisner, C. \& Schmidt, L. A. (2001) Rethinking access to alcohol treatment. In M. Galater (Ed.), Recent Developments in Alcoholism, Volume 15: Services Research in the Era of Managed Care (pp. 107-136). New York: Kluwer Academic/Plenum Press.

Wild, T. C. (2006). Social control and coercion in addiction treatment: Towards evidence-based policy and practice. Addiction, 101, 40-49. doi:10.1111/j.13600443.2005.01268.x

Wild, T. C., Roberts, A. B. \& Cooper, E. L. (2002). Compulsory substance abuse treatment: Overview of recent findings and issues. European Addiction Research, 8, 84-93. 
Wild, T. C., Cunningham, J. A. \& Ryan, R. M. (2006). Social pressure, coercion, and client engagement at treatment entry: A self-determination theory perspective. Addictive Behaviors, 31, 1858-1872. doi:10.1016/j.addbeh.2006.01.002

Williamson, S., Gossop, M., Powis, B., Griffiths, P., Fountain, J. \& Strang, J. (1997). Adverse effects of stimulant drugs in a community sample of drug users. Drug and Alcohol Dependence, 44, 87-94.

Wilson, C. J., Deane, F. P., Marshall, K. L. \& Dalley, A. (2008). Reducing adolescents' perceived barriers to treatment and increasing help-seeking intentions: effects of classroom presentations by general practitioners. Journal of Youth and Adolescence, 37(10), 1257-1269.

Witkiewitz, K. \& Marlatt, G. A. (2007). Modeling the complexity of post-treatment drinking: It's a rocky road to relapse. Clinical Psychology Review, 27, 724-738. doi:10.1016/j.cpr.2007.01.002

World Health Organization (2007). Expert Committee on Problems Related to Alcohol Consumption, Second Report. Geneva, Switzerland: Author.

Wu, A. M. \& Tang, C. S. (2012). Problem gambling of Chinese college students: Application of the theory of planned behavior. Journal of Gambling Studies, 28(2), 315-324. doi: 10.1007/s10899-011-9250-4

Wu, L. T. \& Ringwalt, C. L. (2004). Alcohol dependence and use of treatment services among women in the community. American Journal of Psychiatry, 161(10), 1790-1797.

Wu, L. T., Ling, W., Burchett, B., Blazer, D. G., Yang, C., Pan, J. J., ... Woody, G. E. (2011). Use of item response theory and latent class analysis to link polysubstance use disorders with addiction severity, HIV risk, and quality of life among opioid-dependent patients in the Clinical Trials Network. Drug and Alcohol Dependence, 118, 186-193. doi:10.1016/j.drugalcdep.2011.03.018

Yu, J., Clark, L. P., Chandra, L., Dias, A., \& Lai, T.F. (2009). Reducing cultural barriers to substance abuse treatment among Asian Americans: A case study in New York City. Journal of Substance Abuse Treatment, 37, 398-406.

Zeldman, A., Ryan, R. M. \& Fiscella, K. (2004). Motivation, autonomy support, and entity beliefs: Their role in methadone maintenance treatment. Journal of Social and Clinical Psychology, 23(5), 675-69 
VITA

SHEILA P. VAKHARIA

2004

2004

B.A., Clinical and Social Psychology

University of Rochester

Rochester, New York

Fannie Bigelow Prize for Women's Leadership

University of Rochester

Rochester, New York

2006

M.S.W., Advanced Generalist

Binghamton University

Binghamton, New York

2006-2007

Social Worker

Addiction Center of Broome County

Binghamton, New York

2007-2010

Harm Reduction Social Worker

Housing Works

New York City, New York

2010-Present

Research Assistant

School of Social Work

Florida International University

Miami, Florida

\section{PUBLICATIONS AND PRESENTATIONS}

Macgowan, M. J. \& Vakharia, S. P. (expected 2013). Evidence-based practice:

Challenges and opportunities in the global context. In G. Palattiyil, D. Sidhva \& M. Chakrabarti (Eds.), Social Work in a Global Context: Issues and Challenges.

Macgowan, M. J. \& Vakharia, S. P. (2012). Teaching standards-based group work competencies to social work students: An empirical examination. Research on Social Work Practice, 22, 380-388. doi: 10.1177/1049731512442249

Wong, S. E. \& Vakharia, S. P. (2012). Teaching research and practice evaluation skills to graduate social work students. Research on Social Work Practice, 22,714-718. doi: $10.1177 / 1049731512451060$ 
Vakharia, S. P. \& Thomlison, B. (November 2012). Theoretical and practical issues in selecting substance use measures for practice evaluation. Paper presented at the Annual Meeting of the Council on Social Work Education, Washington, DC.

Vakharia, S. P. (November 2012). The effectiveness of psychosocial harm reduction interventions for adult substance using populations. Paper presented at Harm Reduction Coalition Annual Conference, Portland, OR.

Vakharia, S. P. (November 2012). Roundtable discussion: A working definition of recovery and harm reduction. Harm Reduction Coalition Annual Conference, Portland, OR.

Wong, S. E. \& Vakharia, S. P. (May 2012). Improving web browsing for community resources by clients with severe mental disorders. Paper presented at meeting of Association for Behavior Analysis International Convention, Seattle, WA.

Vakharia, S. P. (September 2011). Harm Reduction and Mental Health. Panel Discussion. North Carolina Harm Reduction Coalition Conference, Raleigh, NC.

Vakharia, S. P. (November 2010). Drug User Stigma. Symposium Panel Session at the Harm Reduction Coalition Annual Conference, Austin, TX. 